EVALUATION OF METHODOLOGY FOR DELINEATION OF PROTECTION ZONES AROUND PUBLIC-SUPPLY WELLS IN WEST-CENTRAL FLORIDA

By John Vecchioli, James D. Hunn, and Walter R. Aucott

U.S. GEOLOGICAL SURVEY

Water-Resources Investigations Report 88-4051

Prepared in cooperation with the

FLORIDA DEPARTMENT OF ENVIRONMENTAL REGULATION

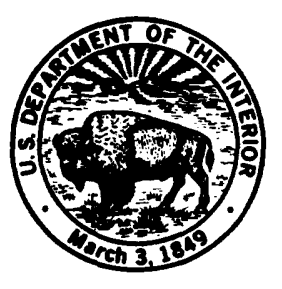

Tallahassee, Florida 


\section{DEPARTMENT OF THE INTERIOR \\ MANUEL LUJAN, JR., Secretary}

U.S. GEOLOGICAL SURVEY

Dallas L. Peck, Director

For additional information write to:

District Chief

U.S. Geological Survey

Suite 3015

227 North Bronough Street

Tallahassee, Florida 32301
Copies of this report can be purchased from:

U.S. Geological Survey

Books and Open-File Reports Section

Federal Center, Building 810

Box 25425

Denver, Colorado 80225 


\section{CONTENTS}

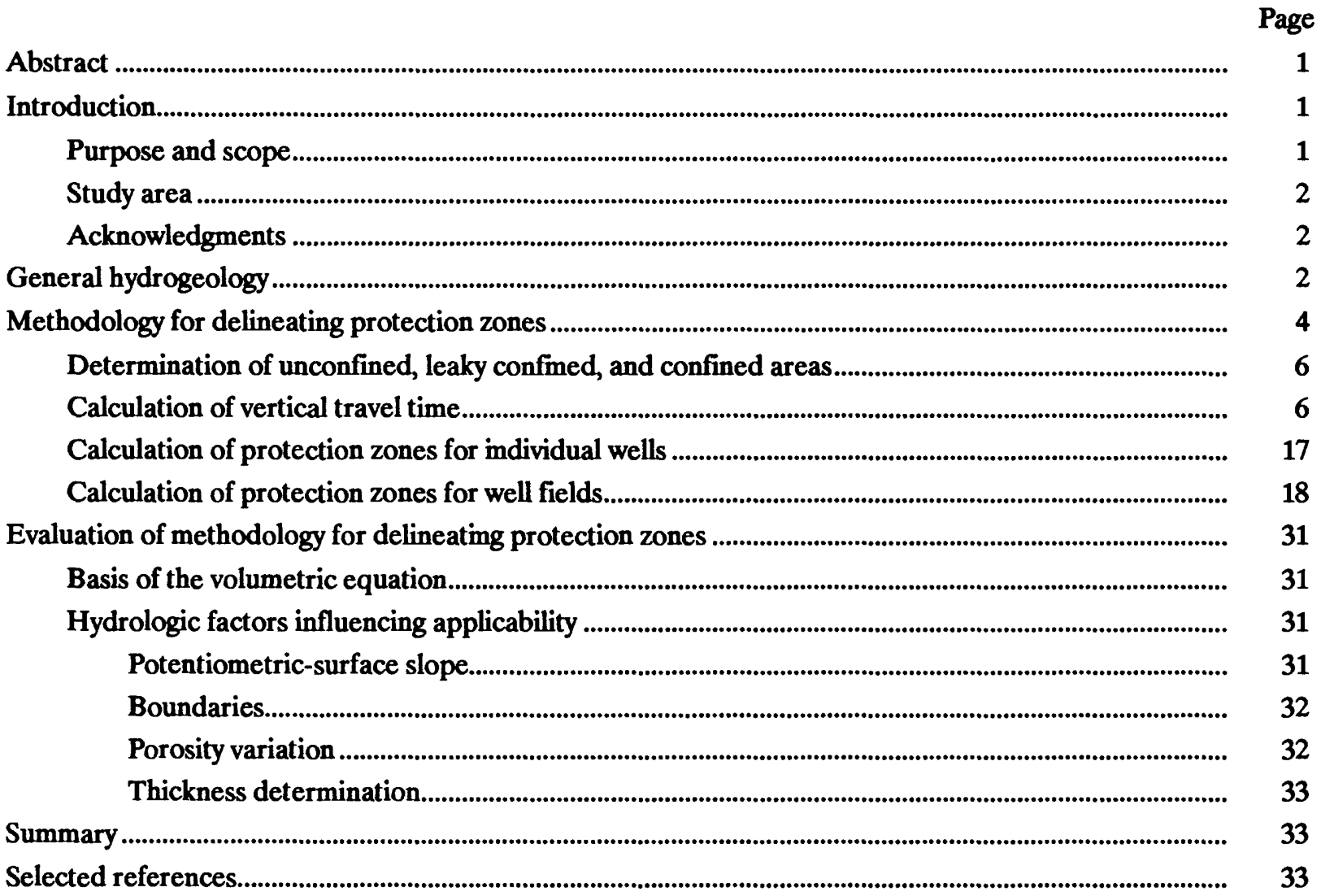

\section{ILLUSTRATIONS}

Plate

1-3. Maps of the protection zones for the:

1. Cypress Creek well field ...................................................................................................................... In pocket

2. Eldridge-Wilde well field ................................................................................................................. In pocket

3. Morris Bridge well field ................................................................................................................. In pocket

Figure

1-4. Maps of the:

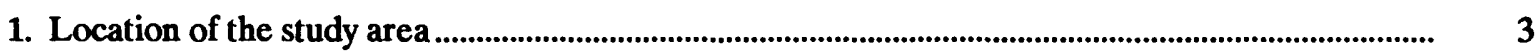

2. Potentiometric surface of the Upper Floridan aquifer, 1976 ......................................................... 5

3. Estimated potentiometric surface of the Upper Floridan aquifer prior to development ................ 7

4. Schematic of the procedure used in delineating protection zones......................................................

5-6. Maps of the location of:

5. Confined, leaky confined, and unconfined areas in the Floridan aquifer system in west-central Florida

6. Major public supply well fields in the five county area, west-central Florida ......................................

7-9. Maps of composite protection zones for:

7. Wells of the city of Crystal River, Citrus County

8. Wells of the Royal Palm Beach Colony, Inc., Hernando County...................................................... 29

9. Some wells of the West Coast Regional Water Supply Authority, Hillsborough County.................. 30 
Table

1. Hydrogeologic units in west-central Florida

2. Vertical travel-time calculations for well fields

3. Computation of vertical travel time and radius of well protection zones for wells in the leaky confined area

4. Computation of well protection zones in the unconfined area .................................................................. 19

5. Computation of well protection zones for well fields........................................................................... 21

6. Composite protection zones for well fields ........................................................................................................... 24

7. Composite protection zones for individual wells with overlapping zones of protection ............................ 25

8. Comparison of downgradient distance to stagnation point with radial distance calculated from volumetric equation.

\section{ABBREVIATIONS AND CONVERSION FACTORS}

For use of readers who prefer to use metric (International System) units, conversion factors for terms used in this report are listed below:

Multiply inch-pound unit

foot (ft)

foot per mile ( $\mathrm{ft} / \mathrm{mi})$

gallon per day (gal/d)

square foot $\left(\mathrm{ft}^{2}\right)$

foot squared per day $\left(\mathrm{ft}^{2} / \mathrm{d}\right)$

cubic foot per day $\left(\mathrm{ft}^{3} / \mathrm{d}\right)$

square mile $\left(\mathrm{mi}^{2}\right)$

gallon (gal)

million gallons per day (Mgal/d)
By

0.3048

0.1894

0.003785

0.09290

0.09290

0.02832

2.590

3.785

0.04381
To obtain metric unit

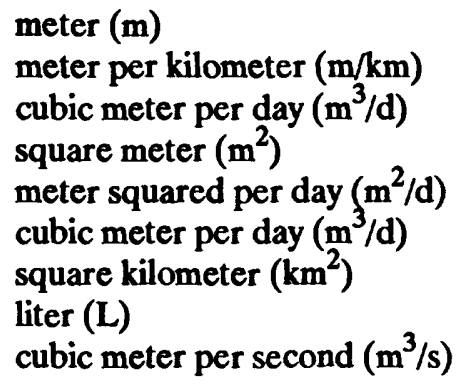

meter (m) meter per kilometer $(\mathrm{m} / \mathrm{km})$ cubic meter per day $\left(\mathrm{m}^{3} / \mathrm{d}\right)$ square meter $\left(\mathrm{m}^{2}\right)$ meter squared per day $\left(\mathrm{m}^{2} / \mathrm{d}\right)$ cubic meter per day $\left(\mathrm{m}^{3} / \mathrm{d}\right)$ square kilometer $\left(\mathbf{k m}^{2}\right)$ liter (L) cubic meter per second $\left(\mathrm{m}^{3} / \mathrm{s}\right)$

1 cubic foot per day $=7.48$ gallons per day

Sea level: In this report "sea level" refers to the National Geodetic Vertical Datum of 1929 (NGVD of 1929) - a geodetic datum derived from a general adjustment of the first-order level nets of both the United States and Canada, formerly called "Mean Sea Level of 1929." 


\title{
EVALUATION OF METHODOLOGY FOR DELINEATION OF PROTECTION ZONES AROUND PUBLIC-SUPPLY WELLS IN WEST-CENTRAL FLORIDA
}

\author{
By John Vecchioli, James D. Hunn, and Walter R. Aucott
}

\begin{abstract}
Public-supply wells in the west-central Florida area of Citrus, Hernando, Pasco, Hillsborough, and Pinellas Counties derive their supply solely from the Floridan aquifer system. Over most of that area, the Floridan is at or near land surface and vulnerable to contamination from surface sources. Recognizing the potential threat to this and other aquifers in the State, the Florida Department of Environmental Regulation recently promulgated regulations that provide for the designation and delineation of protection zones around public-supply wells that tap vulnerable aquifers, such as the Floridan in west-central Florida. This report evaluates the methodology used to define the protection zones for public-supply wells in west-central Florida in accordance with the methods detailed by the Florida Department of Environmental Regulation.
\end{abstract}

Protection zones were delineated for a total of 336 wells and 8 well fields that are permitted an average daily withdrawal of 100,000 gallons or more and that tap the Floridan aquifer system where it is unconfined or leaky confined. As specified in the regulations of the Florida Department of Regulation, leaky confined pertains to conditions such that the time for a particle of water to travel vertically from the water table to the top of the Floridan is 5 years or less. Protection zones were delineated by using a radial volumetric displacement model based on 5 years of permitted-rate withdrawal. Where zones of individual wells did not overlap, the radius of the circle encompassing the zone was tabulated. Where zones overlapped, such as for well fields, composite protection zones in shapes that varied according to the configuration of well arrays were delineated on maps, samples of which are included herein.

\section{INTRODUCTION}

Ground water is the source of drinking water for about 90 percent of Florida's residents. However, throughout much of Florida, aquifers that are tapped for public supply are vulnerable to contamination because they occur at relatively shallow depths and are overlain by materials that do not greatly impede downward movement of water. Recognizing this potential threat to the State's principal potable-water source, the Florida Department of Environmental Regulation
(FDER) has developed a "unique aquifer" category within the State's ground water classification scheme and has promulgated regulations that include provisions for defining and delineating "protection zones" around public-supply wells and well fields tapping unconfined or leaky confined aquifers (Florida Department of Environmental Regulation, 1987). These regulations prohibit or restrict certain activities having potential for contaminating the underlying ground water within a protection zone.

The FDER ground-water regulations (G-1 Rule) require that the State map protection zones around existing public-supply wells and well fields. This mapping activity was undertaken in 1987 as part of a cooperative study between the U.S. Geological Survey and the FDER in the west-central Florida area consisting of Citrus, Hernando, Pasco, Hillsborough, and Pinellas Counties. Similar mapping in other areas of Florida is planned by FDER.

\section{Purpose and Scope}

This report evaluates the methodology used to define protection zones for public supply wells in westcentral Florida in accordance with the methods given in the FDER regulations (1987). Hydrogeologic conditions at public-supply wells in west-central Florida are evaluated and calculations of the size of individual protection zones around each well or well field tapping unconfined or leaky confined parts of the Floridan aquifer system are presented. Public-supply wells in the west-central Florida area that are permitted an average daily withdrawal of 100,000 gallons or more are considered in this report.

Contained in the report is a map depicting the areas in which the Floridan aquifer system is under unconfined, leaky confined, or confined conditions. Other maps display the composite protection zone for selected well fields that contain wells having overlapping individual protection zones. Although composite protection zones were mapped for every instance of overlap, only selected samples are reproduced herein because of the perceived limited interest in each of the composite protection zone maps. Those not included are on file with FDER. Radii of circles encompassing 
the protection zone around individual wells with no overlapping protection zones are presented in tabular form along with pertinent location, construction, and withdrawal data for the wells.

The methodology used in delineating the protection zones is explained. Sources of hydrogeologic information used for making the needed calculations are described.

\section{Study Area}

The study area consists of five counties in westcentral Florida: Citrus, Hernando, Pasco, Hillsborough, and Pinellas (fig. 1). These counties range from sparsely populated, predominantly rural counties, such as Citrus in the north, to densely populated urban areas such as Pinellas County in the south.

\section{Acknowledgments}

The authors gratefully acknowledge the assistance provided by the Southwest Florida Water Management District (SWFWMD), and particularly David Moore, for providing the well data from well permit files. The City of St. Petersburg, the City of Tampa, the Pinellas County Water System, and the West Coast Regional Water Supply Authority also assisted in this investigation by providing maps of well fields.

\section{GENERAL HYDROGEOLOGY}

The five-county study area in west-central Florida is underlain by Eocene and younger sediments that, in parts of the area, comprise three principal hydrogeologic units: a surficial aquifer, the Floridan aquifer system, and an intermediate confining unit (table 1).

Table 1. - Hydrogeologic units in west-central Florida

[Modified from Ryder (1985), table 1]

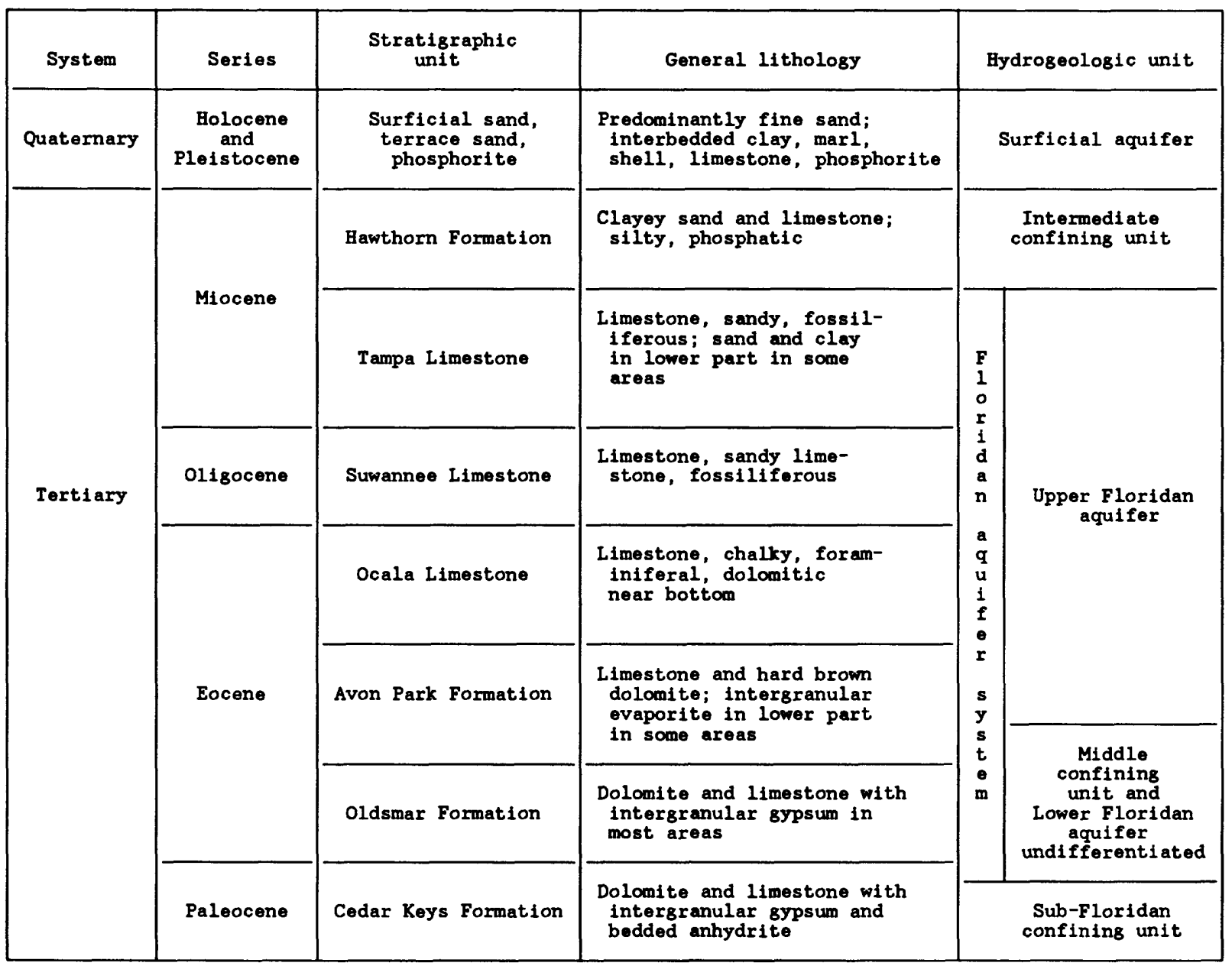




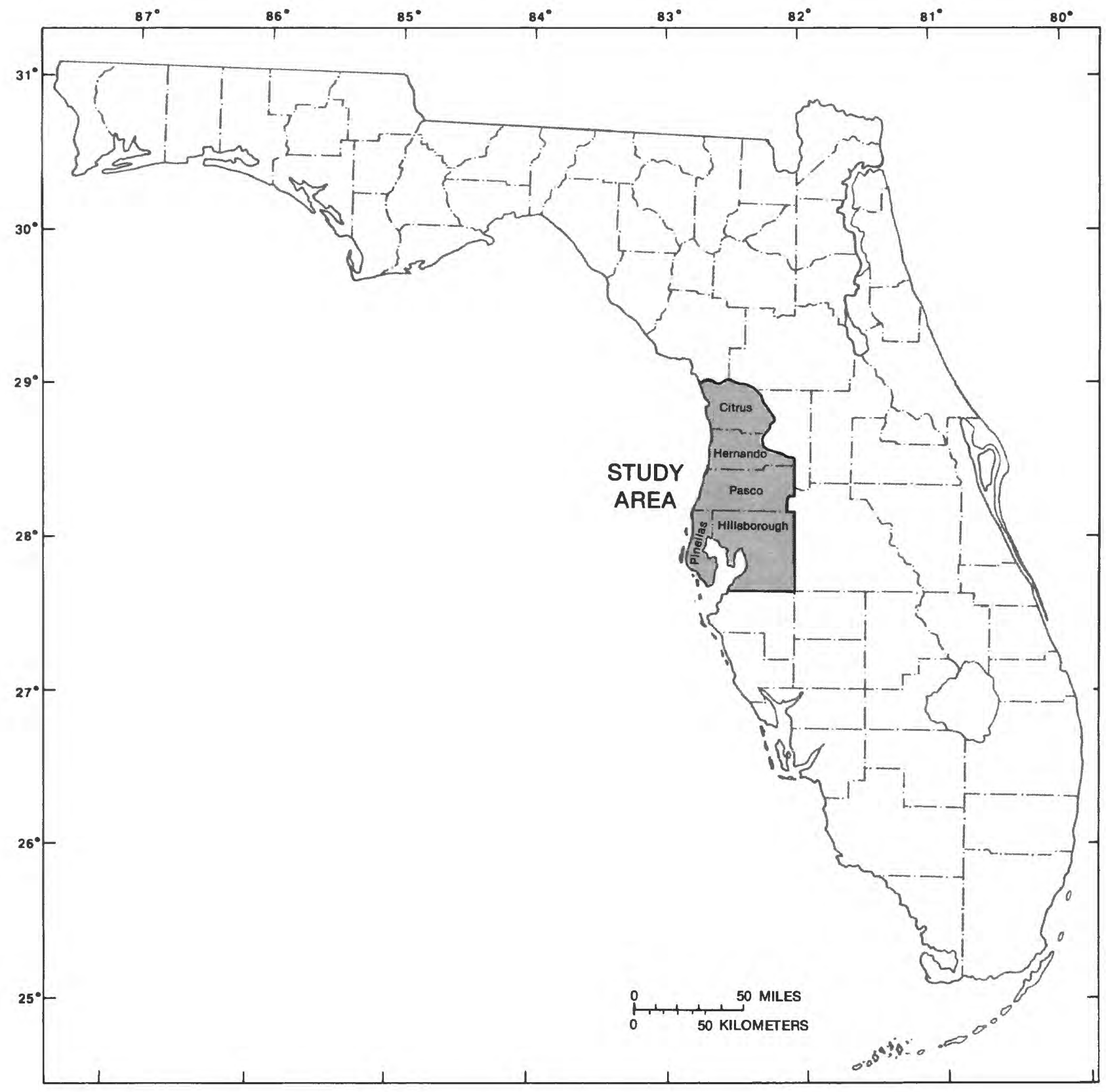

Figure 1.-Location of the study area.

The surficial aquifer is present in all but the northern part of the study area. The surficial deposits that make up the surficial aquifer generally consist of sand, clayey sand, shell, and shelly marl. The combined thickness of these deposits ranges from nearly zero in the north, where surficial deposits are thin and discontinuous over large areas, to greater than 50 feet in southern parts of the study area (Wolansky and others, 1979). Transmissivity of the surficial aquifer ranges from 205 to $1,800 \mathrm{ft}^{2} / \mathrm{d}$ and is much less than that of the Floridan aquifer system (Ryder, 1985; Wolansky and Corral, 1985). 
The intermediate confining unit consists of clayey sand and clay of the Hawthorn Formation. Its thickness and composition is highly variable in the study area. In the northern part, it is generally not present, whereas in the southern part, it occurs throughout most of the area and becomes progressively thicker and less permeable to the south. In the extreme southern part of the study area (southern Hillsborough County), the intermediate confining unit thickens and sand and limestone beds within it constitute aquifers. Where the unit is an important source of water, such as south of the study area in Manatee and Sarasota Counties, it is known as the intermediate aquifer system. Within the study area, these aquifers are very limited in extent and no public-supply wells tap them there; therefore, they are not considered in this report.

The principal water-producing unit in the study area is the Floridan aquifer system. All large-capacity municipal wells in the study area are open to the Floridan. The Floridan aquifer system is a vertically continuous sequence of Tertiary age carbonate rocks of high permeability (table 1) that are hydraulically connected in varying degrees, and whose permeability is several orders of magnitude greater than that of rocks that bound the system above and below. The Floridan aquifer system includes units of late Paleocene to early Miocene age, but within the study area, the formations of interest include the Avon Park Formation, Ocala Limestone, Suwannee Limestone, and the Tampa Limestone of Eocene to early Miocene age. Definition of the Floridan aquifer system and its relation to Florida's other regional hydrogeologic units is given by Southeastern Geological Society Ad Hoc Committee on Florida Hydrostratigraphic Unit Definition (1986). Miller (1986) presents a detailed geologic description of the Floridan, its component aquifers and confining units, and their relation to stratigraphic units throughout its geographic extent. The Floridan is referred to as an aquifer system because regionally it contains more than one distinct, highly permeable zone separated by a confining unit. In the study area, however, all municipal wells are open only to the upper part of the Floridan aquifer system referred to hereinafter as the Upper Floridan aquifer or the Floridan.

Transmissivities of the Upper Floridan aquifer in the study area range from 26,000 to more than 1 million $\mathrm{ft}^{2} / \mathrm{d}$ from reported aquifer tests and model analysis (Hutchinson, 1984; Ryder, 1985; Wolansky and Corral, 1985).

The flow system of the Upper Floridan aquifer is deducible from the potentiometric surface map in figure 2. Water flows from areas of high potential in central Florida (Polk and Lake Counties), and local potentiometric-surface highs, such as the one in Pasco County, westward toward the coast. Natural discharge is to rivers and streams, such as the Hillsborough and Withlacoochee Rivers, to springs which are prevalent in the coastal areas of Citrus and Hernando Counties, to the overlying surficial aquifer, and to the Gulf of Mexico and Tampa Bay. Hydraulic gradients average about $3 \mathrm{ft} / \mathrm{mi}$ or $0.0006 \mathrm{ft} / \mathrm{ft}$. In the northern half of the study area, the potentiometric surface of the Floridan aquifer system is little changed from predevelopment times (fig. 3) despite the large ground-water withdrawals from the area. In the southern half where the Floridan is confined, the potentiometric surface has declined over most of the area because of large ground-water withdrawals, mainly for agriculture and phosphate mining.

\section{METHODOLOGY FOR DELINEATING PROTECTION ZONES}

Protection zones were delineated according to criteria and methods in the FDER regulations (G-1 Rule), insofar as they were prescribed. The delineation of protection zones is a multistep process (fig. 4). The first step consists of determining whether the aquifer under consideration is unconfined, confined, or leaky confined. If unconfined, protection zones are required and the zone's size is calculated according to the method described later. If confined, protection zones beyond the inner zone of 200 feet radius that applies to all public-supply wells are not required and no further work is done. If leaky confined conditions are thought to occur, a calculation is made to determine average travel time for water to move vertically from the water table downward to the top of the aquifer. If the travel time is computed as 5 years or less, the aquifer is judged to be leaky confined (per FDER regulations) and a protection zone is required; its size is calculated similarly to that for the unconfined condition. If the travel time is greater than 5 years, the aquifer is considered confined and no protectionzone calculation is made.

The calculation of the size of protection zone required is performed first on an individual well basis and the area determined is simply circumscribed uniformly around the well. If the individual circumscribed zones overlap, the area of overlap is determined and distributed around the nonoverlapping parts of the individual zones. Where a number of wells are involved, such as in some of the large well fields, a composite protection zone is determined and distributed around the wells in the well field as described later. 


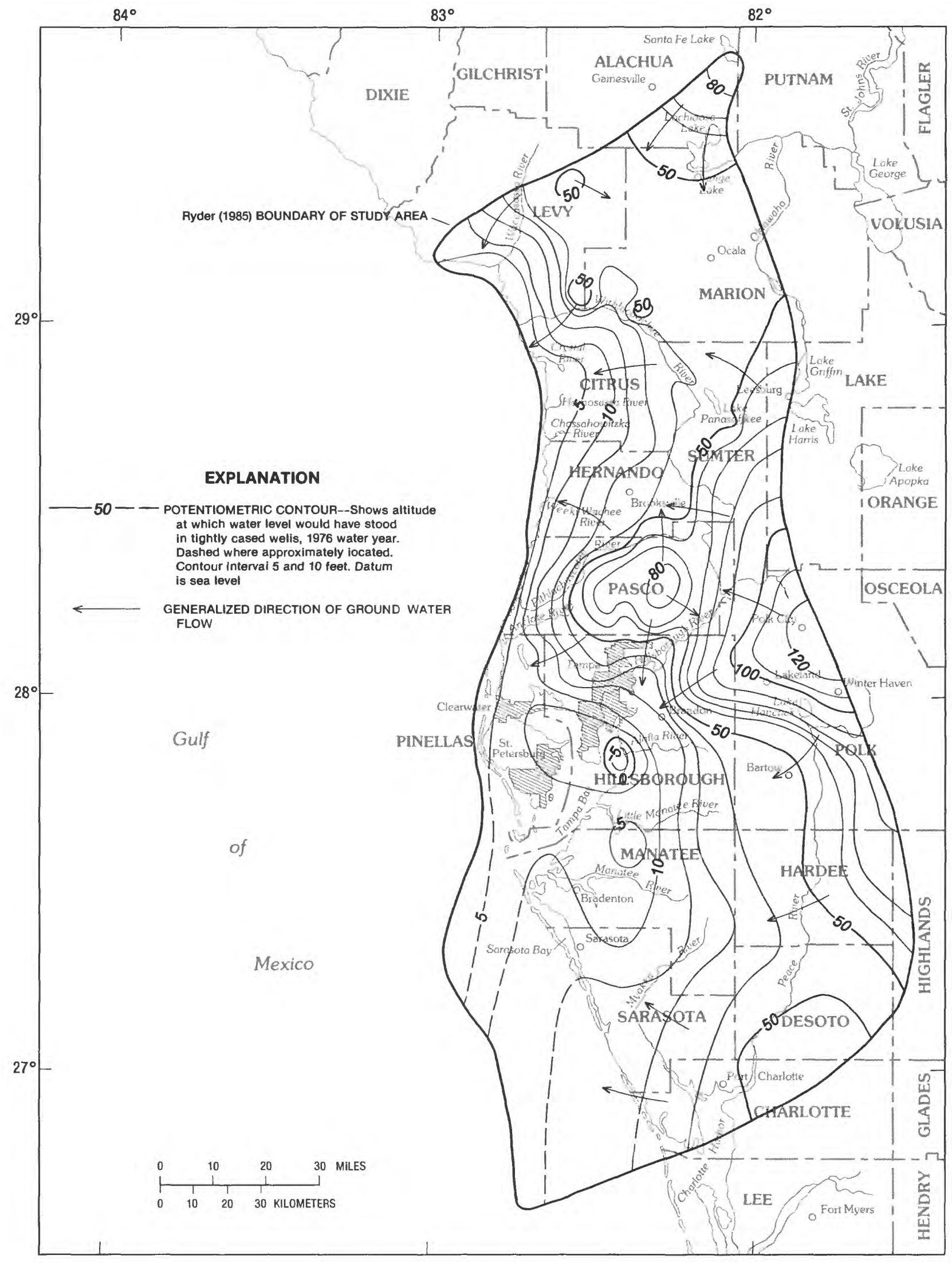

Figure 2. - Potentiometric surface of the Upper Floridan aquifer, 1976. (From Ryder, 1985, fig. 28.) 


\section{Determination of Unconfined, Leaky Confined, and Confined Areas}

A number of sources of information were used to determine where the Upper Floridan aquifer is unconfined, leaky confined, or confined (Buono and Rutledge, 1979; Buono and others, 1979; Ryder, 1985; Miller, 1986; and an unpublished map dated March 1985 prepared by the Southwest Florida Water Management District). Extent of the areas where these conditions occur in the west-central Florida study area is shown in figure 5. The unconfined/leaky confined boundary was determined using a combination of the references noted above. The leaky confined/confined boundary was generalized from the line where leakance of the confining unit overlying the Upper Floridan was less than $1 \times 10^{-4} \mathrm{ft} / \mathrm{d} / \mathrm{ft}$, as shown by Ryder (1985, p. 16). Leakance is the vertical hydraulic conductivity of the confining bed divided by its thickness and can be expressed as $\mathrm{K}_{\mathrm{z}} / \ell$. A leakance value of $1 \times 10^{-4} \mathrm{ft} / \mathrm{d} / \mathrm{ft}$, coupled with typical vertical head gradients prevalent in the confined area, results in vertical travel time calculations of much greater than 5 years. Therefore, it was considered a reasonable threshold value for bounding the area where the degree of confinement was such as to preclude the need to make site by site calculations of vertical travel time.

\section{Calculation of Vertical Travel Time}

The average vertical travel time for a particle of water to travel from the water table to the top of the Upper Floridan aquifer was computed for all wells or well fields in the area mapped as leaky confined. If the travel time at a particular well, or anywhere within a designated well field, was less than or equal to 5 years, the confining unit was considered to be sufficiently leaky to require that a protection zone be calculated. If the travel time was greater than 5 years throughout the well field, the confining unit was not considered to be leaky, as defined in the FDER regulation, and no protection zone was calculated.

Vertical travel time was calculated using the formulae specified in the FDER regulation (Florida Department of Environmental Regulation, 1987) which when combined yield:

$$
t_{v}=\frac{n \ell^{2}}{\mathrm{~K}_{\mathrm{z}} \Delta \mathrm{h}},
$$

derived from Darcy's law, where

$t_{v}=$ vertical travel time between water table and top of Upper Floridan aquifer; $\mathbf{n}=$ equivalent effective porosity of deposits between water table and top of Upper Floridan aquifer $=0.2$, an assumed value believed to be representative of the vertical section overlying the Upper Floridan aquifer;

$\ell=$ distance between water table and top of Upper Floridan aquifer;

$\mathrm{K}_{\mathrm{z}}$ = equivalent hydraulic conductivity of material between the water table and the top of the Upper Floridan aquifer; and

$\Delta \mathrm{h}=$ head difference between the potentiometric surface of the Upper Floridan aquifer and the water table.

Data used for the vertical travel-time calculations were derived from a number of sources. Data on the top of the Floridan were derived using Stewart (1968), Buono and Rutledge (1979), and well logs. The equivalent vertical hydraulic conductivity, $\mathrm{K}_{\mathrm{z}}$, is calculated by taking into account the individual vertical hydraulic conductivity and thickness of each layer of material between the water table and the top of the Upper Floridan aquifer. Mathematically, this can be expressed as (Freeze and Cherry, 1979, p. 34):

$$
K_{z}=\frac{d}{\sum_{i=1}^{n} d_{1} / K_{i}}
$$

where

$\mathrm{d}_{\mathrm{i}}$ and $\mathrm{K}_{\mathrm{i}}=$ the respective thickness and vertical hydraulic conductivity of individual layers, and

$$
\mathrm{d}=\text { the total thickness. }
$$

However, it can be shown that in the case of two layers-surficial aquifer underlain by a confining unit - the leakance of the two layers, which equals the equivalent vertical hydraulic conductivity divided by the surficial aquifer-confining unit thickness, approximates the leakance of the confining unit, which equals the vertical hydraulic conductivity of the confining unit divided by its thickness, where the confining unit conductivity is much smaller than the vertical hydraulic conductivity of the surficial aquifer, and the thickness of the surficial aquifer is not significantly greater than that of the confining unit. Therefore, for the surficial aquifer and confining unit materials extant in the study area, primarily sand and clay, respectively, it is reasonable to estimate their equivalent vertical hydraulic conductivity and thickness by considering only the hydraulic conductivity and thickness of the confining unit. 


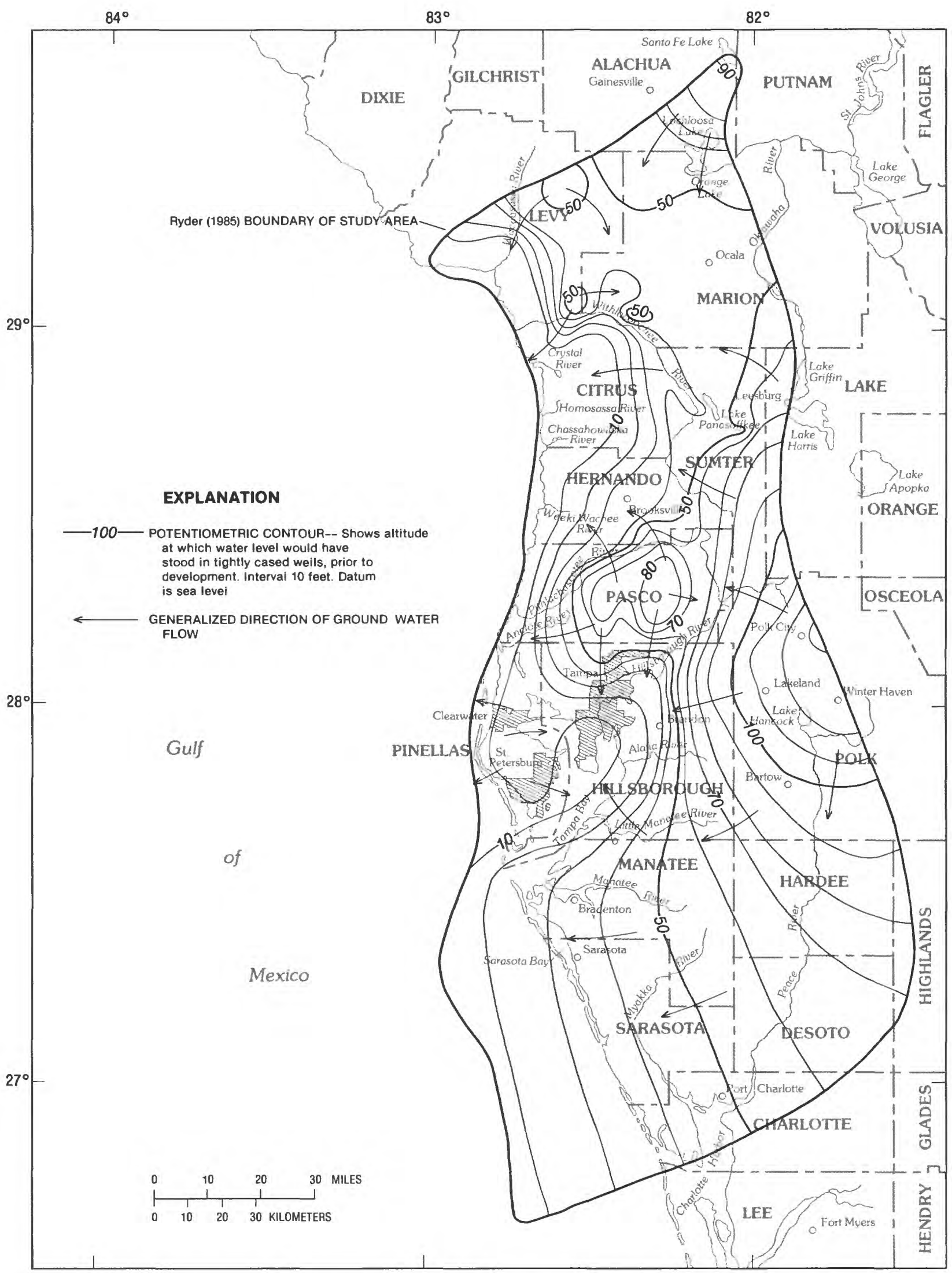

Figure 3. - Estimated potentiometric surface of the Upper Floridan aquifer prior to development (modified from Johnston and others, 1980). (From Ryder, 1985, fig. 17.) 


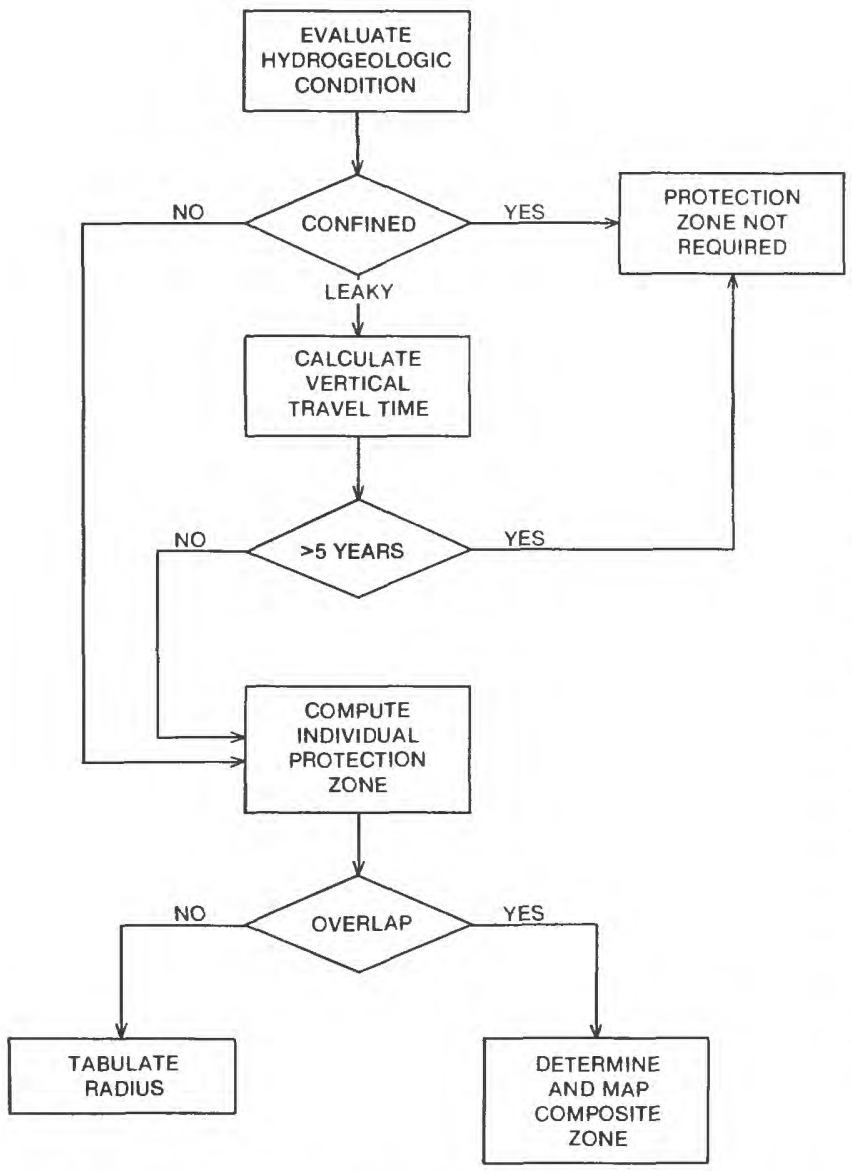

Figure 4. - Procedure used in delineating protection zones.
Data utilized for computing vertical travel times for the well fields were derived from a number of sources. Floridan heads and water-table altitudes were derived from Ryder and Mills (1977), Yobbi and others (1980), Yobbi and Woodham (1981), and Barr (1982), for times when the well fields were pumping near their average permitted withdrawal rates. No water-level maps were available for the Cross Bar Ranch and Starkey well fields for a period when they were pumping near their average permitted withdrawal rates. A model-simulated head difference from Hutchinson (1985) was used at the Cross Bar Ranch well field. Head difference was calculated for Starkey using analytical methods (Jacob, 1945; Lohman, 1972) combined with May 1982 water levels. The equivalent hydraulic conductivity was determined from model analyses of Hutchinson (1984) and Ryder (1985) and from aquifer tests for which confining bed characteristics were calculated (Wolansky and Corral, 1985). Data from wells in the leakiest part of the well field were used for the vertical calculation for each well field. The rationale was that if any well in a dense grouping of wells, such as in a well field, needed a protection zone, then all wells in that well field should be protected.

The location of the major well fields in the study area is shown in figure 6. The data utilized and the results of the vertical travel-time calculations for the 11 well fields located in the leaky confined part of the study area are listed in table 2. Eight of the eleven well

Table 2.-Vertical travel-time calculations for well fields

$[g a l / d=$ gallons per day, $f t / d / f t=$ feet per day per foot $]$

\begin{tabular}{|c|c|c|c|c|c|c|c|c|c|c|c|}
\hline \multirow[b]{2}{*}{$\begin{array}{l}\text { Well } \\
\text { field } \\
\text { name }\end{array}$} & \multirow{2}{*}{$\begin{array}{l}\text { Consump- } \\
\text { tive use } \\
\text { permit } \\
\text { number }\end{array}$} & \multirow{2}{*}{$\begin{array}{c}\text { Permitted } \\
\text { average } \\
\text { pumping } \\
\text { rate } \\
(\mathrm{gal} / \mathrm{d}) \\
\end{array}$} & \multicolumn{2}{|c|}{ Time of calculation } & \multicolumn{2}{|c|}{ Altitude } & \multirow{2}{*}{$\begin{array}{l}\text { Verti- } \\
\text { cal } \\
\text { head } \\
\text { differ- } \\
\text { ence } \\
\text { (feet) }\end{array}$} & \multirow{2}{*}{$\begin{array}{l}\text { Altitude } \\
\text { top of } \\
\text { Floridan } \\
\text { aquifer } \\
\text { system } \\
\text { (feet) }\end{array}$} & \multirow{2}{*}{$\begin{array}{l}\text { Vert1- } \\
\text { cal } \\
\text { dis- } \\
\text { tance } \\
\text { (feet) } 1\end{array}$} & \multirow{2}{*}{$\begin{array}{l}\text { Leakance } \\
\text { (ft/d/ft) }\end{array}$} & \multirow{2}{*}{$\begin{array}{c}\text { Vert1- } \\
\text { cal } \\
\text { travel } \\
\text { time } \\
\text { (days) }\end{array}$} \\
\hline & & & Date & $\begin{array}{l}\text { Pumping } \\
\text { rate } \\
\text { (gal/d) }\end{array}$ & $\begin{array}{l}\text { Water } \\
\text { table } \\
\text { (feet) }\end{array}$ & $\begin{array}{l}\text { Potentio- } \\
\text { metric } \\
\text { surface } \\
\text { (feet) }\end{array}$ & & & & & \\
\hline $\begin{array}{l}\text { South } \\
\text { Pasco }\end{array}$ & 0364701 & $16,900,000$ & May 1981 & $17,300,000$ & 55 & 37 & 18 & 5 & 50 & 0.00036 & 1,543 \\
\hline $\begin{array}{l}\text { Cypress } \\
\text { Creek }\end{array}$ & 0365002 & $30,000,000$ & May 1982 & $30,000,000$ & 63 & 49 & 14 & 40 & 23 & .0003 & 1,095 \\
\hline $\begin{array}{l}\text { Cross } \\
\text { Bar }\end{array}$ & 0429001 & $30,000,000$ & Simulated & $30,000,000$ & 50 & 45 & 5 & 30 & 20 & .0009 & 889 \\
\hline $\begin{array}{l}\text { Starkey } \\
\text { Eldridge- } \\
\text { Wilde }\end{array}$ & $\begin{array}{l}0444602 \\
0267301\end{array}$ & $\begin{array}{r}8,000,000 \\
35,244,000\end{array}$ & $\begin{array}{l}\text { Calculated } \\
\text { May } 1980\end{array}$ & $\frac{(2)}{27,400,000}$ & $\begin{array}{l}20 \\
22\end{array}$ & $\begin{array}{l}14 \\
13\end{array}$ & $\begin{array}{l}6 \\
9\end{array}$ & $\begin{array}{l}-10 \\
-10\end{array}$ & $\begin{array}{l}32 \\
32\end{array}$ & $\begin{array}{l}.0006 \\
.00054\end{array}$ & $\begin{array}{l}1,778 \\
1,317\end{array}$ \\
\hline $\begin{array}{c}\text { East Lake } \\
\text { Road }\end{array}$ & 0439101 & $3,000,000$ & May 1977 & $4,300,000$ & 10 & 3 & 7 & -25 & 35 & .00054 & 1,852 \\
\hline $\begin{array}{l}\text { Section } \\
21\end{array}$ & 0000301 & $13,000,000$ & May 1982 & $11,100,000$ & 48 & 34 & 14 & 15 & 33 & .0003 & 1,571 \\
\hline $\begin{array}{l}\text { Cosme- } \\
\text { Odessa }\end{array}$ & 0000401 & $13,000,000$ & May 1981 & $10,400,000$ & 42 & 14 & 28 & -10 & 52 & .0003 & 1,238 \\
\hline $\begin{array}{l}\text { Morris } \\
\text { Bridge }\end{array}$ & 0418000 & $15,500,000$ & May 1981 & $15,000,000$ & 32 & 28 & 4 & 20 & 12 & .0008 & 750 \\
\hline $\begin{array}{l}\text { Sun City } \\
\text { Riverview }\end{array}$ & $\begin{array}{l}0435201 \\
0435201\end{array}$ & $\begin{array}{l}3,600,000 \\
2,100,000\end{array}$ & $\begin{array}{ll}\text { May } & 1982 \\
\text { May } & 1980\end{array}$ & $\begin{array}{l}2,300,000 \\
1,900,000\end{array}$ & $\begin{array}{l}53 \\
56\end{array}$ & $\begin{array}{r}-5 \\
2\end{array}$ & $\begin{array}{l}58 \\
54\end{array}$ & $\begin{array}{l}-90 \\
-50\end{array}$ & $\begin{array}{l}143 \\
106\end{array}$ & $\begin{array}{l}.0000098 \\
.0000098\end{array}$ & $\begin{array}{l}50,000 \\
40,000\end{array}$ \\
\hline
\end{tabular}

1Water table to top of Floridan.

${ }^{2}$ Calculated based on individual well permit rates. 


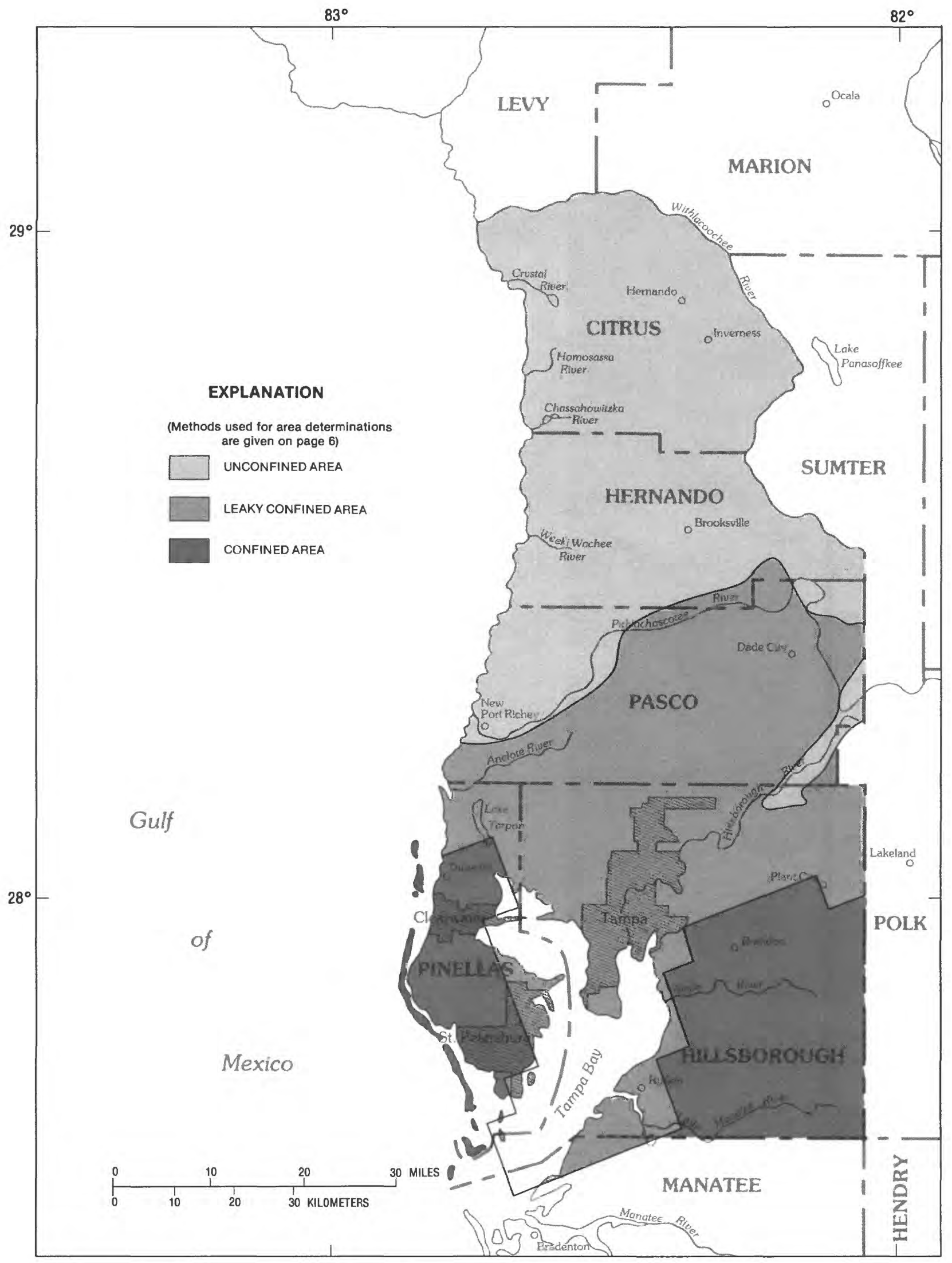

Figure 5. - Confined, leaky confined, and unconfined areas in the Floridan aquifer system in west-central Florida. 


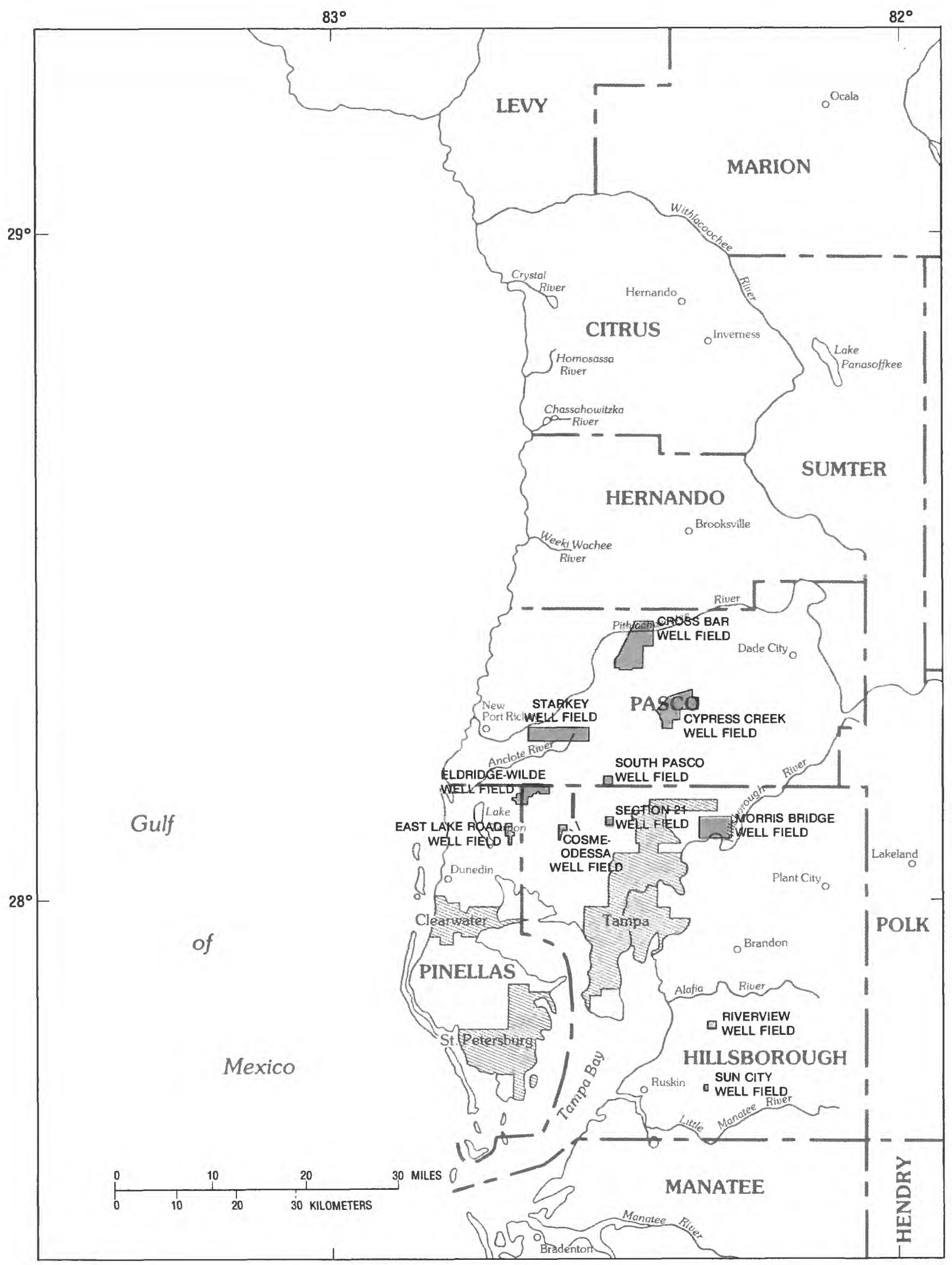

Figure 6. - Major public supply well fields in the five county area, west-central Florida. 
fields have computed vertical travel times of less than 5 years (1,825 days) and thus require protection zones. These include the Cross Bar Ranch, Cypress Creek, South Pasco, Eldridge-Wilde, Cosme-Odessa, Section 21, Morris Bridge, and Starkey well fields. These well fields are predominantly located in the northern part of the leaky confined area where the confining unit overlying the Floridan is the leakiest. Three of the eleven well fields, East Lake Road, Sun City, and Riverview, have computed vertical travel times of greater than 5 years and thus do not require protection zones.

The data utilized and the results of the vertical travel-time calculations for individual wells are listed in table 3. Floridan heads were derived from Barr (1982) and Barr and Schiner (1982). Water-table altitudes were derived from Barr (1982) and estimated from topographic maps outside the area Barr mapped. Because of the wide contour spacing and topographic relief in some areas of Barr's map, all interpolated water levels were checked against land surface and water features on U.S. Geological Survey 7-1/2-minute topographic maps and adjusted where necessary. The leakance of the confining unit overlying the Floridan, derived from Hutchinson (1984) and Ryder (1985), was substituted for $\mathrm{K}_{2} / \ell$ in equation (1), as explained previously.

Table 3.-Computation of vertical travel time and radius of well protection zones for wells in the leaky confined area

Sources and explanation of data for calculations:

We11 - Well number from Southwest Florida Water Management District (SWFWM) files. Blank where not available. Owner - From SWFWMD permitting files, February 2, 1987.

Cons. use permit - Consumptive use permit number. From SWFWM permitting files, February 2 , 1987.

Well name - From SWFWMD files.

County - From SWFWD permitting files, February 2, 1987.

Lat, Long, Sec. Twn, and Rng - Latitude, longitude, section, township, and range. From SwFwM permitting files, February 2,1987 .

Av. - - Permitted average pumping rate, in gallons per day, for public-supply use. From SWFWM permitting files, February 2,1987 .

Well depth, in feet - From SWFWMD files Blank where not available.

Dia - Well' diameter, in inches. From SWFWD permitting files, February $2,1987$.

Lsd - Altitude of land surface, in feet, from U.S. Geological Survey 7t-minute topographic maps at latitude and Iongitude given.

F1 - Altitude of top of Upper Floridan aquifer, in feet (Buono and Rutledge, 1979 ).

m - Thickness of Upper Floridan aquifer at well site (Miller, 1982).

I - Transmissivity, in feet squared per day, of Upper Floridan aquifer at well site (Hutchinson, 1984 ). Values of transmissivity outside Hutchinson's model area are from Ryder (1985).

$\underline{h}$ - Thickness of Upper Floridan aquifer, in feet, penetrated by the well (well depth-[Lsd-F1]).

WT - Altitude of the water table, in feet above sea level, at the well site, May 1982 (Barr, 1982 ). Because of the topographic relief and wide spacing of contours in some areas, interpolated water-table altitudes were checked against topographic maps and adjusted where necessary. Water-table altitudes outside the map area were estimated from USGS $7 \nmid$-minute topographic maps.

Pot - Altitude of the potentiometric surface of the Upper Floridan aquifer, in feet above sea level, at the well site, May 1982 (Barr, 1982; Barr and Schiner, 1982)

s - Drawdown of the potentiometric surface, in feet, after one day pumping at the rate shown under "Av.Q", in gallons per day, calculated by the modified nonequilibrium formula (Cooper and Jacob, 1946, cited in Lohman, 1972 , p. 19 ):

$$
s=\frac{(2.30)(\mathrm{Av} . \mathrm{Q})}{(7.48)(4)(\pi) \mathrm{T}} \quad \log _{10} \frac{(2.25)(\mathrm{T})(1)}{(\mathrm{Dia} / 24)^{2}(0.001)}
$$

$\underline{s(K)}$ - Drawdown, as above, corrected for partial penetration ( $h / m$ ) by the Kozeny (1933) formula, modified from Jacob (1945):

$$
s(K)=s /\left[h / m\left(1+7\left[\frac{D i a / 24}{2 h}\right] \cos \frac{\pi h / m}{2}\right)\right] \text {. }
$$

The argument for cosine is in radians. Tabulated values of $s(K)$ are truncated to integers.

$\Delta h$ - Difference in water-level altitude between the water table (WT) and the potentiometric surface (pot) minus drawdown $(s(K)) . \Delta h=W T-[\operatorname{Pot}-s(K)]$, in feet.

$\ell$ - Vertical distance, water table to top of Upper Floridan aquifer (WT-F1).

Leak - Leakance of the confining unit overlying the Upper Floridan aquifer, (Hutchinson, 1984), in feet per day per foot ( $\mathrm{ft} / \mathrm{d} / \mathrm{ft}$ ), or, in the case of two confining units, the equivalent leakance of both units. Values of leakance outside Hutchinson's model area are from Ryder (1985). Leakance equals vertical hydraulic conductivity divided by thickness.

$t_{v}$ - Calculated vertical travel time, in days, $=\frac{0.2(l)}{(1 e a k)(\Delta h+5)}$. The value of $\Delta h$ was increased by 5 feet, as explained on page 16, because of the approximate nature of the water table and potentiometric surface maps. ">1,825 days" means that calculated vertical travel time at the well site is 8 reater than 5 years, even if a corrected drawdown is applied for only 5 percent penetration of the Floridan.

$\underline{\underline{r}}$ - Radius of protection zone of the well $=\left[\frac{1825 \mathrm{Q}}{7.48 \pi \mathrm{hn}}\right]^{\frac{1}{2}}$, rounded to the nearest 10 feet $\mathrm{n}=0.05$ for the Upper Floridan aquifer. No entry indicates a vertical travel time greater than 5 years, hence no protection zone is calculated. "**" means that a protection zone is needed even if the well is fully penetrating, but the radius cannot be calculated because of missing well depth data. "*" means that the presence or absence of a protection zone depends on the partial penetration correction, which cannot be calculated because of missing well depth data. 
Table 3. - Computation of vertical travel time and radius of well protection zones for wells in the leaky confined area-Continued

\begin{tabular}{|c|c|c|c|c|c|c|c|c|c|c|c|}
\hline Well & Omer & $\begin{array}{c}\text { Cons. } \\
\text { use } \\
\text { permit }\end{array}$ & $\begin{array}{l}\text { Well } \\
\text { name }\end{array}$ & Lat. & Long. & Sec. & $\begin{array}{l}\text { Twn.; } \\
\text { (S.) }\end{array}$ & $\begin{array}{l}\text { Rng ; } \\
\text { (E.) }\end{array}$ & $\begin{array}{c}\text { Av.Q } \\
(\mathrm{gal} / \mathrm{d})\end{array}$ & $\begin{array}{r}\text { Well } \\
\text { depth } \\
\text { (ft) }\end{array}$ & $\begin{array}{l}\text { Dia } \\
\text { (in) }\end{array}$ \\
\hline \multicolumn{12}{|c|}{ Pasco County } \\
\hline $\begin{array}{l}00032 \\
00033 \\
00115 \\
00120 \\
00122 \\
00125 \\
00126 \\
00127 \\
00128 \\
00221\end{array}$ & $\begin{array}{r}\text { WATER \& SEWER DISTRICT A } \\
\text { WATER \& SEWER DISTRICT A } \\
\text { LYKES PASCO PACKING CO } \\
\text { BOLIDAY GARDENS UTILITIES INC. } \\
\text { CRESTRIDGE GARDENS UTIL CORP } \\
\text { CRESTRIDGE GARDENS UTIL CORP } \\
\text { CRESTRIDGE GARDENS UTIL CORP } \\
\text { CRITERION CORPORATION } \\
\text { CRITERION CORPORATION } \\
\text { DADE CITY FLORIDA, CITY OF }\end{array}$ & $\begin{array}{l}0002502 \\
0002502 \\
0045101 \\
0054002 \\
0054302 \\
0054302 \\
0054302 \\
0059001 \\
0059001 \\
0163101\end{array}$ & $\begin{array}{r}\text { C } \\
\text { D } \\
\text { MP2 } \\
1 \\
1 \\
4 \\
5 \\
1 \\
2 \\
1\end{array}$ & $\begin{array}{l}281148 \\
281143 \\
282230 \\
281129 \\
281034 \\
281045 \\
281052 \\
281026 \\
281110 \\
282148\end{array}$ & $\begin{array}{l}822629 \\
822629 \\
821124 \\
824340 \\
824405 \\
824358 \\
824357 \\
822505 \\
822537 \\
821130\end{array}$ & $\begin{array}{l}30 \\
30 \\
27 \\
29 \\
31 \\
31 \\
31 \\
33 \\
29 \\
27\end{array}$ & $\begin{array}{l}26 \\
26 \\
24 \\
26 \\
26 \\
26 \\
26 \\
26 \\
26 \\
24\end{array}$ & $\begin{array}{l}19 \\
19 \\
21 \\
16 \\
16 \\
16 \\
16 \\
19 \\
19 \\
21\end{array}$ & $\begin{array}{r}112,000 \\
112,000 \\
2,000,000 \\
128,000 \\
110,000 \\
130,000 \\
110,000 \\
131,000 \\
131,000 \\
500,000\end{array}$ & $\begin{array}{r}665 \\
620 \\
461 \\
85 \\
100 \\
170 \\
160 \\
575 \\
560 \\
400\end{array}$ & $\begin{array}{r}12 \\
8 \\
10 \\
8 \\
8 \\
12 \\
12 \\
12 \\
12 \\
8\end{array}$ \\
\hline $\begin{array}{l}00222 \\
00223 \\
00224 \\
00256 \\
00257 \\
00258 \\
00259\end{array}$ & $\begin{array}{r}\text { DADE CITY FLORIDA, CITY OF } \\
\text { DADE CITY FLORIDA, CITY OF } \\
\text { DADE CITY FLORIDA, CITY OF } \\
\text { BETMAR UTILITIES } \\
\text { BETMAR UTILITIES } \\
\text { BETMAR UTILITIES } \\
\text { BETMAR UTILITIES } \\
\text { ZARING CORPORATION } \\
\text { ZARING CORPORATION } \\
\text { LINDRICK SERVICE CORPORATION }\end{array}$ & $\begin{array}{l}0163101 \\
0163101 \\
0163101 \\
0203001 \\
0203001 \\
0203001 \\
0203001 \\
0285602 \\
0285602 \\
0297801\end{array}$ & $\begin{array}{l}2 \\
3 \\
4 \\
2 \\
3 \\
4 \\
5\end{array}$ & $\begin{array}{l}282148 \\
282148 \\
282139 \\
281356 \\
281357 \\
281350 \\
281405 \\
281041 \\
281054 \\
281331\end{array}$ & $\begin{array}{l}821130 \\
821130 \\
821152 \\
821204 \\
821215 \\
821230 \\
821236 \\
822415 \\
822415 \\
824352\end{array}$ & $\begin{array}{r}27 \\
27 \\
34 \\
10 \\
10 \\
9 \\
9 \\
33 \\
33 \\
17\end{array}$ & $\begin{array}{l}24 \\
24 \\
24 \\
26 \\
26 \\
26 \\
26 \\
26 \\
26 \\
26\end{array}$ & $\begin{array}{l}21 \\
21 \\
21 \\
21 \\
21 \\
21 \\
21 \\
19 \\
19 \\
16\end{array}$ & $\begin{array}{r}500,000 \\
500,000 \\
1,400,000 \\
195,000 \\
195,000 \\
260,000 \\
260,000 \\
165,000 \\
165,000 \\
225,000\end{array}$ & $\begin{array}{l}400 \\
400 \\
492 \\
400 \\
200 \\
200 \\
450\end{array}$ & $\begin{array}{r}8 \\
8 \\
8 \\
6 \\
4 \\
4 \\
10 \\
12 \\
12 \\
10\end{array}$ \\
\hline $\begin{array}{l}00517 \\
00518 \\
00519 \\
00520 \\
00521 \\
00522 \\
00561 \\
00683 \\
00684 \\
00685\end{array}$ & $\begin{array}{l}\text { ALOHA UTILITIES INC. } \\
\text { ALOHA UTILITIES INC. } \\
\text { ALOHA UTILITIES INC. } \\
\text { ALOHA UTILITIES INC. } \\
\text { ALOHA UTILITIES INC. } \\
\text { ALORA UTILITIES INC. } \\
\text { BARTELT SUNSEINE CORP } \\
\text { PASCO COUNTY } \\
\text { PASCO COUNTY } \\
\text { PASCO COUNTY }\end{array}$ & $\begin{array}{l}0318201 \\
0318201 \\
0318201 \\
0318201 \\
0318201 \\
0318201 \\
0359001 \\
0426901 \\
0426901 \\
0426901\end{array}$ & $\begin{array}{l}3 \\
4 \\
1 \\
2 \\
6 \\
7 \\
3 \\
7 \\
8 \\
9\end{array}$ & $\begin{array}{l}281222 \\
281222 \\
281145 \\
281135 \\
281332 \\
281326 \\
281122 \\
281247 \\
281247 \\
281247\end{array}$ & $\begin{array}{l}824024 \\
824015 \\
823814 \\
823705 \\
823930 \\
823921 \\
824429 \\
824311 \\
824307 \\
824306\end{array}$ & $\begin{array}{l}23 \\
23 \\
30 \\
29 \\
13 \\
13 \\
30 \\
20 \\
20 \\
20\end{array}$ & $\begin{array}{l}26 \\
26 \\
26 \\
26 \\
26 \\
26 \\
26 \\
26 \\
26 \\
26\end{array}$ & $\begin{array}{l}16 \\
16 \\
17 \\
17 \\
16 \\
16 \\
16 \\
16 \\
16 \\
16\end{array}$ & $\begin{array}{l}110,000 \\
110,000 \\
330,000 \\
330,000 \\
110,000 \\
110,000 \\
130,000 \\
230,000 \\
230,000 \\
230,000\end{array}$ & $\begin{array}{l}350 \\
350 \\
280 \\
500 \\
305 \\
302 \\
105 \\
186 \\
137 \\
180\end{array}$ & $\begin{array}{r}6 \\
6 \\
10 \\
8 \\
8 \\
8 \\
8 \\
12 \\
8 \\
8\end{array}$ \\
\hline $\begin{array}{l}00962 \\
00963 \\
01299 \\
01307 \\
01308 \\
01309 \\
01320\end{array}$ & $\begin{array}{r}\text { PASCO COUNTY } \\
\text { SAN ANTONIO, CITY OF } \\
\text { SAN ANTONIO, CITY OF } \\
\text { UTIIITIES INC. OF FLORIDA } \\
\text { UTILITIES INC. OF FLORIDA } \\
\text { PASCO COUNTY } \\
\text { ZEPHYRHILLS, CITY OF } \\
\text { ZEPHYRHILLS, CITY OF } \\
\text { ZEPHYRHILLS, CITY OF } \\
\text { PASCO COUNTY }\end{array}$ & $\begin{array}{l}0428901 \\
0455002 \\
0455002 \\
0466801 \\
0466801 \\
0601102 \\
0604002 \\
0604002 \\
0604002 \\
0612500\end{array}$ & $\begin{array}{l}1 \\
2 \\
3 \\
3 \\
4 \\
5 \\
1\end{array}$ & $\begin{array}{l}281252 \\
282013 \\
282007 \\
281141 \\
281143 \\
282534 \\
281353 \\
281432 \\
281450 \\
281023\end{array}$ & $\begin{array}{l}824346 \\
821624 \\
821632 \\
824404 \\
824412 \\
821128 \\
821007 \\
821130 \\
821112 \\
823533\end{array}$ & $\begin{array}{r}20 \\
2 \\
2 \\
30 \\
30 \\
3 \\
12 \\
10 \\
2 \\
34\end{array}$ & $\begin{array}{l}26 \\
25 \\
25 \\
26 \\
26 \\
24 \\
26 \\
26 \\
26 \\
26\end{array}$ & $\begin{array}{l}16 \\
20 \\
20 \\
16 \\
16 \\
21 \\
21 \\
21 \\
21 \\
17\end{array}$ & $\begin{array}{r}100,000 \\
231,000 \\
231,000 \\
120,000 \\
105,000 \\
125,000 \\
121,000 \\
154,000 \\
1,006,000 \\
220,000\end{array}$ & $\begin{array}{l}840 \\
460 \\
885 \\
200\end{array}$ & $\begin{array}{r}8 \\
8 \\
10 \\
12 \\
12 \\
16 \\
16 \\
16 \\
16 \\
12\end{array}$ \\
\hline $\begin{array}{l}01406 \\
01468 \\
01546 \\
01547 \\
01566\end{array}$ & $\begin{array}{r}\text { QUAIL HOLLOW UTILITY CO } \\
\text { INTERNATIONAL COMUNITY CORP } \\
\text { PASCO COUNTY } \\
\text { PASCO COUNTY } \\
\text { SCARECROW UTILITY INC. } \\
\text { SCARECROW UTILITY INC. } \\
\text { VILLAGE-TAMPA INC. THE } \\
\text { VILLAGE-TAMPA INC. THE } \\
\text { MERTZ, ESTATE OF HAROLD E. } \\
\text { MERTZ, ESTATE OF HAROLD E. }\end{array}$ & $\begin{array}{l}0646000 \\
0653900 \\
0668801 \\
0668801 \\
0681102 \\
0681102 \\
0686700 \\
0686700 \\
0742300 \\
0742300\end{array}$ & $\begin{array}{r}2 \\
2 \\
3 \\
1 \\
\text { P1 }\end{array}$ & $\begin{array}{l}281325 \\
281053 \\
281352 \\
281040 \\
281039 \\
281041 \\
281504 \\
281504 \\
281402 \\
281433\end{array}$ & $\begin{array}{l}822248 \\
822025 \\
822003 \\
821827 \\
822741 \\
822741 \\
820808 \\
820808 \\
822549 \\
822608\end{array}$ & $\begin{array}{r}14 \\
31 \\
8 \\
33 \\
36 \\
36 \\
5 \\
5 \\
8 \\
8\end{array}$ & $\begin{array}{l}26 \\
26 \\
26 \\
26 \\
26 \\
26 \\
26 \\
26 \\
26 \\
26\end{array}$ & $\begin{array}{l}19 \\
20 \\
20 \\
20 \\
18 \\
18 \\
22 \\
22 \\
19 \\
19\end{array}$ & $\begin{array}{r}250,000 \\
1,000,000 \\
150,000 \\
150,000 \\
131,000 \\
141,000 \\
120,000 \\
120,000 \\
450,000 \\
450,000\end{array}$ & $\begin{array}{l}900 \\
605 \\
425 \\
300 \\
350 \\
345 \\
345\end{array}$ & $\begin{array}{r}10 \\
16 \\
10 \\
12 \\
8 \\
10 \\
6 \\
6 \\
12 \\
12\end{array}$ \\
\hline $\begin{array}{l}01702 \\
01703 \\
01704 \\
01705 \\
01706 \\
01743 \\
01745\end{array}$ & $\begin{array}{r}\text { MERTZ, ESTATE OF HAROLD E. } \\
\text { MERTZ, ESTATE OF HAROLD } \\
\text { MERTZ. } \\
\text { MERTATE OF HAROLD } \\
\text { MERT. } \\
\text { MERTZ, ESTATE OF HAROLD E. } \\
\text { PSTA OF HAROLD E. } \\
\text { PASCO COUNTY } \\
\text { PASCO COUNTY } \\
\text { IRST PASCO SERVICE CORPORATION } \\
\text { IRST PASCO SERVICE CORPORATION } \\
\text { BETMAR UTILITIES } \\
\text { HILLVES, INC }\end{array}$ & $\begin{array}{l}0742300 \\
0742300 \\
0742300 \\
0742300 \\
0742300 \\
0759300 \\
0759500 \\
0811200 \\
0811200 \\
0837700 \\
0849100\end{array}$ & $\begin{array}{l}3 \\
4 \\
5 \\
6 \\
7 \\
1 \\
1\end{array}$ & $\begin{array}{l}281430 \\
281527 \\
281525 \\
281605 \\
281613 \\
281402 \\
281421 \\
281949 \\
281949 \\
281420 \\
281320\end{array}$ & $\begin{array}{l}822526 \\
822552 \\
822642 \\
822607 \\
822655 \\
821302 \\
821408 \\
821847 \\
821847 \\
821250 \\
821210\end{array}$ & $\begin{array}{r}8 \\
5 \\
6 \\
32 \\
31 \\
9 \\
8 \\
9 \\
9 \\
9 \\
15\end{array}$ & $\begin{array}{l}26 \\
26 \\
26 \\
25 \\
25 \\
26 \\
26 \\
25 \\
25 \\
26 \\
26\end{array}$ & $\begin{array}{l}19 \\
19 \\
19 \\
19 \\
19 \\
21 \\
21 \\
20 \\
20 \\
21 \\
21\end{array}$ & $\begin{array}{l}450,000 \\
450,000 \\
450,000 \\
450,000 \\
450,000 \\
200,000 \\
200,000 \\
138,000 \\
137,000 \\
100,000 \\
248,000\end{array}$ & 200 & $\begin{array}{r}12 \\
12 \\
12 \\
12 \\
12 \\
8 \\
8 \\
12 \\
12 \\
6 \\
10\end{array}$ \\
\hline
\end{tabular}


Table 3. - Computation of vertical travel time and radius of well protection zones for wells in the leaky confined area-Continued

\begin{tabular}{|c|c|c|c|c|c|c|c|c|c|c|c|c|c|c|}
\hline Well & $\begin{array}{l}\text { Lsd } \\
(8 e a \\
\text { lev.) }\end{array}$ & $\begin{array}{l}\text { F1 } \\
\left(\begin{array}{l}\text { sea } \\
\text { lev. }\end{array}\right.\end{array}$ & $\begin{array}{l}m \\
\text { (ft) }\end{array}$ & $\begin{array}{c}T \\
\left(f t^{2} / d\right)\end{array}$ & $\begin{array}{c}h \\
(f t)\end{array}$ & $\begin{array}{l}\text { WT } \\
(s e a \\
\text { lev. }\end{array}$ & $\begin{array}{l}\text { Pot } \\
(\text { sea } \\
\text { lev, })\end{array}$ & $\begin{array}{c}s \\
(f t)\end{array}$ & $\begin{array}{l}s(K) \\
(f t)\end{array}$ & $\begin{array}{l}\Delta h \\
(f t)\end{array}$ & $\begin{array}{c}l \\
(f t)\end{array}$ & $\begin{array}{c}\text { Leak } \\
(f t / d / f t)\end{array}$ & $\begin{array}{c}t_{v} \\
\text { (deys) }\end{array}$ & $\begin{array}{r}r \\
\text { (ft) }\end{array}$ \\
\hline & & \multicolumn{13}{|c|}{ Pasco County } \\
\hline $\begin{array}{l}00032 \\
00033 \\
00115 \\
00120 \\
00122 \\
00125 \\
00126 \\
00127 \\
00128 \\
00221\end{array}$ & $\begin{array}{r}87 \\
87 \\
97 \\
13 \\
15 \\
20 \\
25 \\
65 \\
76 \\
116\end{array}$ & $\begin{array}{r}10 \\
10 \\
60 \\
-10 \\
-10 \\
-10 \\
-10 \\
-10 \\
-10 \\
60\end{array}$ & $\begin{array}{l}960 \\
960 \\
890 \\
960 \\
970 \\
970 \\
970 \\
970 \\
970 \\
890\end{array}$ & $\begin{array}{r}42,000 \\
42,000 \\
500,000 \\
57,000 \\
57,000 \\
57,000 \\
57,000 \\
42,000 \\
42,000 \\
500,000\end{array}$ & $\begin{array}{r}588 \\
543 \\
424 \\
62 \\
75 \\
140 \\
125 \\
500 \\
474 \\
344\end{array}$ & $\begin{array}{r}72 \\
72 \\
75 \\
6 \\
5 \\
5 \\
5 \\
62 \\
71 \\
80\end{array}$ & $\begin{array}{r}61 \\
60 \\
65 \\
4 \\
5 \\
5 \\
5 \\
53 \\
57 \\
65\end{array}$ & $\begin{array}{l}0.56 \\
0.58 \\
0.96 \\
0.50 \\
0.43 \\
0.49 \\
0.41 \\
0.66 \\
0.66 \\
0.25\end{array}$ & $\begin{array}{l}1 \\
1 \\
2 \\
6 \\
4 \\
3 \\
2 \\
1 \\
1 \\
1\end{array}$ & $\begin{array}{r}12 \\
13 \\
12 \\
8 \\
4 \\
3 \\
2 \\
10 \\
15 \\
16\end{array}$ & $\begin{array}{l}62 \\
62 \\
15 \\
16 \\
15 \\
15 \\
15 \\
72 \\
81 \\
20\end{array}$ & $\begin{array}{l}0.000760 \\
0.000760 \\
0.000270 \\
0.000300 \\
0.000300 \\
0.000300 \\
0.000300 \\
0.000760 \\
0.000760 \\
0.000270\end{array}$ & $\begin{array}{r}960 \\
906 \\
654 \\
821 \\
1,111 \\
1,250 \\
1,429 \\
1,263 \\
1,066 \\
705\end{array}$ & $\begin{array}{r}540 \\
570 \\
2,710 \\
1,790 \\
1,510 \\
1,200 \\
1,170 \\
640 \\
660 \\
1,500\end{array}$ \\
\hline $\begin{array}{l}00222 \\
00223 \\
00224 \\
00256 \\
00257 \\
00258 \\
00259\end{array}$ & $\begin{array}{r}116 \\
116 \\
145 \\
87 \\
87 \\
87 \\
86 \\
68 \\
68 \\
25\end{array}$ & $\begin{array}{r}60 \\
60 \\
60 \\
50 \\
50 \\
50 \\
50 \\
20 \\
20 \\
-10\end{array}$ & $\begin{array}{l}890 \\
890 \\
890 \\
940 \\
940 \\
940 \\
940 \\
970 \\
970 \\
950\end{array}$ & $\begin{array}{r}500,000 \\
500,000 \\
500,000 \\
100,000 \\
100,000 \\
100,000 \\
100,000 \\
42,000 \\
42,000 \\
57,000\end{array}$ & $\begin{array}{l}344 \\
344 \\
407 \\
363 \\
163 \\
163 \\
414\end{array}$ & $\begin{array}{l}80 \\
80 \\
80 \\
80 \\
80 \\
80 \\
80 \\
49 \\
49 \\
3\end{array}$ & $\begin{array}{r}65 \\
65 \\
65 \\
75 \\
75 \\
75 \\
76 \\
52 \\
53 \\
3\end{array}$ & $\begin{array}{l}0.25 \\
0.25 \\
0.69 \\
0.46 \\
0.47 \\
0.63 \\
0.58 \\
0.83 \\
0.83 \\
0.86\end{array}$ & $\begin{array}{l}1 \\
1 \\
1 \\
1 \\
2 \\
3 \\
1\end{array}$ & $\begin{array}{r}16 \\
16 \\
16 \\
6 \\
7 \\
8 \\
5\end{array}$ & $\begin{array}{l}20 \\
20 \\
20 \\
30 \\
30 \\
30 \\
30 \\
29 \\
29 \\
13\end{array}$ & $\begin{array}{l}0.000270 \\
0.000270 \\
0.000270 \\
0.000130 \\
0.000130 \\
0.000130 \\
0.000130 \\
0.000760 \\
0.000360 \\
0.000360\end{array}$ & $\begin{array}{r}705 \\
705 \\
705 \\
4,196 \\
3,846 \\
3,550 \\
4,615 \\
\\
481\end{array}$ & $\stackrel{\star}{\star}, 520$ \\
\hline $\begin{array}{l}00517 \\
00518 \\
00519 \\
00520 \\
00521 \\
00522 \\
00561 \\
00683 \\
00684 \\
00685\end{array}$ & $\begin{array}{l}26 \\
26 \\
39 \\
43 \\
26 \\
26 \\
17 \\
35 \\
46 \\
48\end{array}$ & $\begin{array}{r}-10 \\
-10 \\
0 \\
0 \\
-10 \\
-10 \\
-10 \\
-10 \\
-10 \\
-10\end{array}$ & $\begin{array}{l}960 \\
960 \\
970 \\
970 \\
950 \\
950 \\
970 \\
950 \\
950 \\
950\end{array}$ & $\begin{array}{l}57,000 \\
57,000 \\
57,000 \\
57,000 \\
57,000 \\
57,000 \\
57,000 \\
57,000 \\
57,000 \\
57,000\end{array}$ & $\begin{array}{r}314 \\
314 \\
241 \\
457 \\
269 \\
266 \\
78 \\
141 \\
81 \\
122\end{array}$ & $\begin{array}{r}12 \\
12 \\
28 \\
37 \\
21 \\
21 \\
5 \\
6 \\
6 \\
6\end{array}$ & $\begin{array}{r}16 \\
16 \\
24 \\
29 \\
21 \\
21 \\
4 \\
4 \\
4 \\
4\end{array}$ & $\begin{array}{l}0.44 \\
0.44 \\
1.26 \\
1.29 \\
0.43 \\
0.43 \\
0.51 \\
0.86 \\
0.90 \\
0.90\end{array}$ & $\begin{array}{l}1 \\
1 \\
4 \\
2 \\
1 \\
1 \\
5 \\
5 \\
8 \\
6\end{array}$ & $\begin{array}{r}-3 \\
-3 \\
8 \\
10 \\
1 \\
1 \\
6 \\
7 \\
10 \\
8\end{array}$ & $\begin{array}{l}22 \\
22 \\
28 \\
37 \\
31 \\
31 \\
15 \\
16 \\
16 \\
16\end{array}$ & $\begin{array}{l}0.000300 \\
0.000300 \\
0.000300 \\
0.000300 \\
0.000300 \\
0.000300 \\
0.000300 \\
0.000300 \\
0.000300 \\
0.000300\end{array}$ & $\begin{array}{r}7,333 \\
7,333 \\
1,436 \\
1,644 \\
3,444 \\
3,444 \\
909 \\
889 \\
711 \\
821\end{array}$ & $\begin{array}{l}1,460 \\
1,060 \\
\\
1,610 \\
1,590 \\
2,100 \\
1,710\end{array}$ \\
\hline 00688 & $\begin{array}{r}35 \\
150 \\
158\end{array}$ & $\begin{array}{r}-10 \\
100 \\
90\end{array}$ & $\begin{array}{r}950 \\
890 \\
1,201\end{array}$ & $\begin{array}{l}57,000 \\
40,000 \\
40,000\end{array}$ & 92 & $\begin{array}{r}4 \\
120 \\
115\end{array}$ & $\begin{array}{r}3 \\
80 \\
80\end{array}$ & $\begin{array}{l}0.39 \\
1.26 \\
1.23\end{array}$ & 3 & 4 & $\begin{array}{l}14 \\
20 \\
25\end{array}$ & $\begin{array}{l}0.000360 \\
0.000130 \\
0.000130\end{array}$ & 864 & $\begin{array}{c}1,300 \\
\star \star \\
\star \star\end{array}$ \\
\hline $\begin{array}{l}00962 \\
00963 \\
01299 \\
01307 \\
01308 \\
01309 \\
01320\end{array}$ & $\begin{array}{r}16 \\
15 \\
86 \\
105 \\
94 \\
98 \\
49\end{array}$ & $\begin{array}{r}-10 \\
-10 \\
50 \\
50 \\
50 \\
50 \\
5\end{array}$ & $\begin{array}{l}960 \\
960 \\
880 \\
950 \\
940 \\
940 \\
980\end{array}$ & $\begin{array}{r}57,000 \\
57,000 \\
500,000 \\
100,000 \\
100,000 \\
100,000 \\
57,000\end{array}$ & $\begin{array}{r}134 \\
92 \\
\\
785 \\
416 \\
837 \\
156\end{array}$ & $\begin{array}{r}5 \\
5 \\
70 \\
80 \\
87 \\
90 \\
45\end{array}$ & $\begin{array}{r}3 \\
3 \\
62 \\
66 \\
66 \\
65 \\
33\end{array}$ & $\begin{array}{l}0.45 \\
0.39 \\
0.06 \\
0.26 \\
0.33 \\
2.15 \\
0.82\end{array}$ & $\begin{array}{l}2 \\
3 \\
0 \\
1 \\
2 \\
4\end{array}$ & $\begin{array}{r}4 \\
5 \\
14 \\
22 \\
27 \\
16\end{array}$ & $\begin{array}{l}15 \\
15 \\
20 \\
30 \\
37 \\
40 \\
40\end{array}$ & $\begin{array}{l}0.000300 \\
0.000300 \\
0.000130 \\
0.000130 \\
0.000130 \\
0.000130 \\
0.000300\end{array}$ & $\begin{array}{r}1,111 \\
1,000 \\
>1,825 \\
2,429 \\
2,108 \\
1,923 \\
1,270\end{array}$ & $\begin{array}{l}1,180 \\
1,330\end{array}$ \\
\hline $\begin{array}{l}01406 \\
01468 \\
01546 \\
01547 \\
01566\end{array}$ & $\begin{array}{l}65 \\
55 \\
77 \\
73 \\
69 \\
69\end{array}$ & $\begin{array}{l}30 \\
30 \\
50 \\
40 \\
10 \\
10\end{array}$ & $\begin{array}{l}950 \\
970 \\
940 \\
970 \\
980 \\
980\end{array}$ & $\begin{array}{l}42,000 \\
42,000 \\
42,000 \\
42,000 \\
26,000 \\
26,000\end{array}$ & $\begin{array}{l}865 \\
580 \\
398 \\
267 \\
291\end{array}$ & $\begin{array}{l}56 \\
53 \\
75 \\
61 \\
68 \\
68\end{array}$ & $\begin{array}{l}60 \\
56 \\
71 \\
61 \\
60 \\
60\end{array}$ & $\begin{array}{l}1.27 \\
4.86 \\
0.76 \\
0.75 \\
1.08 \\
1.13\end{array}$ & $\begin{array}{l}1 \\
7 \\
2 \\
2 \\
3\end{array}$ & $\begin{array}{r}-3 \\
4 \\
6 \\
2 \\
11\end{array}$ & $\begin{array}{l}26 \\
23 \\
25 \\
21 \\
58 \\
58\end{array}$ & $\begin{array}{l}0.000300 \\
0.000300 \\
0.000150 \\
0.000150 \\
0.000760 \\
0.000760\end{array}$ & $\begin{array}{r}8,667 \\
1,704 \\
3,030 \\
4,000 \\
954\end{array}$ & $\begin{array}{l}840 \\
\star\end{array}$ \\
\hline $\begin{array}{l}01574 \\
01575 \\
01700 \\
01701\end{array}$ & $\begin{array}{l}82 \\
82 \\
71 \\
76\end{array}$ & $\begin{array}{l}60 \\
60 \\
30 \\
30\end{array}$ & $\begin{array}{l}940 \\
940 \\
940 \\
940\end{array}$ & $\begin{array}{r}100,000 \\
100,000 \\
26,000 \\
26,000\end{array}$ & $\begin{array}{l}323 \\
323\end{array}$ & $\begin{array}{l}80 \\
80 \\
66 \\
68\end{array}$ & $\begin{array}{l}70 \\
70 \\
59 \\
62\end{array}$ & $\begin{array}{l}0.28 \\
0.28 \\
3.55 \\
3.55\end{array}$ & $\begin{array}{l}1 \\
1\end{array}$ & $\begin{array}{l}11 \\
11\end{array}$ & $\begin{array}{l}20 \\
20 \\
36 \\
38\end{array}$ & $\begin{array}{l}0.000270 \\
0.000270 \\
0.000760 \\
0.000760\end{array}$ & $\begin{array}{l}926 \\
926\end{array}$ & $\begin{array}{l}760 \\
760 \\
\star \star \\
\star \star\end{array}$ \\
\hline $\begin{array}{l}01702 \\
01703 \\
01704 \\
01705 \\
01706 \\
01743 \\
01745\end{array}$ & $\begin{array}{r}58 \\
67 \\
74 \\
75 \\
76 \\
87 \\
103 \\
95 \\
95 \\
93 \\
88\end{array}$ & $\begin{array}{l}30 \\
30 \\
30 \\
30 \\
20 \\
50 \\
50 \\
60 \\
60 \\
50 \\
50\end{array}$ & $\begin{array}{l}940 \\
930 \\
930 \\
920 \\
920 \\
940 \\
940 \\
890 \\
890 \\
940 \\
950\end{array}$ & $\begin{array}{r}42,000 \\
42,000 \\
26,000 \\
26,000 \\
26,000 \\
100,000 \\
50,000 \\
86,000 \\
86,000 \\
100,000 \\
100,000\end{array}$ & 147 & $\begin{array}{l}58 \\
58 \\
72 \\
70 \\
73 \\
80 \\
85 \\
85 \\
85 \\
80 \\
78\end{array}$ & $\begin{array}{l}58 \\
60 \\
70 \\
66 \\
70 \\
77 \\
81 \\
78 \\
78 \\
77 \\
74\end{array}$ & $\begin{array}{l}2.25 \\
2.25 \\
3.55 \\
3.55 \\
3.55 \\
0.46 \\
0.88 \\
0.35 \\
0.35 \\
0.23 \\
0.55\end{array}$ & 5 & 9 & $\begin{array}{l}28 \\
28 \\
42 \\
40 \\
53 \\
30 \\
35 \\
25 \\
25 \\
30 \\
28\end{array}$ & $\begin{array}{l}0.000760 \\
0.000760 \\
0.000760 \\
0.000760 \\
0.000760 \\
0.000130 \\
0.000130 \\
0.000061 \\
0.000061 \\
0.000130 \\
0.000130\end{array}$ & $\begin{array}{r}>1,825 \\
3,846 \\
>1,825 \\
>1,825 \\
>1,825 \\
>1,825\end{array}$ & $\begin{array}{l}\star \\
\star \\
\star \\
\star\end{array}$ \\
\hline
\end{tabular}


Table 3. - Computation of vertical travel time and radius of well protection zones for wells in the leaky confined area-Continued

\begin{tabular}{|c|c|c|c|c|c|c|c|c|c|}
\hline Well & Omer & $\begin{array}{c}\text { Cons. } \\
\text { use } \\
\text { permit }\end{array}$ & $\begin{array}{l}\text { Well } \\
\text { name }\end{array}$ & Lat. & Long. Sec. & Twn; Rns; & $\begin{array}{c}\text { Av.Q } \\
(\mathrm{gal} / \mathrm{d})\end{array}$ & $\begin{array}{r}\text { Well } \\
\text { depth } \\
\text { (ft) }\end{array}$ & $\begin{array}{l}\text { Dia } \\
\text { (in) }\end{array}$ \\
\hline
\end{tabular}

Pinellas County

00171
00172
00173
00481
00482
00484
00485
00486

TARPON SPRINGS, CITY OF TARPON SPRINGS, CITY OF TARPON SPRINGS, CITY OF CLEARWATER, CITY OF CLEARWATER, CITY OF CLEARWATER, CITY OF CLEARWATER, CITY OF CLEARWATER, CITY OF

0074202
0074202
0074202
0298101
0298101
0298101
0298101
0298101

1
2
3
45
46
51
52

$\begin{array}{rrr}280843 & 824513 & 12 \\ 280846 & 824407 & 7 \\ 280822 & 824402 & 18 \\ 275752 & 824345 & 17 \\ 275751 & 824332 & 17 \\ 275751 & 824400 & 18 \\ 275747 & 824400 & 18 \\ 275741 & 824401 & 18\end{array}$

Hillsborough County
00231

00232

00233

00234

00236

00241

00242

00247

00249

00250

00251

00378

00379

00405

00406

00512

00774 WEST COAST REGIONAL WAT SUP AU

00775 WEST COAST REGIONAL WAT SUP AU

00814 WEST COAST REGIONAL WAT SUP AU

00815 WEST COAST REGIONAL WAT SUP AU

00817 WEST COAST REGIONAL WAT SUP AU

00820 WEST COAST REGIONAL WAT SUP AU

00824 WEST COAST REGIONAL WAT SUP AU

00825 WEST COAST REGIONAL WAT SUP AU

00826 WEST COAST REGIONAL WAT SUP AU

00827 WEST COAST REGIONAL WAT SUP AU

00828 WEST COAST REGIONAL WAT SUP AU

00829 WEST COAST REGIONAL WAT SUP AU 00830 WEST COAST REGIONAL WAT SUP AU 00835 WEST COAST REGIONAL WAT SUP AU CYPRESS BEND DEVELOPMENT CORP CYPRESS BEND DEVELOPMENT CORP FLORIDA CITIES WATER COMPANY FLORIDA CITIES WATER COMPANY FLORIDA CITIES WATER COMPANY $C W D$ INC.

01355 01499 WEST COAST REGIONAL WAT SUP AU 0045003 0045003 0045003 0045003 0045003 0045003 0045003

0045003 0045003 0045003

0045003 0177601 0177601 0177601 0177601

0177601

0178701

0178701

0196002

0196002

0196002

0196002

0270701

0270701

0284002

0284002

0313203

0313203

0313203

0435201

0435201

0435201

0435201

0435201

0435201

0435201

0435201

0435201

0435201

0435201

0435201

0435201

0435201

0530000

0530000

0588601

0588601

0588601

0631200

0667600

$\begin{array}{rlll}1 & 280404 & 822310 & 10 \\ 1 & 280211 & 822308 & 22 \\ 2 & 280212 & 822307 & 23 \\ 3 & 280241 & 822319 & 15 \\ 4 & 280241 & 822318 & 15 \\ 5 & 280203 & 822303 & 23 \\ 6 & 280251 & 822330 & 15 \\ 7 & 280251 & 822330 & 15 \\ 8 & 280251 & 822330 & 15 \\ 11 & 280249 & 822324 & 15\end{array}$

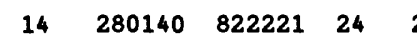

$14 \quad 280140 \quad 822221 \quad 24$

280109

280108

280042

280751

280743

280358

280359

$\begin{array}{ll}6 & 280355 \\ 7 & 280357\end{array}$

280357

275944

$11 \quad 275610$

12
4 275613

280906

280916

RUS9

274252

RS10

274252

POI

POL1

PRMI

AB5

AB6

$A B 7$

275953

280009

280017

275908

274604

274616

274624

274553

AB12

AB17

274554

274601

280015

280823

280816

280307

280310

280233

280633

CC- 1 280441 $\begin{array}{ll}820719 & 29 \\ 820720 & 29\end{array}$

82072529

$820551 \quad 22$

$\begin{array}{ll}822625 & 18 \\ 822633 & 18\end{array}$

1827

822451

822452

822452

822251

8222522

$822146 \quad 25$

82213325

822105

8220157

82231710

82232410

$821833 \quad 33$

$821651 \quad 35$

$821636 \quad 35$

$821826 \quad 4$

82233222

82232922

82233722

82233822

82233322

82234122

82161735

$823309 \quad 13$

$823319 \quad 13$

82293515

8229581

82381530

822935

$\begin{array}{ll}27 & 15 \\ 27 & 16 \\ 27 & 16 \\ 29 & 16 \\ 29 & 16 \\ 29 & 16 \\ 29 & 16 \\ 29 & 16\end{array}$

15
16
16
16
16
16
16

100,000

550,000

100,000

472,500

306,500

423,200

380,666

269,000

$\begin{array}{ll}28 & 19 \\ 28 & 19 \\ 28 & 19 \\ 28 & 19 \\ 28 & 19 \\ 28 & 19 \\ 28 & 19 \\ 28 & 19 \\ 28 & 19 \\ 28 & 19 \\ 28 & 19 \\ 28 & 22 \\ 28 & 22 \\ 28 & 22 \\ 28 & 22 \\ 28 & 22 \\ 27 & 19 \\ 27 & 19 \\ 28 & 19 \\ 28 & 19\end{array}$

100,000

400,000

200,000

260,000

640,000

650,000

400,000

400,000

300,000

640,000

160,000

800,000

800,000

800,000

900,000

$2,900,000$

400,000

400,000

170,000

230,000

150,000

160,000

427,000

427,000

160,000

160,000

151,000

308,700

308,700

350,000

350,000

500,000

250,000

400,000

450,000

108,000

108,000

108,000

123,000

123,000

123,000

123,000

770,000

182,500

182,500

400,000

400,000

420,000

116,000

157,000

$\begin{array}{ll}110 & 10 \\ 110 & 10 \\ 110 & 10 \\ 290 & 10 \\ 290 & 10 \\ 200 & 12 \\ 200 & 12 \\ 240 & 12\end{array}$

200

10
10
10
10
10
12
12
12

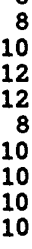

676

367
760

76010

$650 \quad 12$
$1,200 \quad 18$

5858

5858

$\begin{array}{ll}350 & 12 \\ 300 & 12\end{array}$

$350 \quad 12$

$340 \quad 12$

$537 \quad 10$

560

12

80010

$700 \quad 10$

2158

$535 \quad 12$

$470 \quad 10$

$600 \quad 8$

$\begin{array}{ll}400 & 6 \\ 400 & 6\end{array}$

$280 \quad 8$

315

$340 \quad 8$

$300 \quad 6$

$600 \quad 16$

12

8
12

$401 \quad 12$

352
8 
Table 3. - Computation of vertical travel time and radius of well protection zones for wells in the leaky confined area-Continued

\begin{tabular}{|c|c|c|c|c|c|c|c|c|c|c|c|c|c|c|}
\hline Well & $\begin{array}{l}\text { Lsd } \\
\text { (sea } \\
\text { lev, })\end{array}$ & $\begin{array}{l}\text { F1 } \\
(\mathrm{sea} \\
\text { lev.) }\end{array}$ & $\stackrel{m}{f t}$ & $\begin{array}{c}T \\
\left(\mathrm{ft}^{2} / \mathrm{d}\right)\end{array}$ & $\begin{array}{c}h \\
(f t)\end{array}$ & $\begin{array}{l}\text { WI } \\
(s e a \\
\text { lev.) }\end{array}$ & $\begin{array}{l}\text { Pot } \\
(\text { sea } \\
\text { lev.) }\end{array}$ & $\begin{array}{c}s \\
\text { (ft) }\end{array}$ & $s(K)$ & $\begin{array}{l}\Delta h \\
(f t)\end{array}$ & $\begin{array}{c}l \\
(f t)\end{array}$ & $\begin{array}{c}\text { Leak } \\
(\mathrm{ft} / \mathrm{d} / \mathrm{ft})\end{array}$ & $\begin{array}{c}t_{v} \\
\text { (days) }\end{array}$ & $\underset{(f t)}{r}$ \\
\hline & & & & & & & Pinel & las Co & unty & & & & & \\
\hline \multirow[t]{2}{*}{$\begin{array}{l}00171 \\
00172 \\
00173 \\
00481 \\
00482 \\
00484 \\
00485 \\
00486\end{array}$} & $\begin{array}{l}20 \\
25 \\
24 \\
48 \\
47 \\
55 \\
58 \\
60\end{array}$ & $\begin{array}{r}0 \\
0 \\
0 \\
-20 \\
-20 \\
-20 \\
-20 \\
-20\end{array}$ & $\begin{array}{r}990 \\
990 \\
1,000 \\
1,120 \\
1,120 \\
1,120 \\
1,120 \\
1,120\end{array}$ & $\begin{array}{l}57,000 \\
57,000 \\
57,000 \\
70,000 \\
70,000 \\
70,000 \\
70,000 \\
70,000\end{array}$ & $\begin{array}{r}90 \\
85 \\
86 \\
222 \\
223 \\
125 \\
122 \\
160\end{array}$ & $\begin{array}{r}5 \\
5 \\
4 \\
35 \\
32 \\
37 \\
37 \\
37\end{array}$ & $\begin{array}{l}3 \\
5 \\
5 \\
5 \\
4 \\
5 \\
5 \\
5\end{array}$ & $\begin{array}{l}0.38 \\
2.10 \\
0.38 \\
1.48 \\
0.96 \\
1.30 \\
1.17 \\
0.83\end{array}$ & $\begin{array}{r}3 \\
18 \\
3 \\
6 \\
4 \\
9 \\
8 \\
5\end{array}$ & $\begin{array}{r}5 \\
18 \\
2 \\
36 \\
32 \\
41 \\
40 \\
37\end{array}$ & $\begin{array}{r}5 \\
5 \\
4 \\
55 \\
52 \\
57 \\
57 \\
57\end{array}$ & $\begin{array}{l}0.000300 \\
0.000300 \\
0.000300 \\
0.000013 \\
0.000013 \\
0.000013 \\
0.000013 \\
0.000013\end{array}$ & $\begin{array}{r}333 \\
145 \\
381 \\
20,638 \\
21,622 \\
19,064 \\
19,487 \\
20,879\end{array}$ & $\begin{array}{l}1,310 \\
3,170 \\
1,340\end{array}$ \\
\hline & & \multicolumn{13}{|c|}{ Hillsborough County } \\
\hline 00112 & $\begin{array}{l}37 \\
65 \\
67 \\
80 \\
75 \\
60 \\
65 \\
65 \\
65 \\
78\end{array}$ & $\begin{array}{r}-10 \\
0 \\
0 \\
0 \\
0 \\
0 \\
0 \\
0 \\
0 \\
0\end{array}$ & $\begin{array}{l}1,080 \\
1,110 \\
1,110 \\
1,110 \\
1,100 \\
1,120 \\
1,100 \\
1,100 \\
1,100 \\
1,100\end{array}$ & $\begin{array}{l}240,000 \\
240,000 \\
240,000 \\
240,000 \\
240,000 \\
240,000 \\
240,000 \\
240,000 \\
240,000 \\
240,000\end{array}$ & 153 & $\begin{array}{l}25 \\
29 \\
29 \\
40 \\
40 \\
28 \\
48 \\
48 \\
48 \\
43\end{array}$ & $\begin{array}{l}25 \\
20 \\
20 \\
20 \\
20 \\
20 \\
21 \\
21 \\
21 \\
20\end{array}$ & $\begin{array}{l}0.10 \\
0.40 \\
0.19 \\
0.25 \\
0.61 \\
0.64 \\
0.39 \\
0.39 \\
0.29 \\
0.62\end{array}$ & 1 & 1 & $\begin{array}{l}35 \\
29 \\
29 \\
40 \\
40 \\
28 \\
48 \\
48 \\
48 \\
43\end{array}$ & $\begin{array}{l}0.000610 \\
0.000300 \\
0.000300 \\
0.000300 \\
0.000300 \\
0.000300 \\
0.000300 \\
0.000300 \\
0.000300 \\
0.000300\end{array}$ & 1,913 & $\begin{array}{l}\star \\
\star \\
\star \star \\
\star \star \\
\star \\
\star \star \\
\star \star \\
\star \star \\
\star \star \\
\star \star\end{array}$ \\
\hline $\begin{array}{l}00231 \\
00232 \\
00233 \\
00234 \\
00236 \\
00241 \\
00242 \\
00247 \\
00249\end{array}$ & $\begin{array}{r}33 \\
130 \\
128 \\
130 \\
124 \\
146 \\
64 \\
64 \\
52 \\
54\end{array}$ & $\begin{array}{r}0 \\
20 \\
20 \\
20 \\
20 \\
30 \\
0 \\
0 \\
0 \\
0\end{array}$ & $\begin{array}{l}1,120 \\
1,100 \\
1,100 \\
1,100 \\
1,100 \\
1,090 \\
1,000 \\
1,010 \\
1,080 \\
1,080\end{array}$ & $\begin{array}{r}240,000 \\
130,000 \\
130,000 \\
130,000 \\
130,000 \\
130,000 \\
26,000 \\
26,000 \\
100,000 \\
100,000\end{array}$ & $\begin{array}{r}566 \\
259 \\
650 \\
546 \\
1,084 \\
521 \\
521 \\
298 \\
246\end{array}$ & $\begin{array}{r}22 \\
120 \\
120 \\
120 \\
120 \\
120 \\
53 \\
53 \\
26 \\
26\end{array}$ & $\begin{array}{l}21 \\
72 \\
72 \\
72 \\
70 \\
78 \\
52 \\
52 \\
24 \\
24\end{array}$ & $\begin{array}{l}0.16 \\
1.37 \\
1.37 \\
1.39 \\
1.54 \\
4.76 \\
3.29 \\
3.29 \\
0.37 \\
0.50\end{array}$ & $\begin{array}{l}2 \\
5 \\
2 \\
3 \\
5 \\
6 \\
6 \\
1 \\
2\end{array}$ & $\begin{array}{r}50 \\
53 \\
50 \\
53 \\
47 \\
7 \\
7 \\
3 \\
4\end{array}$ & $\begin{array}{r}22 \\
100 \\
100 \\
100 \\
100 \\
90 \\
53 \\
53 \\
26 \\
26\end{array}$ & $\begin{array}{l}0.000300 \\
0.000067 \\
0.000067 \\
0.000067 \\
0.000067 \\
0.000067 \\
0.000760 \\
0.000760 \\
0.000300 \\
0.000300\end{array}$ & $\begin{array}{l}5,427 \\
5,147 \\
5,427 \\
5,147 \\
5,166 \\
1,162 \\
1,162 \\
2,167 \\
1,926\end{array}$ & $\begin{array}{l}1,090 \\
1,090\end{array}$ \\
\hline $\begin{array}{l}00250 \\
00251 \\
00378 \\
00379 \\
00405 \\
00406 \\
00512\end{array}$ & $\begin{array}{l}48 \\
50 \\
34 \\
34 \\
26 \\
27 \\
48 \\
56 \\
55 \\
42\end{array}$ & $\begin{array}{r}0 \\
0 \\
0 \\
0 \\
-10 \\
-10 \\
20 \\
20 \\
20 \\
-120\end{array}$ & $\begin{array}{r}1,090 \\
1,080 \\
1,160 \\
1,160 \\
1,200 \\
1,200 \\
980 \\
980 \\
980 \\
1,170\end{array}$ & $\begin{array}{r}100,000 \\
100,000 \\
200,000 \\
200,000 \\
200,000 \\
200,000 \\
42,000 \\
42,000 \\
42,000 \\
130,000\end{array}$ & $\begin{array}{l}302 \\
290 \\
304 \\
503 \\
\\
278 \\
532\end{array}$ & $\begin{array}{l}26 \\
26 \\
22 \\
22 \\
20 \\
20 \\
44 \\
48 \\
50 \\
37\end{array}$ & $\begin{array}{r}24 \\
24 \\
14 \\
14 \\
9 \\
10 \\
42 \\
42 \\
44 \\
-4\end{array}$ & $\begin{array}{l}0.33 \\
0.35 \\
0.52 \\
0.49 \\
0.19 \\
0.19 \\
0.76 \\
1.54 \\
1.54 \\
0.61\end{array}$ & $\begin{array}{l}1 \\
1 \\
2 \\
1 \\
1 \\
1\end{array}$ & $\begin{array}{r}3 \\
3 \\
10 \\
9 \\
11 \\
3\end{array}$ & $\begin{array}{r}26 \\
26 \\
22 \\
22 \\
30 \\
30 \\
24 \\
28 \\
30 \\
157\end{array}$ & $\begin{array}{l}0.000300 \\
0.000300 \\
0.000067 \\
0.000067 \\
0.000007 \\
0.000007 \\
0.000300 \\
0.000300 \\
0.000300 \\
0.000013\end{array}$ & $\begin{array}{r}2,167 \\
2,167 \\
4,378 \\
4,691 \\
>1,825 \\
53,571 \\
2,000\end{array}$ & * \\
\hline $\begin{array}{l}00775 \\
00814 \\
00815 \\
00817 \\
00820 \\
00824 \\
00825 \\
00826 \\
00827 \\
00828\end{array}$ & $\begin{array}{l}42 \\
31 \\
85 \\
82 \\
77 \\
12 \\
11 \\
12 \\
12 \\
10\end{array}$ & $\begin{array}{r}-120 \\
0 \\
10 \\
10 \\
0 \\
-80 \\
-75 \\
-75 \\
-80 \\
-80\end{array}$ & $\begin{array}{l}1,170 \\
1130 \\
1,120 \\
1,100 \\
1,140 \\
1,170 \\
1,180 \\
1,180 \\
1,170 \\
1,180\end{array}$ & $\begin{array}{l}130,000 \\
200,000 \\
200,000 \\
200,000 \\
200,000 \\
130,000 \\
130,000 \\
130,000 \\
130,000 \\
130,000\end{array}$ & $\begin{array}{l}538 \\
184 \\
\\
463 \\
393 \\
508 \\
314 \\
313 \\
188 \\
225\end{array}$ & $\begin{array}{r}36 \\
25 \\
56 \\
51 \\
33 \\
8 \\
8 \\
7 \\
8 \\
7\end{array}$ & $\begin{array}{r}-4 \\
17 \\
19 \\
19 \\
17 \\
1 \\
1 \\
1 \\
1 \\
1\end{array}$ & $\begin{array}{l}0.61 \\
0.59 \\
0.29 \\
0.45 \\
0.52 \\
0.19 \\
0.20 \\
0.20 \\
0.22 \\
0.22\end{array}$ & $\begin{array}{l}1 \\
1 \\
0 \\
1 \\
1 \\
1 \\
1\end{array}$ & $\begin{array}{r}41 \\
11 \\
33 \\
17 \\
7 \\
8 \\
7 \\
8 \\
7\end{array}$ & $\begin{array}{r}156 \\
25 \\
46 \\
41 \\
33 \\
88 \\
83 \\
82 \\
88 \\
87\end{array}$ & $\begin{array}{l}0.000013 \\
0.000067 \\
0.000067 \\
0.000067 \\
0.000067 \\
0.000010 \\
0.000010 \\
0.000010 \\
0.000010 \\
0.000010\end{array}$ & $\begin{array}{r}52,174 \\
4,664 \\
>1,825 \\
3,221 \\
4,478 \\
146,667 \\
127,692 \\
136,667 \\
135,385 \\
145,000\end{array}$ & \\
\hline $\begin{array}{l}00829 \\
00830 \\
00835\end{array}$ & $\begin{array}{l}11 \\
11 \\
47 \\
56 \\
56 \\
47 \\
43 \\
43\end{array}$ & $\begin{array}{r}-80 \\
-80 \\
10 \\
0 \\
0 \\
0 \\
0 \\
0\end{array}$ & $\begin{array}{l}1,170 \\
1,170 \\
1,170 \\
1,000 \\
1,010 \\
1,110 \\
1,110 \\
1,130\end{array}$ & $\begin{array}{r}130,000 \\
130,000 \\
200,000 \\
57,000 \\
57,000 \\
57,000 \\
26,000 \\
57,000\end{array}$ & $\begin{array}{l}249 \\
209 \\
563\end{array}$ & $\begin{array}{r}8 \\
7 \\
42 \\
50 \\
49 \\
36 \\
36 \\
34\end{array}$ & $\begin{array}{r}1 \\
1 \\
20 \\
34 \\
33 \\
25 \\
25 \\
20\end{array}$ & $\begin{array}{l}0.22 \\
0.22 \\
0.85 \\
0.68 \\
0.68 \\
1.56 \\
3.15 \\
1.57\end{array}$ & $\begin{array}{l}1 \\
1 \\
2\end{array}$ & $\begin{array}{r}8 \\
7 \\
24\end{array}$ & $\begin{array}{l}88 \\
87 \\
32 \\
50 \\
49 \\
36 \\
36 \\
34\end{array}$ & $\begin{array}{l}0.000010 \\
0.000010 \\
0.000067 \\
0.000300 \\
0.000300 \\
0.000300 \\
0.000300 \\
0.000300\end{array}$ & $\begin{array}{r}135,385 \\
145,000 \\
3,294\end{array}$ & $\begin{array}{l}\star \star \\
\star \\
\star \star \\
\star \star \\
\star \star\end{array}$ \\
\hline $\begin{array}{l}01355 \\
01499\end{array}$ & $\begin{array}{l}32 \\
53\end{array}$ & $\begin{array}{r}-10 \\
0\end{array}$ & $\begin{array}{l}1,030 \\
1,080\end{array}$ & $\begin{array}{l}57,000 \\
26,000\end{array}$ & $\begin{array}{l}359 \\
299\end{array}$ & $\begin{array}{l}25 \\
45\end{array}$ & $\begin{array}{l}18 \\
35\end{array}$ & $\begin{array}{l}0.43 \\
1.33\end{array}$ & $\begin{array}{l}1 \\
4\end{array}$ & $\begin{array}{r}8 \\
14\end{array}$ & $\begin{array}{l}35 \\
45\end{array}$ & $\begin{array}{l}0.000540 \\
0.000360\end{array}$ & $\begin{array}{r}997 \\
1,316\end{array}$ & $\begin{array}{l}710 \\
900\end{array}$ \\
\hline
\end{tabular}


Table 3. - Computation of vertical travel time and radius of well protection zones for wells in the leaky confined area-Continued

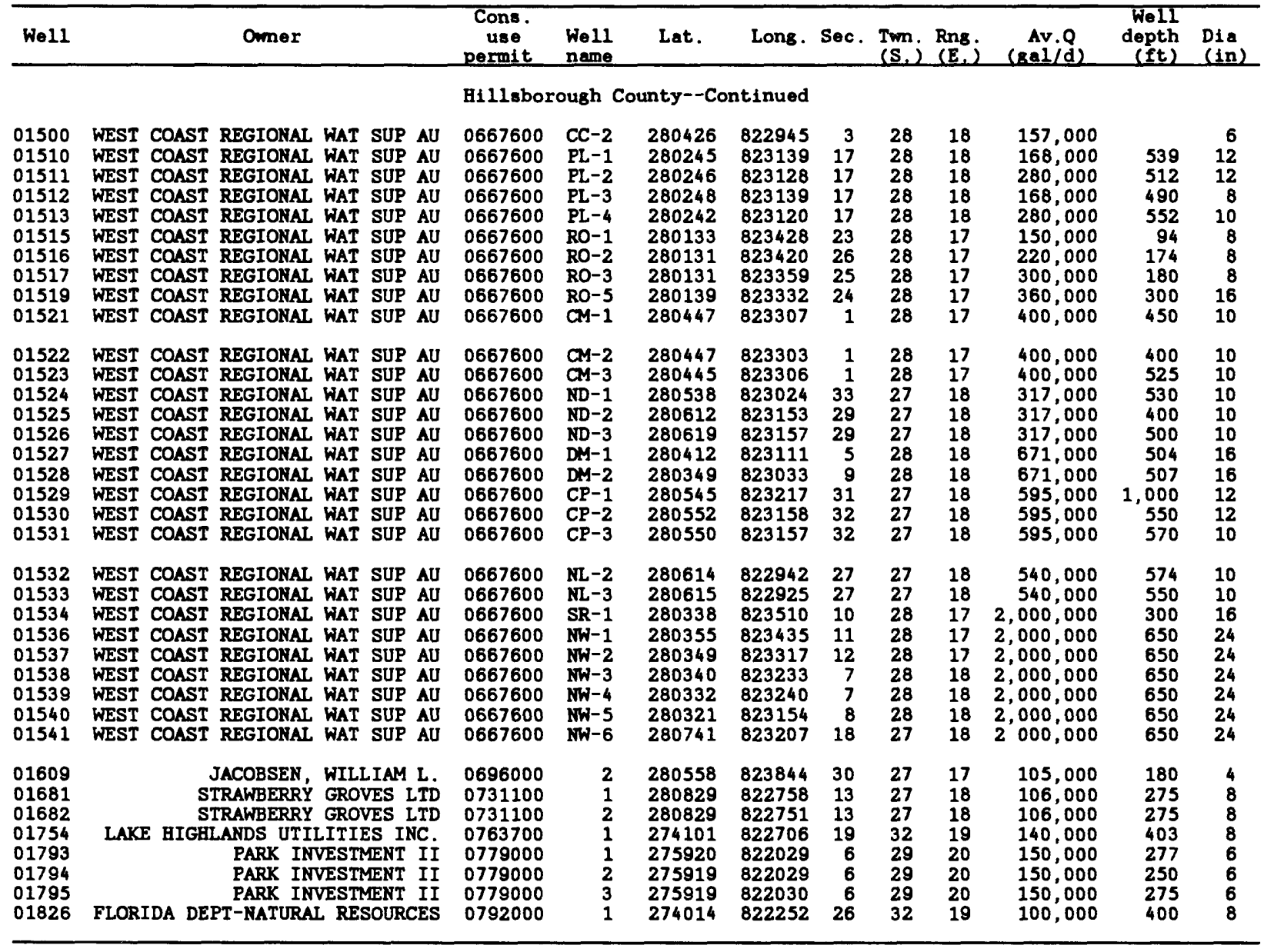

Except in large well fields, the scale of the available potentiometric-surface maps was insufficient to give accurate pumping water levels in the vicinity of municipal supply wells. For wells where pumping water levels were not available, a 1-day drawdown was calculated using the average permitted pumping rate, transmissivity at the well site, and a storage coefficient of 0.001 . Drawdowns for fully penetrating wells were calculated for one day of pumping using the modified nonequilibrium formula developed by Cooper and Jacob in 1946 (cited in Lohman, 1972, p. 19). For partially penetrating wells, a correction was made using the Kozeny formula (1933) as given by Jacob (1945), modified to show drawdown in the partially penetrating well:

$$
s(K)=\frac{s}{p\left(1+7 \sqrt{\frac{r_{w}}{2 p m}} \cos \frac{p \pi}{2}\right)},
$$

where

$$
\begin{gathered}
\mathbf{s}=\begin{array}{c}
\text { drawdown in a hypothetical fully } \\
\text { penetrating well, in feet; }
\end{array} \\
\mathbf{s}(\mathbf{K})=\text { drawdown in the partially penetrating } \\
\text { well, in feet; } \\
\mathbf{p}=\text { fraction of the aquifer penetrated, } \\
\text { expressed as a decimal; } \\
\mathbf{r}_{\mathbf{w}}=\text { radius of the well, in feet; } \\
\mathbf{m}=\text { aquifer thickness, in feet; and }
\end{gathered}
$$

the argument for cosine is in radians.

The corrected drawdown was subtracted from the altitude of the Floridan potentiometric surface, which was determined from potentiometric-surface maps, and the resulting value was subtracted from the altitude of the water table to obtain the estimated head difference $(\Delta \mathrm{h})$ under pumping conditions, as shown in table 3. The value of $\Delta h$ was increased by 5 feet, or half of a contour interval on the potentiometric surface 
Table 3. - Computation of vertical travel time and radius of well protection zones for wells in the leaky confined area-Continued

\begin{tabular}{|c|c|c|c|c|c|c|c|c|c|c|c|c|c|c|}
\hline We11 & $\begin{array}{l}\text { Lsd } \\
\text { (sea } \\
\text { lev.) }\end{array}$ & $\begin{array}{l}\text { Fi } \\
(s \in a \\
\text { lev.) }\end{array}$ & ${\underset{(f t)}{(t)}}^{m}$ & $\begin{array}{c}T \\
\left(f t^{2} / d\right)\end{array}$ & $\begin{array}{c}h \\
(\boldsymbol{e t})\end{array}$ & $\begin{array}{l}\text { WI } \\
\text { (sea } \\
\text { lev, }\end{array}$ & $\begin{array}{l}\text { Pot } \\
(s e a \\
\text { lev. }\end{array}$ & $(f t)$ & $\begin{array}{l}s(x) \\
(f t)\end{array}$ & $\begin{array}{l}\Delta \mathbf{h} \\
\text { (ft) }\end{array}$ & $\begin{array}{c}l \\
(f t)\end{array}$ & $\begin{array}{c}\text { Leak } \\
\text { (ft/d/ft) }\end{array}$ & $\begin{array}{c}t_{\mathbf{v}} \\
\text { (days) }\end{array}$ & $\begin{array}{r}r \\
(t)\end{array}$ \\
\hline & & \multicolumn{13}{|c|}{ Hi1lsborough County--Continued } \\
\hline $\begin{array}{l}01500 \\
01510 \\
01511 \\
01512 \\
01513 \\
01515 \\
01516 \\
01517 \\
01519 \\
01521\end{array}$ & $\begin{array}{l}53 \\
37 \\
43 \\
37 \\
43 \\
15 \\
16 \\
19 \\
23 \\
45\end{array}$ & $\begin{array}{r}0 \\
0 \\
0 \\
0 \\
0 \\
-10 \\
-10 \\
-10 \\
-10 \\
0\end{array}$ & $\begin{array}{l}1,080 \\
1,120 \\
1,120 \\
1,110 \\
1,120 \\
1,130 \\
1,130 \\
1,130 \\
1,130 \\
1,080\end{array}$ & $\begin{array}{l}26,000 \\
52,000 \\
52,000 \\
52,000 \\
52,000 \\
57,000 \\
57,000 \\
57,000 \\
57,000 \\
57,000\end{array}$ & $\begin{array}{r}E R R \\
502 \\
469 \\
453 \\
509 \\
69 \\
148 \\
151 \\
267 \\
405\end{array}$ & $\begin{array}{r}43 \\
30 \\
30 \\
30 \\
30 \\
7 \\
9 \\
12 \\
15 \\
40\end{array}$ & $\begin{array}{l}34 \\
20 \\
20 \\
20 \\
20 \\
11 \\
11 \\
12 \\
13 \\
25\end{array}$ & $\begin{array}{l}1.33 \\
0.69 \\
1.14 \\
0.71 \\
1.16 \\
0.58 \\
0.86 \\
1.17 \\
1.31 \\
1.52\end{array}$ & $\begin{array}{l}1 \\
2 \\
2 \\
2 \\
7 \\
5 \\
7 \\
5 \\
4\end{array}$ & $\begin{array}{r}11 \\
12 \\
12 \\
12 \\
3 \\
3 \\
7 \\
7 \\
19\end{array}$ & $\begin{array}{l}43 \\
30 \\
30 \\
30 \\
30 \\
17 \\
19 \\
22 \\
25 \\
40\end{array}$ & $\begin{array}{l}0.000360 \\
0.000300 \\
0.000300 \\
0.000300 \\
0.000300 \\
0.000300 \\
0.000300 \\
0.000300 \\
0.000300 \\
0.000300\end{array}$ & $\begin{array}{l}1,250 \\
1,176 \\
1,176 \\
1,176 \\
1,417 \\
1,583 \\
1,222 \\
1,389 \\
1,111\end{array}$ & $\begin{array}{r}720 \\
960 \\
760 \\
920 \\
1,840 \\
1,520 \\
1,760 \\
1,450 \\
1,240\end{array}$ \\
\hline $\begin{array}{l}01522 \\
01523 \\
01524 \\
01525 \\
01526 \\
01527 \\
01528 \\
01529 \\
01530 \\
01531\end{array}$ & $\begin{array}{l}47 \\
43 \\
60 \\
53 \\
53 \\
44 \\
51 \\
52 \\
51 \\
51\end{array}$ & $\begin{array}{l}0 \\
0 \\
0 \\
0 \\
0 \\
0 \\
0 \\
0 \\
0 \\
0\end{array}$ & $\begin{array}{l}1,080 \\
1,080 \\
1,060 \\
1,040 \\
1,040 \\
1,090 \\
1,100 \\
1,050 \\
1,050 \\
1,050\end{array}$ & $\begin{array}{l}57,000 \\
57,000 \\
52,000 \\
57,000 \\
57,000 \\
39,000 \\
39,000 \\
52,000 \\
52,000 \\
52,000\end{array}$ & $\begin{array}{l}353 \\
482 \\
470 \\
347 \\
447 \\
460 \\
456 \\
948 \\
499 \\
519\end{array}$ & $\begin{array}{l}40 \\
40 \\
49 \\
48 \\
48 \\
41 \\
40 \\
46 \\
46 \\
46\end{array}$ & $\begin{array}{l}25 \\
25 \\
32 \\
31 \\
31 \\
28 \\
29 \\
29 \\
29 \\
29\end{array}$ & $\begin{array}{l}1.52 \\
1.52 \\
1.32 \\
1.21 \\
1.21 \\
3.50 \\
3.50 \\
2.43 \\
2.43 \\
2.47\end{array}$ & $\begin{array}{l}4 \\
3 \\
3 \\
3 \\
3 \\
7 \\
7 \\
3 \\
5 \\
5\end{array}$ & $\begin{array}{l}19 \\
18 \\
20 \\
20 \\
20 \\
20 \\
18 \\
20 \\
22 \\
22\end{array}$ & $\begin{array}{l}40 \\
40 \\
49 \\
48 \\
48 \\
41 \\
40 \\
46 \\
46 \\
46\end{array}$ & $\begin{array}{l}0.000300 \\
0.000300 \\
0.000360 \\
0.000300 \\
0.000300 \\
0.000300 \\
0.000300 \\
0.000300 \\
0.000300 \\
0.000300\end{array}$ & $\begin{array}{l}1,111 \\
1,159 \\
1,089 \\
1,280 \\
1,280 \\
1,093 \\
1,159 \\
1,227 \\
1,136 \\
1,136\end{array}$ & $\begin{array}{r}1,330 \\
1,140 \\
1,020 \\
1,190 \\
1,050 \\
1,510 \\
1,510 \\
990 \\
1,360 \\
1,330\end{array}$ \\
\hline $\begin{array}{l}01532 \\
01533 \\
01534 \\
01536 \\
01537 \\
01538 \\
01539 \\
01540 \\
01541\end{array}$ & $\begin{array}{l}55 \\
54 \\
24 \\
43 \\
30 \\
37 \\
35 \\
40 \\
57\end{array}$ & $\begin{array}{l}0 \\
0 \\
0 \\
0 \\
0 \\
0 \\
0 \\
0 \\
0\end{array}$ & $\begin{array}{l}1,040 \\
1,040 \\
1,090 \\
1,080 \\
1,090 \\
1,100 \\
1,100 \\
1,100 \\
1,020\end{array}$ & $\begin{array}{l}52,000 \\
39,000 \\
57,000 \\
57,000 \\
57,000 \\
52,000 \\
57,000 \\
52,000 \\
57,000\end{array}$ & $\begin{array}{l}519 \\
496 \\
276 \\
607 \\
620 \\
613 \\
615 \\
610 \\
593\end{array}$ & $\begin{array}{l}50 \\
50 \\
20 \\
27 \\
25 \\
28 \\
28 \\
32 \\
51\end{array}$ & $\begin{array}{l}41 \\
43 \\
19 \\
21 \\
23 \\
23 \\
22 \\
22 \\
36\end{array}$ & $\begin{array}{l}2.25 \\
2.95 \\
7.27 \\
6.97 \\
6.97 \\
7.60 \\
6.97 \\
7.60 \\
6.97\end{array}$ & $\begin{array}{r}4 \\
6 \\
23 \\
11 \\
11 \\
12 \\
11 \\
12 \\
11\end{array}$ & $\begin{array}{l}13 \\
13 \\
24 \\
17 \\
13 \\
17 \\
17 \\
22 \\
26\end{array}$ & $\begin{array}{l}50 \\
50 \\
20 \\
27 \\
25 \\
28 \\
28 \\
32 \\
51\end{array}$ & $\begin{array}{l}0.000360 \\
0.000760 \\
0.000300 \\
0.000300 \\
0.000300 \\
0.000300 \\
0.000300 \\
0.000300 \\
0.000360\end{array}$ & $\begin{array}{r}1,543 \\
731 \\
460 \\
818 \\
926 \\
848 \\
848 \\
790 \\
914\end{array}$ & $\begin{array}{l}1,270 \\
1,300 \\
3,350 \\
2,260 \\
2,240 \\
2,250 \\
2,250 \\
2,260 \\
2,290\end{array}$ \\
\hline $\begin{array}{l}01609 \\
01681 \\
01682 \\
01754 \\
01793 \\
01794 \\
01795 \\
01826\end{array}$ & $\begin{array}{l}24 \\
67 \\
65 \\
22 \\
64 \\
63 \\
65 \\
21\end{array}$ & $\begin{array}{r}-10 \\
0 \\
0 \\
-180 \\
0 \\
0 \\
0 \\
-190\end{array}$ & $\begin{array}{l}1,040 \\
1,000 \\
1,000 \\
1,190 \\
1,150 \\
1,150 \\
1,150 \\
1,190\end{array}$ & $\begin{array}{r}57,000 \\
26,000 \\
26,000 \\
130,000 \\
200,000 \\
200,000 \\
200,000 \\
130,000\end{array}$ & $\begin{array}{l}146 \\
208 \\
210 \\
201 \\
213 \\
187 \\
210 \\
189\end{array}$ & $\begin{array}{r}20 \\
61 \\
61 \\
5 \\
28 \\
28 \\
28 \\
5\end{array}$ & $\begin{array}{r}17 \\
53 \\
53 \\
3 \\
15 \\
15 \\
15 \\
-1\end{array}$ & $\begin{array}{l}0.44 \\
0.87 \\
0.87 \\
0.25 \\
0.18 \\
0.18 \\
0.18 \\
0.18\end{array}$ & $\begin{array}{l}3 \\
4 \\
3 \\
1 \\
1 \\
1 \\
1 \\
1\end{array}$ & $\begin{array}{r}6 \\
12 \\
11 \\
3 \\
14 \\
14 \\
14 \\
7\end{array}$ & $\begin{array}{r}30 \\
61 \\
61 \\
185 \\
28 \\
28 \\
28 \\
195\end{array}$ & $\begin{array}{l}0.000300 \\
0.000760 \\
0.000760 \\
0.000089 \\
0.000067 \\
0.000067 \\
0.000067 \\
0.000013\end{array}$ & $\begin{array}{r}1,818 \\
944 \\
1,003 \\
51,966 \\
4,399 \\
4,399 \\
4,399 \\
250,000\end{array}$ & $\begin{array}{r}1,060 \\
890 \\
890\end{array}$ \\
\hline
\end{tabular}

and water-table maps, in order to compensate for the approximate nature of the maps.

The head difference, $\Delta l$, and other hydraulic parameters previously determined were then used to determine vertical travel times using equation 1 . For each well having a vertical travel time of 5 years or less, the radius of the required protection zone was calculated. Radii of the protection zones for these wells are listed in table 3.

\section{Calculation of Protection Zones for Individual Wells}

The protection zone around an individual well is a circle with the center of the circle at the well. The radius of the protection zone for each individual public-supply well with an average permitted withdrawal rate of 100,000 gallons or greater per day, or a well that is part of a well field with an average permitted withdrawal rate of 100,000 gallons or greater per day, was computed using the FDER formula
(Florida Department of Environmental Regulation, 1987):

$$
r=\left(\frac{Q t h}{\pi h n}\right)^{\frac{1}{2}},
$$

where

$\mathbf{r}=$ radius of individual zone of protection;

$t_{h}=$ horizontal travel time to well -5 years $(1,825$ days);

$\mathbf{n}=$ effective porosity of the Upper Floridan aquifer $=0.05$, as specified in the FDER regulation;

$\pi=3.14$;

$\mathbf{Q}=$ average permitted withdrawal rate for the well, im cubic feet per day; and

$h=$ average thickness of the Upper Floridan aquifer penetrated by the well, im feet. 
The thickness of the Upper Floridan aquifer interval penetrated was determined for unconfined areas by subtracting the depth to water in the Upper Floridan aquifer from the depth of the well. The depth of the well was obtained from SWFWMD files. The depth to water was determined using a multicounty water-level altitude map with a contour interval of 10 feet (Barr, 1985a) and land-surface altitudes from topographic maps having 5- to 10-foot contour intervals. In areas where the Floridan aquifer is leaky confined, the thickness was determined by subtracting the depth to the top of the Floridan from the well depth. The depth to the top of the Floridan was determined either by using maps of the top of the aquifer (Stewart, 1968; Buono and Rutledge, 1979) or from well-field reports (Ryder, 1978; Ryder and others, 1980; Hutchinson, 1985) and land surface from topographic maps. The permitted withdrawal rates were obtained from SWFWMD permit files dated February 2,1987 . In all cases, map data were derived from the locations determined from SWFWMD latitude-longitude coordinates for each public-supply well.

The result of this calculation is the radius of a circle around each permitted well that describes the zone of protection for that well. The computed protection zone radii and the data used in the calculations for the unconfined areas of Citrus, Hernando, and Pasco Counties are shown in table 4. Similar information for individual wells included in the major well fields in leaky confined areas with vertical travel times of less than 5 years is shown in table 5 . The tabulated radius and location may be used to delineate the protection zone on a map of any scale.

\section{Calculation of Protection Zones for Well Fields}

The circles delineating individual protection zones overlap in some cases. Because the formula is based on a volumetric displacement concept, overlapping protection zones underestimate the amount of protection required by an amount equal to the area of the overlap.

The procedure used to determine zones of protection around individual wells with overlapping zones was to add the areas of the overlapping zones together to determine a composite protection-zone area. In some cases, groups of wells were permitted together at a withdrawal rate less than the sum of the permitted rates of the individual wells. Such groups of wells with overlapping zones were treated the same as well fields. For well fields, a composite area for the protection zone was determined using a formula analogous to that used to calculate the radius of protection:

$$
A=\frac{Q t_{h}}{h n},
$$

where

$A=$ area of well field zone of protection, in square feet;

$t_{h}=$ horizontal travel time to well -5 years $(1,825$ days);

$\mathbf{n}=$ effective porosity of the Upper Floridan aquifer $=0.05$, as specified in the FDER regulation;

$\mathbf{Q}=$ average permitted withdrawal rate for the well field, in cubic feet per day; and

h = average thickness of the Upper Floridan aquifer penetrated in the well field, in feet.

These areas were delineated on a map of each well field, or on a quadrangle sheet for individual wells with overlapping zones, in a shape that approximated the configuration of wells in the field. This was done by successive approximation until the designated area was within 5 percent of the calculated area. In all cases, the boundary of the composite protection zone was equal to or outside the boundary of the protection zone for each individual well. If a composite protection zone overlapped protection zones of additional wells, those areas were added to the total and the zone was recomputed.

Well fields with composite protection zones are designated in table 6. Selected well field zones are individually mapped in plates 1-3. Individual wells with overlapping zones, thus requiring composite zones, are noted in table 7 and selected ones are mapped in figures 7-9. Maps showing composite protection zones for other wells or well fields are on file with FDER.

This method of delineating composite protection zones, as with any procedure, creates some anomalous situations. A good example is the Morris Bridge well field (pl.3). The permitted well field pumping rate for Morris Bridge of $15.5 \mathrm{Mgal} / \mathrm{d}$ is not only considerably less than the sum of the permitted rates for the individual wells in the field (21.9 Mgal/d), but yields an area less than the area formed by the outside boundary of the intersecting circles which is by rule the boundary of the protection zone (pl. 3). This zone of protection, however, does not include some area in the center of the well field which would seem to be logically included because water cannot flow there except through the zone of protection. An alternative composite zone of protection was delineated for illustrative purposes by assigning the calculated area outside of a perimeter formed by connecting all of the outermost wells in the field. This zone is more conservative and includes all of the interior areas excluded by the rule method. 
Table 4. - Computation of well protection zones in the unconfined area

Sources and explanation of data for calculations :

Well - Well number from Southwest Florida Water Management District (SWFWM) files. Blank where unavailable.

Lat. Long. Sec. Twn, and Rng - Latitude, longitude, section, township, and range. From SwFwid permitting files, February 2, 1987 .

Cons, use permit - Consumptive use permit number. From SWFWD permitting files, February 2 , 1987.

Av. Q - Permitted average pumping rate, in gallons per day, for public-supply use. From SWFWM permitting files, February 2, 1987 .

Well depth, in feet - From SWFWM files, with some additions from U.S. Geological Survey data files. Blank where unavailable.

Well name - From SWFWM files. Blank where unavailable.

Owner - From SWFWM permitting files, February 2, 1987.

County - From SWFWMD permitting files, February 2, 1987.

Lsd - Altitude of land surface, in feet above sea leve1, from U.S. Geological Survey $7 \frac{1}{2}-m i n u t e$ topographic maps at latitude and longitude given.

F1 - Top of saturated zone in Upper Floridan aquifer, May 1985 (Barr, 1985a), in feet above sea level.

$\underline{\underline{h}}$ - Saturated thickness of Upper Floridan aquifer penetrated by the well, in feet. (Well depth - [Lsd-F1]).

$\underline{\underline{r}}$ - Radius of protection zone of the well, rounded to the nearest 10 feet. $\left.r=\left[\frac{18250}{7.48 \pi} \mathrm{hn}\right]\right]^{\frac{1}{2}} \mathrm{n}=0.05$ for the Upper Floridan aquifer. Radius not computed where well depth unavailable.

\begin{tabular}{|c|c|c|c|c|c|c|c|c|c|c|c|}
\hline Well & lat. & Long. & Sec. & $\begin{array}{l}\text { Twn; } \\
\text { (S.) }\end{array}$ & $\begin{array}{c}\text { Cons } \\
\text { use } \\
\text { permit }\end{array}$ & $\begin{array}{c}\text { Av.Q } \\
(\mathrm{Bal} / \mathrm{d})\end{array}$ & $\begin{array}{l}\text { Well } \\
\text { depth Well } \\
\text { (ft) name }\end{array}$ & Owner & $\begin{array}{l}\text { Lsd } \\
\text { (sea } \\
\text { lev.) }\end{array}$ & $\begin{array}{l}\text { F1 } \\
\text { (sea } \\
\text { lev.) }\end{array}$ & $\stackrel{h}{\text { (ft) }}$ \\
\hline
\end{tabular}

Citrus County

\begin{tabular}{|c|c|c|c|c|c|c|c|c|c|c|c|c|c|c|c|}
\hline $\begin{array}{l}00050 \\
00051 \\
00052 \\
00102 \\
00103 \\
00104 \\
00194 \\
00195\end{array}$ & $\begin{array}{l}285356 \\
285406 \\
285404 \\
285022 \\
285022 \\
284925 \\
284437 \\
284650\end{array}$ & $\begin{array}{l}823530 \\
823438 \\
823438 \\
822006 \\
822006 \\
822046 \\
821754 \\
821747\end{array}$ & $\begin{array}{r}21 \\
22 \\
22 \\
17 \\
17 \\
19 \\
15 \\
3\end{array}$ & $\begin{array}{l}18 \\
18 \\
18 \\
19 \\
19 \\
19 \\
20 \\
20\end{array}$ & $\begin{array}{l}17 \\
17 \\
17 \\
20 \\
20 \\
20 \\
20 \\
20\end{array}$ & $\begin{array}{l}0020702 \\
0020702 \\
0020702 \\
0041902 \\
0041902 \\
0041902 \\
0111801 \\
0111801\end{array}$ & $\begin{array}{r}250,000 \\
600,000 \\
250,000 \\
600,000 \\
600,000 \\
1,000,000 \\
194,000 \\
194,000\end{array}$ & $\begin{array}{l}157 \\
250 \\
175 \\
400 \\
250 \\
250 \\
193 \\
195\end{array}$ & $\begin{array}{r}1 \\
\mathrm{P}-1 \\
\mathrm{P}-2 \\
1 \\
2 \\
3 \\
2 \\
3 \mathrm{P}\end{array}$ & $\begin{array}{l}\text { FLORAL } \\
\text { FLORAL }\end{array}$ & $\begin{array}{r}\text { CRYSTAL RIVER, CITY OF } \\
\text { CRYSTAL RIVER, CITY OF } \\
\text { CRYSTAL RIVER, CITY OF } \\
\text { INVERNESS, CITY OF } \\
\text { INVERNESS, CITY OF } \\
\text { INVERNESS, CITY OF } \\
\text { CITY WATER ASSOC INC. } \\
\text { CITY WATER ASSOC INC. }\end{array}$ & $\begin{array}{r}5 \\
7 \\
7 \\
58 \\
58 \\
70 \\
82 \\
49\end{array}$ & $\begin{array}{r}1 \\
2 \\
2 \\
30 \\
30 \\
20 \\
30 \\
30\end{array}$ & $\begin{array}{l}153 \\
245 \\
170 \\
372 \\
222 \\
200 \\
141 \\
176\end{array}$ & $\begin{array}{l}1,590 \\
1,950 \\
1,510 \\
1,580 \\
2,050 \\
2,790 \\
1,460 \\
1,310\end{array}$ \\
\hline $\begin{array}{l}00411 \\
00416 \\
00599 \\
00598\end{array}$ & $\begin{array}{l}290033 \\
285754 \\
284429 \\
284421 \\
284223 \\
284212 \\
284450 \\
284412\end{array}$ & $\begin{array}{l}822730 \\
822849 \\
823101 \\
823101 \\
823125 \\
823115 \\
823058 \\
823055\end{array}$ & $\begin{array}{l}11 \\
27 \\
16 \\
16 \\
32 \\
32 \\
16 \\
21\end{array}$ & $\begin{array}{l}17 \\
17 \\
20 \\
20 \\
20 \\
20 \\
20 \\
20\end{array}$ & $\begin{array}{l}18 \\
18 \\
18 \\
18 \\
18 \\
18 \\
18 \\
18\end{array}$ & $\begin{array}{l}0284202 \\
0284202 \\
0367302 \\
0367302 \\
0367302 \\
0367302 \\
0367302 \\
0367302\end{array}$ & $\begin{array}{l}252,000 \\
170,000 \\
432,000 \\
432,000 \\
432,000 \\
432,000 \\
432,000 \\
432,000\end{array}$ & $\begin{array}{l}250 \\
312 \\
325 \\
325 \\
325 \\
325\end{array}$ & $\begin{array}{r}\text { CS7 } \\
13 \\
5 \\
6 \\
8 \\
9 \\
10 \\
11\end{array}$ & CI? & $\begin{array}{l}\text { TRUS SPRINGS UTILITIES } \\
\text { TRUS SPRINGS UTILITIES } \\
\text { PUNTA GORDA DEVELOPERS } \\
\text { PUNTA GORDA DEVELOPERS } \\
\text { PUNTA GORDA DEVELOPERS } \\
\text { PUNTA GORDA DEVELOPERS } \\
\text { PUNTA GORDA DEVELOPERS } \\
\text { PUNTA GORDA DEVELOPERS }\end{array}$ & $\begin{array}{r}105 \\
84 \\
91 \\
88 \\
60 \\
72 \\
100 \\
88\end{array}$ & $\begin{array}{r}10 \\
5 \\
6 \\
6 \\
7 \\
7 \\
6 \\
7\end{array}$ & $\begin{array}{r}89 \\
\\
165 \\
230 \\
272 \\
260 \\
231 \\
244\end{array}$ & $\begin{array}{l}2,020 \\
1,710 \\
1,570 \\
1,610 \\
1,700 \\
1,660\end{array}$ \\
\hline $\begin{array}{l}00648 \\
00649 \\
00650 \\
00651\end{array}$ & $\begin{array}{l}284407 \\
284453 \\
284230 \\
284234 \\
285532 \\
285532 \\
285503 \\
285446\end{array}$ & $\begin{array}{l}823057 \\
823058 \\
823134 \\
823144 \\
822709 \\
822709 \\
822700 \\
822718\end{array}$ & $\begin{array}{l}21 \\
16 \\
32 \\
29 \\
11 \\
11 \\
13 \\
14\end{array}$ & $\begin{array}{l}20 \\
20 \\
20 \\
20 \\
18 \\
18 \\
18 \\
18\end{array}$ & $\begin{array}{l}18 \\
18 \\
18 \\
18 \\
18 \\
18 \\
18 \\
18\end{array}$ & $\begin{array}{l}0367302 \\
0367302 \\
0367302 \\
0367302 \\
0415302 \\
0415302 \\
0415302 \\
0415302\end{array}$ & $\begin{array}{l}432,000 \\
432,000 \\
432,000 \\
432,000 \\
215,000 \\
175,000 \\
130,000 \\
215,000\end{array}$ & $\begin{array}{l}300 \\
300 \\
300 \\
300 \\
320 \\
350 \\
280 \\
280\end{array}$ & $\begin{array}{r}12 \\
13 \\
14 \\
15 \\
1 \\
2 \\
4 \\
5\end{array}$ & $\begin{array}{l}\text { ROLLII } \\
\text { ROLLII } \\
\text { ROLLII } \\
\text { ROLLII }\end{array}$ & $\begin{array}{l}\text { PUNTA GORDA DEVELOPERS } \\
\text { PUNTA GORDA DEVELOPERS } \\
\text { PUNTA GORDA DEVELOPERS } \\
\text { PUNTA GORDA DEVELOPERS } \\
\text { NG OAKS UTILITIES INC. } \\
\text { NG OAKS UTILITIES INC. } \\
\text { NG OAKS UTILITIES INC. } \\
\text { NG OAKS UTILITIES INC. }\end{array}$ & $\begin{array}{r}70 \\
110 \\
61 \\
60 \\
132 \\
132 \\
70 \\
79\end{array}$ & $\begin{array}{l}7 \\
6 \\
7 \\
7 \\
4 \\
4 \\
5 \\
4\end{array}$ & $\begin{array}{l}237 \\
196 \\
246 \\
247 \\
192 \\
222 \\
215 \\
205\end{array}$ & $\begin{array}{l}1,680 \\
1,850 \\
1,650 \\
1,650 \\
1,320 \\
1,110 \\
970 \\
1,280\end{array}$ \\
\hline $\begin{array}{l}00652 \\
00653 \\
00654 \\
00655 \\
00872 \\
00873 \\
00874\end{array}$ & $\begin{array}{l}285513 \\
285447 \\
285458 \\
285432 \\
285458 \\
284720 \\
284736 \\
284820\end{array}$ & $\begin{array}{l}822758 \\
822803 \\
822813 \\
822737 \\
822616 \\
823455 \\
823429 \\
823215\end{array}$ & $\begin{array}{l}14 \\
14 \\
15 \\
14 \\
13 \\
27 \\
27 \\
24\end{array}$ & $\begin{array}{l}18 \\
18 \\
18 \\
18 \\
18 \\
19 \\
19 \\
19\end{array}$ & $\begin{array}{l}18 \\
18 \\
18 \\
18 \\
18 \\
17 \\
17 \\
17\end{array}$ & $\begin{array}{l}0415302 \\
0415302 \\
0415302 \\
0415302 \\
0415302 \\
0440603 \\
0440603 \\
0440603\end{array}$ & $\begin{array}{l}215,000 \\
259,000 \\
345,000 \\
518,000 \\
518,000 \\
138,000 \\
138,000 \\
264,000\end{array}$ & $\begin{array}{l}340 \\
300 \\
405 \\
355 \\
\\
110 \\
195 \\
190\end{array}$ & $\begin{array}{r}6 \\
7 \\
8 \\
9 \\
10 \\
1 \\
3 \mathrm{BRA} \\
4 \mathrm{NOR}\end{array}$ & $\begin{array}{l}\text { ROLLII } \\
\text { ROLLII } \\
\text { ROLLII } \\
\text { ROLLII } \\
\text { ROLLI } \\
\text { HOMOSASS } \\
\text { HOMOSASS } \\
\text { HOMOSASS }\end{array}$ & $\begin{array}{l}\text { NG OAKS UTILITIES INC. } \\
\text { NG OAKS UTILITIES INC. } \\
\text { NG OAKS UTILITIES INC. } \\
\text { NG OAKS UTILITIES INC. } \\
\text { ING OAKS UTILITIES INC. } \\
\text { A SPECIAL WATER DISTRI } \\
\text { AA SPECIAL WATER DISTRI } \\
\text { SA SPECIAL WATER DISTRI }\end{array}$ & $\begin{array}{r}96 \\
104 \\
106 \\
126 \\
96 \\
7 \\
9 \\
15\end{array}$ & $\begin{array}{l}4 \\
4 \\
4 \\
4 \\
5 \\
2 \\
2 \\
3\end{array}$ & $\begin{array}{l}248 \\
200 \\
303 \\
233 \\
\\
\\
105 \\
188 \\
178\end{array}$ & $\begin{array}{l}1,160 \\
1,420 \\
1,330 \\
1,860 \\
1,430 \\
1,070 \\
1,520\end{array}$ \\
\hline $\begin{array}{l}00875 \\
00876 \\
00961 \\
01641 \\
01642 \\
01692 \\
01693 \\
01821\end{array}$ & $\begin{array}{l}284720 \\
284809 \\
285200 \\
285323 \\
285320 \\
285330 \\
285330 \\
284103\end{array}$ & $\begin{array}{l}823453 \\
823055 \\
823425 \\
823158 \\
823158 \\
822640 \\
822638 \\
821841\end{array}$ & $\begin{array}{r}27 \\
29 \\
34 \\
30 \\
30 \\
25 \\
25 \\
4\end{array}$ & $\begin{array}{l}19 \\
19 \\
18 \\
18 \\
18 \\
18 \\
18 \\
21\end{array}$ & $\begin{array}{l}17 \\
18 \\
17 \\
18 \\
18 \\
18 \\
18 \\
20\end{array}$ & $\begin{array}{l}0440603 \\
0440603 \\
0465901 \\
0712100 \\
0712100 \\
0735800 \\
0735800 \\
0787900\end{array}$ & $\begin{array}{l}100,000 \\
396,000 \\
400,000 \\
167,000 \\
167,000 \\
393,000 \\
393,000 \\
112,000\end{array}$ & $\begin{array}{r}110 \\
333 \\
90 \\
\\
\\
350 \\
350 \\
210\end{array}$ & $\begin{array}{l}2 \\
5 \\
3 \\
1 \\
2 \\
1 \\
2 \\
1\end{array}$ & $\begin{array}{r}\text { HOMOSASS } \\
\text { HOMOSASS } \\
\text { OZELLO } \\
\text { MEADC } \\
\text { MEAD } \\
\text { SOUTHERN }\end{array}$ & $\begin{array}{l}\text { A SPECIAL WATER DISTRI } \\
\text { SA SPECIAL WATER DISTRI } \\
\text { WATER ASSOCIATION INC. } \\
\text { OWCREST UTILITIES INC. } \\
\text { OWCREST UTILITIES INC. } \\
\text { CITRUS COUNTY } \\
\text { CITRUS COUNTY } \\
\text { STATES UTILITIES INC. }\end{array}$ & $\begin{array}{r}7 \\
132 \\
10 \\
70 \\
70 \\
209 \\
208 \\
89\end{array}$ & $\begin{array}{r}2 \\
4 \\
2 \\
3 \\
3 \\
5 \\
5 \\
29\end{array}$ & $\begin{array}{r}105 \\
205 \\
82\end{array}$ & $\begin{array}{l}1,220 \\
1,730 \\
2,750\end{array}$ \\
\hline 01822 & $\begin{array}{l}284103 \\
290013 \\
290013\end{array}$ & $\begin{array}{l}821840 \\
824005 \\
824005\end{array}$ & $\begin{array}{r}4 \\
11 \\
11\end{array}$ & $\begin{array}{l}21 \\
17 \\
17\end{array}$ & $\begin{array}{l}20 \\
16 \\
16\end{array}$ & $\begin{array}{l}0787900 \\
0862300 \\
0862300\end{array}$ & $\begin{array}{l}112,000 \\
120,000 \\
120,000\end{array}$ & $\begin{array}{l}210 \\
100 \\
100\end{array}$ & 2 & SOUTHERN & $\begin{array}{l}\text { STATES UTILITIES INC. } \\
\text { CITRUS CONDOS, INC. } \\
\text { CITRUS CONDOS, INC. }\end{array}$ & $\begin{array}{l}89 \\
17 \\
17\end{array}$ & $\begin{array}{r}29 \\
5 \\
5\end{array}$ & $\begin{array}{r}150 \\
88 \\
88\end{array}$ & $\begin{array}{l}1,080 \\
1,460 \\
1,460\end{array}$ \\
\hline \multicolumn{16}{|c|}{ Hernando County } \\
\hline $\begin{array}{l}00095 \\
00227 \\
00228 \\
00505 \\
00989\end{array}$ & $\begin{array}{l}283227 \\
283130 \\
283130 \\
283241 \\
283222\end{array}$ & $\begin{array}{l}823400 \\
821348 \\
821400 \\
823121 \\
823235\end{array}$ & $\begin{array}{l}26 \\
32 \\
32 \\
29 \\
30\end{array}$ & $\begin{array}{l}22 \\
22 \\
22 \\
22 \\
22\end{array}$ & $\begin{array}{l}17 \\
21 \\
21 \\
18 \\
18\end{array}$ & $\begin{array}{l}0036801 \\
0165101 \\
0165101 \\
0298302 \\
0298302\end{array}$ & $\begin{array}{l}290,000 \\
300,000 \\
150,000 \\
300,000 \\
335,000\end{array}$ & $\begin{array}{l}245 \\
600 \\
275 \\
500 \\
320\end{array}$ & $\begin{array}{r}1 \\
1 \\
2 \\
3 \\
\text { WH-1 }\end{array}$ & ROYAL & $\begin{array}{l}\text { PALM BEACH COLONY INC. } \\
\text { HERNANDO COUNTY } \\
\text { HERNANDO COUNTY } \\
\text { HERNANDO COUNTY } \\
\text { HERNANDO COUNTY }\end{array}$ & $\begin{array}{l}21 \\
71 \\
90 \\
65 \\
35\end{array}$ & $\begin{array}{l}11 \\
45 \\
45 \\
17 \\
15\end{array}$ & $\begin{array}{l}235 \\
574 \\
230 \\
452 \\
300\end{array}$ & $\begin{array}{r}1,380 \\
900 \\
1,010 \\
1,020 \\
1,320\end{array}$ \\
\hline
\end{tabular}


Table 4. - Computation of well protection zones in the unconfined area-Continued

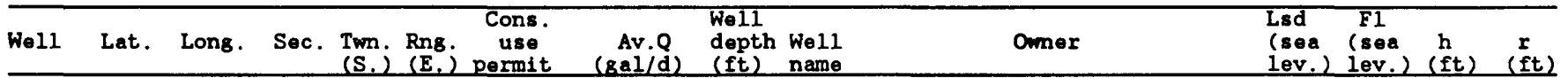

\begin{tabular}{|c|c|c|c|c|c|c|c|c|c|}
\hline $\begin{array}{l}00990 \\
00991 \\
00992 \\
01586 \\
01587 \\
00536 \\
00537 \\
01085 \\
01086\end{array}$ & $\begin{array}{l}283235 \\
283245 \\
283302 \\
283244 \\
283305 \\
283126 \\
283133 \\
282724 \\
282652\end{array}$ & $\begin{array}{l}823233 \\
823230 \\
823225 \\
822933 \\
822930 \\
821736 \\
821736 \\
823633 \\
823637\end{array}$ & $\begin{array}{l}30 \\
30 \\
19 \\
27 \\
22 \\
34 \\
34 \\
28 \\
33\end{array}$ & $\begin{array}{l}22 \\
22 \\
22 \\
22 \\
22 \\
22 \\
22 \\
23 \\
23\end{array}$ & $\begin{array}{l}18 \\
18 \\
18 \\
18 \\
18 \\
20 \\
20 \\
17 \\
17\end{array}$ & $\begin{array}{l}0298302 \\
0298302 \\
0298302 \\
0298302 \\
0298302 \\
0340802 \\
0340802 \\
0484204 \\
0484204\end{array}$ & $\begin{array}{l}335,000 \\
335,000 \\
335,000 \\
275,000 \\
175,000 \\
121,000 \\
121,000 \\
227,000 \\
227,000\end{array}$ & $\begin{array}{l}218 \\
275 \\
275 \\
700 \\
325 \\
110 \\
400 \\
373 \\
286\end{array}$ & $\begin{array}{l}\text { WH-2 } \\
\text { WH-3 } \\
\text { WH-4 }\end{array}$ \\
\hline $\begin{array}{l}01087 \\
01088 \\
01089 \\
01090 \\
01091 \\
01092 \\
01093 \\
01094\end{array}$ & $\begin{array}{l}282751 \\
282937 \\
282726 \\
282708 \\
282934 \\
283042 \\
282817 \\
283044\end{array}$ & $\begin{array}{l}823039 \\
823317 \\
823638 \\
823351 \\
823318 \\
823104 \\
823306 \\
823106\end{array}$ & $\begin{array}{r}21 \\
12 \\
28 \\
25 \\
12 \\
4 \\
19 \\
4\end{array}$ & $\begin{array}{l}23 \\
23 \\
23 \\
23 \\
23 \\
23 \\
23 \\
23\end{array}$ & $\begin{array}{l}18 \\
17 \\
17 \\
17 \\
17 \\
18 \\
18 \\
18\end{array}$ & $\begin{array}{l}0484204 \\
0484204 \\
0484204 \\
0484204 \\
0484204 \\
0484204 \\
0484204 \\
0484204\end{array}$ & $\begin{array}{l}566,000 \\
397,000 \\
397,000 \\
227,000 \\
227,000 \\
453,000 \\
325,000 \\
325,000\end{array}$ & $\begin{array}{l}320 \\
418 \\
350 \\
395 \\
484 \\
400 \\
290 \\
400\end{array}$ & $\begin{array}{r}7 \\
10 \\
11 \\
12 \\
13 \\
17 \\
18 \\
19 P\end{array}$ \\
\hline $\begin{array}{l}01095 \\
01096 \\
01178\end{array}$ & $\begin{array}{l}282754 \\
282710 \\
282849 \\
282937 \\
283000 \\
283030 \\
282833 \\
282833\end{array}$ & $\begin{array}{l}823036 \\
823352 \\
822735 \\
822832 \\
821038 \\
821141 \\
823606 \\
823606\end{array}$ & $\begin{array}{r}21 \\
25 \\
13 \\
11 \\
11 \\
3 \\
21 \\
21\end{array}$ & $\begin{array}{l}23 \\
23 \\
23 \\
23 \\
23 \\
23 \\
23 \\
23\end{array}$ & $\begin{array}{l}18 \\
17 \\
18 \\
18 \\
21 \\
21 \\
17 \\
17\end{array}$ & $\begin{array}{l}0484204 \\
0484204 \\
0518602 \\
0575501 \\
0578901 \\
0578901 \\
0603901 \\
0603901\end{array}$ & $\begin{array}{l}325,000 \\
227,000 \\
112,000 \\
150,000 \\
184,000 \\
184,000 \\
450,000 \\
450,000\end{array}$ & $\begin{array}{l}300 \\
300 \\
500 \\
200 \\
360 \\
144 \\
200 \\
200\end{array}$ & $\begin{array}{r}20 P \\
21 P \\
9 P\end{array}$ \\
\hline $\begin{array}{l}01585 \\
01748 \\
01749 \\
01750 \\
01751 \\
01752 \\
01753\end{array}$ & $\begin{array}{l}283238 \\
283304 \\
283304 \\
283411 \\
283127 \\
283129 \\
283443\end{array}$ & $\begin{array}{l}823401 \\
822323 \\
822321 \\
822334 \\
822355 \\
822351 \\
822239\end{array}$ & $\begin{array}{l}26 \\
22 \\
22 \\
15 \\
34 \\
34 \\
11\end{array}$ & $\begin{array}{l}22 \\
22 \\
22 \\
22 \\
22 \\
22 \\
22\end{array}$ & $\begin{array}{l}17 \\
19 \\
19 \\
19 \\
19 \\
19 \\
19\end{array}$ & $\begin{array}{l}0689500 \\
0762700 \\
0762700 \\
0762700 \\
0762700 \\
0762700 \\
0762700\end{array}$ & $\begin{array}{l}200,000 \\
200,000 \\
200,000 \\
400,000 \\
250,000 \\
550,000 \\
200,000\end{array}$ & $\begin{array}{l}245 \\
593 \\
750 \\
740 \\
750 \\
600 \\
450\end{array}$ & $\begin{array}{l}1 \\
1 \\
2 \\
3 \\
4 \\
5 \\
6\end{array}$ \\
\hline
\end{tabular}

Pasco County

$\begin{array}{lllllll}00080 & 281818 & 824121 & 15 & 25 & 16 & 0027902\end{array}$

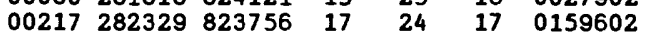

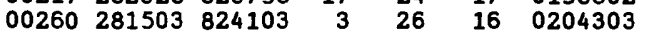

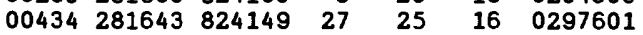

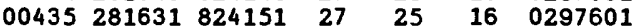

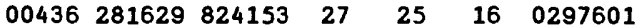
$\begin{array}{lllllll}00437 & 281627 & 824129 & 27 & 25 & 16 & 0297601\end{array}$

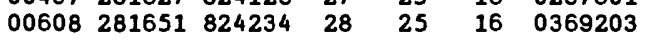

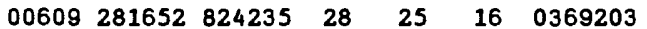
$\begin{array}{lllllll}00622 & 281852 & 824054 & 15 & 25 & 16 & 0375901\end{array}$ $\begin{array}{lllllll}00623 & 282042 & 823657 & 4 & 25 & 17 & 0376101\end{array}$

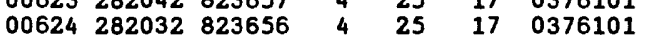

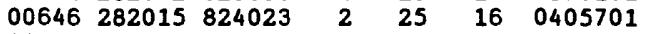

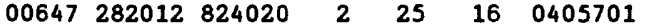

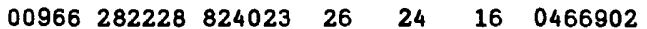

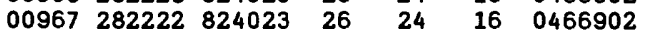

$\begin{array}{lllllll}00968 & 282218 & 824023 & 26 & 24 & 16 & 0466902\end{array}$ $\begin{array}{lllllll}00969 & 282226 & 824015 & 26 & 24 & 16 & 0466902\end{array}$ $\begin{array}{lllllll}00970 & 282222 & 824015 & 26 & 24 & 16 & 0466902\end{array}$

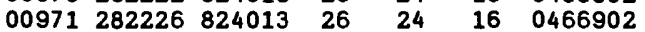
$\begin{array}{lllllll}00972 & 282223 & 824013 & 26 & 24 & 16 & 0466902\end{array}$

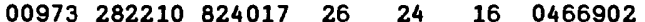

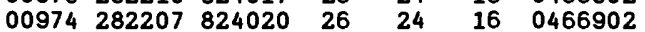

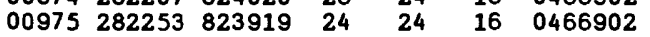

$\begin{array}{lllllll}00976 & 282253 & 823923 & 24 & 24 & 16 & 0466902\end{array}$

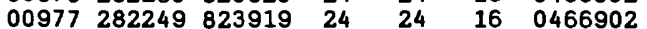

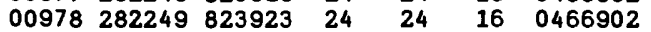

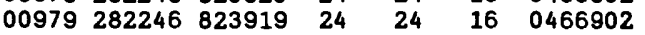
$\begin{array}{lllllll}00980 & 282246 & 823923 & 24 & 24 & 16 & 0466902\end{array}$

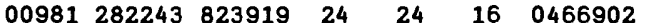

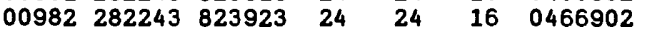

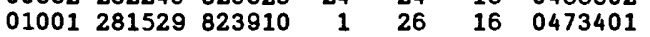

$\begin{array}{lllllll}01670 & 282055 & 823911 & 36 & 24 & 16 & 0728200\end{array}$

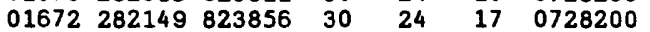

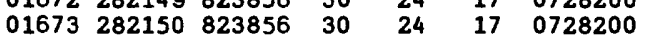

$100,000 \quad 60$ SEWG

$103,000 \quad 410 \quad 2$

$150,000 \quad 385$

150,000281

$150,000 \quad 267$

$150,000 \quad 209$

$200,000 \quad 200$

112,000230

$237,000 \quad 150$

$102,000 \quad 110$

$435,000 \quad 350$

$115,000 \quad 350$

125,000

100,000

$100,000 \quad 100$

$100,000 \quad 100$

$100,000 \quad 100$

$100,000 \quad 50$

$100,000 \quad 100$

$100,000 \quad 90$

$100,000 \quad 105$

$100,000 \quad 93 \quad 15$

$100,000 \quad 100 \quad 16$

$100,000 \quad 100 \quad 17$

$100,000 \quad 100 \quad 18$

$100,000 \quad 100 \quad 19$

$\begin{array}{lll}100,000 & 100 & 20 \\ 100,000 & 100 & 21\end{array}$

$100,000 \quad 100 \quad 21$

$100,000 \quad 100 \quad 22$

$\begin{array}{rrr}140,000 & 300 & w-5\end{array}$

$200,000 \quad 121 \quad$ PW-1

$375,000 \quad 133 \quad$ PW-3
$100,000 \quad 95 \quad 14$

375,000133 PW-4

Bernando County--Continued

$\begin{array}{rrrrr}\text { HERNANDO COUNTY } & 52 & 15 & 181 & 1,700 \\ \text { HERNANDO COUNTY } & 25 & 15 & 265 & 1,400 \\ \text { HERNANDO COUNTY } & 31 & 15 & 259 & 1,420 \\ \text { HERNANDO COUNTY } & 94 & 23 & 629 & 820 \\ \text { HERNANDO COUNTY } & 74 & 22 & 273 & 1,000 \\ \text { HERNANDO COUNTY } & 100 & 42 & 52 & 1,900 \\ \text { HERNANDO COUNTY } & 100 & 42 & 342 & 740 \\ \text { SPRING BILL UTILITIES } & 34 & 15 & 354 & 1,000 \\ \text { SPRING HILL UTILITIES } & 31 & 16 & 271 & 1,140 \\ & & & & \\ \text { SPRING HILL UTILITIES } & 60 & 25 & 285 & 1,760 \\ \text { SPRING HILL UTILITIES } & 35 & 16 & 399 & 1,240 \\ \text { SPRING HILL UTILITIES } & 34 & 15 & 331 & 1,360 \\ \text { SPRING HILL UTILITIES } & 35 & 20 & 380 & 960 \\ \text { SPRING HILL UTILITIES } & 35 & 16 & 465 & 870 \\ \text { SPRING HILL UTILITIES } & 82 & 22 & 340 & 1,440 \\ \text { SPRING HILL UTILITIES } & 39 & 21 & 272 & 1,360 \\ \text { SPRING BILL UTILITIES } & 82 & 22 & 340 & 1,220 \\ & & & & \\ \text { SPRING HILL UTILITIES } & 60 & 25 & 265 & 1,380 \\ \text { SPRING BILL UTILITIES } & 35 & 20 & 285 & 1,110 \\ \text { HERNANDO COUNTY } & 69 & 32 & 463 & 610 \\ \text { HERNANDO COUNTY } & 70 & 29 & 159 & 1,210 \\ \text { HERNANDO COUNTY } & 77 & 48 & 331 & 930 \\ \text { HERNANDO COUNTY } & 72 & 46 & 118 & 1,560 \\ \text { U S BOME CORP } & 36 & 14 & 178 & 1,980 \\ \text { U S BOME CORP } & 36 & 14 & 178 & 1,980 \\ \text { BROOKSVILLE, CITY OF } & 138 & 35 & 490 & 800 \\ \text { BROOKSVILLE, CITY OF } & 138 & 35 & 647 & 690 \\ \text { BROOKSVILLE, CITY OF } & 175 & 35 & 600 & 1,020 \\ \text { BROOKSVILLE, CITY OF } & 186 & 37 & 601 & 800 \\ \text { BROOKSVILLE, CITY OF } & 202 & 37 & 435 & 1,400 \\ \text { BROOKSVILLE, CITY OF } & 92 & 35 & 393 & 890\end{array}$

JASMINE LAKES SERVICES INC. MYRICK, GEORGE
ORANGEWOOD LAKES MOBILE HOME PASCO COUNTY PASCO COUNTY

PASCO COUNTY PASCO COUNTY

PORT RICHEY, CITY OF

PORT RICHEY, CITY OF

SMITH UTILITIES INC C I PASCO COUNTY PASCO COUNTY PASCO COUNTY PASCO COUNTY

HUDSON WATER WORKS INC. HUDSON WATER WORKS INC.

\begin{tabular}{|c|c|c|c|}
\hline $\begin{array}{l}11 \\
25 \\
20 \\
35 \\
19 \\
33 \\
25 \\
22\end{array}$ & $\begin{array}{r}3 \\
18 \\
5 \\
4 \\
4 \\
4 \\
4 \\
1\end{array}$ & $\begin{array}{r}52 \\
403 \\
370 \\
250 \\
252 \\
180 \\
179 \\
209\end{array}$ & $\begin{array}{r}1,730 \\
630 \\
790 \\
970 \\
960 \\
1,140 \\
1,320 \\
910\end{array}$ \\
\hline $\begin{array}{l}20 \\
21 \\
34 \\
36 \\
25 \\
30\end{array}$ & $\begin{array}{r}1 \\
2 \\
25 \\
25 \\
4 \\
4\end{array}$ & $\begin{array}{r}131 \\
91 \\
341 \\
339\end{array}$ & $\begin{array}{r}1,680 \\
1,320 \\
1,410 \\
730\end{array}$ \\
\hline $\begin{array}{l}21 \\
20\end{array}$ & $\begin{array}{l}4 \\
4\end{array}$ & $\begin{array}{l}83 \\
84\end{array}$ & $\begin{array}{l}1,370 \\
1,360\end{array}$ \\
\hline $\begin{array}{l}21 \\
20 \\
25 \\
20 \\
30 \\
22 \\
20 \\
40\end{array}$ & $\begin{array}{l}4 \\
4 \\
4 \\
4 \\
4 \\
4 \\
4 \\
8\end{array}$ & $\begin{array}{l}83 \\
34 \\
79 \\
74 \\
79 \\
77 \\
77 \\
68\end{array}$ & $\begin{array}{l}1,370 \\
2,140 \\
1,400 \\
1,450 \\
1,400 \\
1,420 \\
1,420 \\
1,510\end{array}$ \\
\hline $\begin{array}{l}42 \\
30 \\
40 \\
30 \\
25 \\
36 \\
35 \\
25\end{array}$ & $\begin{array}{r}8 \\
8 \\
8 \\
8 \\
8 \\
8 \\
8 \\
15\end{array}$ & $\begin{array}{r}66 \\
78 \\
68 \\
78 \\
83 \\
72 \\
73 \\
290\end{array}$ & $\begin{array}{l}1,530 \\
1,410 \\
1,510 \\
1,410 \\
1,370 \\
1,470 \\
1,460 \\
1,540\end{array}$ \\
\hline $\begin{array}{l}19 \\
21 \\
22\end{array}$ & $\begin{array}{l}10 \\
16 \\
16\end{array}$ & $\begin{array}{l}112 \\
128 \\
127\end{array}$ & $\begin{array}{l}1,670 \\
2,130 \\
2,140\end{array}$ \\
\hline
\end{tabular}

HUDSON WATER WORKS INC. HUDSON WATER WORKS INC. HUDSON WATER WORKS INC. HUDSON WATER WORKS INC. HUDSON WATER WORKS INC. HUDSON WATER WORKS INC. HUDSON WATER WORKS INC. HUDSON WATER WORKS INC.

HUDSON WATER WORKS INC. HUDSON WATER WORKS INC. HUDSON WATER WORKS INC. HUDSON WATER WORKS INC. HUDSON WATER WORKS INC. HUDSON WATER WORKS INC. HUDSON WATER WORKS INC NEW PORT RICHEY, CITY OF

PASCO COUNTY PASCO COUNTY PASCO COUNTY 
Table 5.-Computation of well protection zones for well fields

Sources and explanation of data for calculations:

Well name - From water companies, West Coast Regional Water Supply Authority, and Southwest Florida Water Management District (SWFWMD) files.

Cons. use permit - Consumptive use permit number. From SWFWM permitting files, February 2, 1987.

Lat, Long. Sec. Twn, and Rng - Latitude, longitude, section, township, and range. From SWFimD permitting files, Fobruary 2,1987 .

Av.Q - Permitted average pumping rate, in gallons per day, for public-supply use. From SWFWM permitting files, February 2, 1987 .

Well depth, in feet - From SWFWM and U.S. Geological Survey files (Eldridge-Wilde), and Hutchinson (1985)Cross Bar Ranch.

F1 - Altitude of top of Upper Floridan aquifer, in feet above sea level. From Buono and Rutledge (1978) and Stewart (1968)-Eldridge-Wilde, East Lake Road, south Pasco, Section 21, and Cosme-Odessa.

Lsd - Altitude of land surface, in foot above sea lovel, from USGS 7 -minute topographic maps at latitude and longitude given.

h - Thickness of Upper Floridan aquifer penetrated, in feet. $h=$ Well depth - (Lsd-F1).

$\underline{\underline{r}}-$ Radius of protection zone of the well, rounded to the nearest 10 feet. $r=\left[\frac{1825(\mathrm{Av} .0)}{7.48 \pi \mathrm{hn}}\right] \mathrm{n}=0.05 \mathrm{for}$ the Upper Floridan aquifer.

Area - Area of protection zone of the well, rounded to the nearest 10,000 square feet. Area $=\frac{1825(\mathrm{Av} .0)}{7.48 \mathrm{hn}}$.

[Radius and area not computed where vertical travel time exceeds 5 years]

\begin{tabular}{|c|c|c|c|c|c|c|c|c|c|c|c|c|c|}
\hline $\begin{array}{l}\text { Well } \\
\text { name }\end{array}$ & $\begin{array}{c}\text { Cons } \\
\text { use } \\
\text { permit }\end{array}$ & Lat. & Long. & Sec. & $\begin{array}{l}\text { Twn. } \\
\text { (S.) }\end{array}$ & $\begin{array}{l}\text { Rng; } \\
\text { (E.) }\end{array}$ & $\begin{array}{c}\mathrm{Av} \cdot \mathrm{Q} \\
(\mathrm{gai} / \mathrm{d})\end{array}$ & $\begin{array}{l}\text { Well } \\
\text { depth } \\
\text { (ft) }\end{array}$ & $\begin{array}{l}\text { FI } \\
(\text { sea } \\
\text { lov.) }\end{array}$ & $\begin{array}{l}1 s d \\
(s \bullet a \\
\text { lov.) }\end{array}$ & $\begin{array}{l}h \\
\text { (ft) }\end{array}$ & $\underset{(f t)}{r}$ & $\begin{array}{l}\text { Area } \\
\left(\mathrm{ft}^{2}\right)\end{array}$ \\
\hline \multicolumn{14}{|c|}{ South Pasco } \\
\hline $\begin{array}{l}41 \\
43 \\
44 \\
46 \\
47 \\
48 \\
49 \\
50\end{array}$ & $\begin{array}{l}0364701 \\
0364701 \\
0364701 \\
0364701 \\
0364701 \\
0364701 \\
0364701 \\
0364701\end{array}$ & $\begin{array}{l}281024 \\
281041 \\
281045 \\
281050 \\
281105 \\
281106 \\
281125 \\
281126\end{array}$ & $\begin{array}{l}823059 \\
823046 \\
823028 \\
823101 \\
823108 \\
823121 \\
823058 \\
823038\end{array}$ & $\begin{array}{l}33 \\
33 \\
33 \\
33 \\
33 \\
32 \\
28 \\
28\end{array}$ & $\begin{array}{l}26 \\
26 \\
26 \\
26 \\
26 \\
26 \\
26 \\
26\end{array}$ & $\begin{array}{l}18 \\
18 \\
18 \\
18 \\
18 \\
18 \\
18 \\
18\end{array}$ & $\begin{array}{l}3,000,000 \\
3,000,000 \\
3,000,000 \\
3,000,000 \\
3,000,000 \\
3,000,000 \\
3,000,000 \\
3,000,000\end{array}$ & $\begin{array}{l}707 \\
704 \\
708 \\
653 \\
704 \\
506 \\
706 \\
612\end{array}$ & $\begin{array}{l}5 \\
5 \\
5 \\
5 \\
5 \\
5 \\
5 \\
5\end{array}$ & $\begin{array}{l}59 \\
60 \\
61 \\
58 \\
59 \\
61 \\
60 \\
59\end{array}$ & $\begin{array}{l}653 \\
649 \\
652 \\
600 \\
650 \\
450 \\
651 \\
558\end{array}$ & $\begin{array}{l}2,670 \\
2,680 \\
2,670 \\
2,790 \\
2,680 \\
3,220 \\
2,680 \\
2,890\end{array}$ & $\begin{array}{l}22,420,000 \\
22,560,000 \\
22,450,000 \\
24,400,000 \\
22,520,000 \\
32,530,000 \\
22,490,000 \\
26,230,000\end{array}$ \\
\hline \multicolumn{14}{|c|}{ Cypress Croek } \\
\hline $\begin{array}{l}c-1 \\
C-2 \\
C-3 \\
C-4 \\
C-5 \\
c-6 \\
c-7 \\
c-8\end{array}$ & $\begin{array}{l}0365002 \\
0365002 \\
0365002 \\
0365002 \\
0365002 \\
0365002 \\
0365002 \\
0365002\end{array}$ & $\begin{array}{l}281828 \\
281809 \\
281800 \\
281752 \\
281742 \\
281741 \\
281728 \\
281713\end{array}$ & $\begin{array}{l}822236 \\
822245 \\
822250 \\
822258 \\
822307 \\
822329 \\
822338 \\
822340\end{array}$ & $\begin{array}{l}14 \\
14 \\
23 \\
23 \\
23 \\
22 \\
22 \\
27\end{array}$ & $\begin{array}{l}25 \\
25 \\
25 \\
25 \\
25 \\
25 \\
25 \\
25\end{array}$ & $\begin{array}{l}19 \\
19 \\
19 \\
19 \\
19 \\
19 \\
19 \\
19\end{array}$ & $\begin{array}{l}3,000,000 \\
3,000,000 \\
3,000,000 \\
3,000,000 \\
3,000,000 \\
3,000,000 \\
3,000,000 \\
3,000,000\end{array}$ & $\begin{array}{l}700 \\
700 \\
700 \\
700 \\
700 \\
700 \\
700 \\
700\end{array}$ & $\begin{array}{l}40 \\
40 \\
40 \\
40 \\
40 \\
40 \\
40 \\
40\end{array}$ & $\begin{array}{l}78 \\
79 \\
72 \\
72 \\
72 \\
71 \\
67 \\
66\end{array}$ & $\begin{array}{l}662 \\
661 \\
668 \\
668 \\
668 \\
669 \\
673 \\
674\end{array}$ & $\begin{array}{l}2,650 \\
2,660 \\
2,640 \\
2,640 \\
2,640 \\
2,640 \\
2,630 \\
2,630\end{array}$ & $\begin{array}{l}22,110,000 \\
22,150,000 \\
21,910,000 \\
21,910,000 \\
21,910,000 \\
21,880,000 \\
21,750,000 \\
21,720,000\end{array}$ \\
\hline $\begin{array}{l}c-9 \\
c-10 \\
c-11 \\
c-12 \\
c-13\end{array}$ & $\begin{array}{l}0365002 \\
0365002 \\
0365002 \\
0365002 \\
0365002\end{array}$ & $\begin{array}{l}281650 \\
281641 \\
281804 \\
281743 \\
281738\end{array}$ & $\begin{array}{l}822340 \\
822404 \\
822235 \\
822220 \\
822208\end{array}$ & $\begin{array}{l}27 \\
27 \\
23 \\
23 \\
24\end{array}$ & $\begin{array}{l}25 \\
25 \\
25 \\
25 \\
25\end{array}$ & $\begin{array}{l}19 \\
19 \\
19 \\
19 \\
19\end{array}$ & $\begin{array}{l}3,000,000 \\
3,000,000 \\
3,000,000 \\
3,000,000 \\
3,000,000\end{array}$ & $\begin{array}{l}700 \\
700 \\
700 \\
700 \\
700\end{array}$ & $\begin{array}{l}40 \\
40 \\
40 \\
40 \\
40\end{array}$ & $\begin{array}{l}65 \\
64 \\
72 \\
71 \\
71\end{array}$ & $\begin{array}{l}675 \\
676 \\
668 \\
669 \\
669\end{array}$ & $\begin{array}{l}2,630 \\
2,630 \\
2,640 \\
2,640 \\
2,640\end{array}$ & $\begin{array}{l}21,690,000 \\
21,660,000 \\
21,910,000 \\
21,880,000 \\
21,880,000\end{array}$ \\
\hline \multicolumn{14}{|c|}{ Cross Bar Ranch } \\
\hline $\begin{array}{l}\mathrm{CB}-1 \\
\mathrm{CB}-2 \\
\mathrm{CB}-3 \\
\mathrm{CB}-4 \\
\mathrm{CB}-5 \\
\mathrm{CB}-6 \\
\mathrm{CB}-7 \\
\mathrm{CB}-8\end{array}$ & $\begin{array}{l}0429001 \\
0429001 \\
0429001 \\
0429001 \\
0429001 \\
0429001 \\
0429001 \\
0429001\end{array}$ & $\begin{array}{l}282110 \\
282135 \\
282149 \\
282154 \\
282220 \\
282235 \\
282243 \\
282309\end{array}$ & $\begin{array}{l}822736 \\
822757 \\
822837 \\
822806 \\
822806 \\
822843 \\
822813 \\
822809\end{array}$ & $\begin{array}{l}36 \\
36 \\
26 \\
25 \\
25 \\
23 \\
23 \\
24\end{array}$ & $\begin{array}{l}24 \\
24 \\
24 \\
24 \\
24 \\
24 \\
24 \\
24\end{array}$ & $\begin{array}{l}18 \\
18 \\
18 \\
18 \\
18 \\
18 \\
18 \\
18\end{array}$ & $\begin{array}{l}3,000,000 \\
3,000,000 \\
3,000,000 \\
3,000,000 \\
3,000,000 \\
3,000,000 \\
3,000,000 \\
3,000,000\end{array}$ & $\begin{array}{l}710 \\
702 \\
700 \\
705 \\
705 \\
705 \\
485 \\
710\end{array}$ & $\begin{array}{l}20 \\
20 \\
20 \\
20 \\
30 \\
30 \\
30 \\
30\end{array}$ & $\begin{array}{l}76 \\
78 \\
75 \\
76 \\
71 \\
71 \\
73 \\
71\end{array}$ & $\begin{array}{l}654 \\
644 \\
645 \\
649 \\
664 \\
664 \\
442 \\
669\end{array}$ & $\begin{array}{l}2,670 \\
2,690 \\
2,690 \\
2,680 \\
2,650 \\
2,650 \\
3,250 \\
2,640\end{array}$ & $\begin{array}{l}22,380,000 \\
22,730,000 \\
22,700,000 \\
22,560,000 \\
22,050,000 \\
22,050,000 \\
33,120,000 \\
21,880,000\end{array}$ \\
\hline $\begin{array}{r}C B-9 \\
C B-10 \\
C B-11 \\
C B-12 \\
C B-13 \\
C B-14 \\
C B-15 \\
C B-16 A\end{array}$ & $\begin{array}{l}0429001 \\
0429001 \\
0429001 \\
0429001 \\
0429001 \\
0429001 \\
0429001 \\
0429001\end{array}$ & $\begin{array}{l}282326 \\
282340 \\
282347 \\
282347 \\
282413 \\
282412 \\
282412 \\
282439\end{array}$ & $\begin{array}{l}822821 \\
822752 \\
822715 \\
822645 \\
822714 \\
822643 \\
822751 \\
822743\end{array}$ & $\begin{array}{l}14 \\
13 \\
13 \\
18 \\
13 \\
18 \\
13 \\
12\end{array}$ & $\begin{array}{l}24 \\
24 \\
24 \\
24 \\
24 \\
24 \\
24 \\
24\end{array}$ & $\begin{array}{l}18 \\
18 \\
18 \\
19 \\
18 \\
19 \\
18 \\
18\end{array}$ & $\begin{array}{l}3,000,000 \\
3,000,000 \\
3,000,000 \\
3,000,000 \\
3,000,000 \\
3,000,000 \\
3,000,000 \\
3,000,000\end{array}$ & $\begin{array}{l}703 \\
710 \\
702 \\
710 \\
700 \\
710 \\
710 \\
630\end{array}$ & $\begin{array}{l}30 \\
30 \\
30 \\
30 \\
30 \\
30 \\
30 \\
30\end{array}$ & $\begin{array}{l}70 \\
70 \\
72 \\
72 \\
72 \\
72 \\
68 \\
60\end{array}$ & $\begin{array}{l}663 \\
670 \\
660 \\
668 \\
658 \\
668 \\
672 \\
600\end{array}$ & $\begin{array}{l}2,650 \\
2,640 \\
2,660 \\
2,640 \\
2,660 \\
2,640 \\
2,630 \\
2,790\end{array}$ & $\begin{array}{l}22,080,000 \\
21,850,000 \\
22,180,000 \\
21,910,000 \\
22,250,000 \\
21,910,000 \\
21,780,000 \\
24,400,000\end{array}$ \\
\hline$C B-17$ & 0429001 & 282445 & 822639 & 7 & 24 & 19 & $3,000,000$ & 710 & 30 & 75 & 665 & 2,650 & $22,010,000$ \\
\hline
\end{tabular}


Table 5. - Computation of well protection zones for well fields - Continued

\begin{tabular}{|c|c|c|c|c|c|c|c|c|c|c|c|c|c|}
\hline $\begin{array}{l}\text { Well } \\
\text { name }\end{array}$ & $\begin{array}{c}\text { Cons. } \\
\text { use } \\
\text { permit }\end{array}$ & Lat. & Long. & Sec. & Tw. & $\begin{array}{l}\text { Rng; } \\
\text { (E.; }\end{array}$ & $\begin{array}{c}\text { Av. } Q \\
(\mathrm{gai} / \mathrm{d})\end{array}$ & $\begin{array}{l}\text { Well } \\
\text { depth } \\
\text { (ft) }\end{array}$ & $\begin{array}{l}\text { FI } \\
(s e a \\
\text { lev.) }\end{array}$ & $\begin{array}{l}\text { Isd } \\
(s e a \\
\text { Lev.) }\end{array}$ & $\begin{array}{c}h \\
\text { (ft) }\end{array}$ & $\stackrel{r}{f t}$ & $\begin{array}{l}\text { Arga } \\
\left(\mathrm{ft}^{2}\right)\end{array}$ \\
\hline \multicolumn{14}{|c|}{ Starkey } \\
\hline $\begin{array}{l}W-1 \\
W-2 \\
W-3 \\
W-4 \\
W-6\end{array}$ & $\begin{array}{l}0444602 \\
0444602 \\
0444602 \\
0444602 \\
0444602\end{array}$ & $\begin{array}{l}281530 \\
281513 \\
281500 \\
281526 \\
281500\end{array}$ & $\begin{array}{l}823839 \\
823849 \\
823847 \\
823812 \\
823624\end{array}$ & $\begin{array}{l}6 \\
6 \\
6 \\
6 \\
4\end{array}$ & $\begin{array}{l}26 \\
26 \\
26 \\
26 \\
26\end{array}$ & $\begin{array}{l}17 \\
17 \\
17 \\
17 \\
17\end{array}$ & $\begin{array}{l}350,000 \\
100,000 \\
350,000 \\
200,000 \\
740,000\end{array}$ & $\begin{array}{l}300 \\
550 \\
345 \\
300 \\
750\end{array}$ & $\begin{array}{r}17 \\
18 \\
20 \\
20 \\
0\end{array}$ & $\begin{array}{l}22 \\
22 \\
36 \\
31 \\
28\end{array}$ & $\begin{array}{l}268 \\
518 \\
299 \\
259 \\
712\end{array}$ & $\begin{array}{r}1,420 \\
550 \\
1,350 \\
1,100 \\
1,270\end{array}$ & $\begin{array}{r}5,790,000 \\
890,000 \\
5,190,000 \\
3,380,000 \\
5,000,000\end{array}$ \\
\hline $\begin{array}{l}W-7 \\
W-8 \\
W-9 \\
W-10 \\
W-11 \\
W-12 \\
W-13 \\
W-14 \\
W-15\end{array}$ & $\begin{array}{l}0444602 \\
0444602 \\
0444602 \\
0444602 \\
0444602 \\
0444602 \\
0444602 \\
0444602 \\
0444602\end{array}$ & $\begin{array}{l}281449 \\
281500 \\
281449 \\
281500 \\
281437 \\
281500 \\
281437 \\
281500 \\
281450\end{array}$ & $\begin{array}{l}823622 \\
823541 \\
823542 \\
823511 \\
823511 \\
823426 \\
823427 \\
823343 \\
823358\end{array}$ & $\begin{array}{r}4 \\
3 \\
3 \\
3 \\
10 \\
2 \\
11 \\
1 \\
1\end{array}$ & $\begin{array}{l}26 \\
26 \\
26 \\
26 \\
26 \\
26 \\
26 \\
26 \\
26\end{array}$ & $\begin{array}{l}17 \\
17 \\
17 \\
17 \\
17 \\
17 \\
17 \\
17 \\
17\end{array}$ & $\begin{array}{l}740,000 \\
740,000 \\
740,000 \\
740,000 \\
740,000 \\
740,000 \\
740,000 \\
740,000 \\
740,000\end{array}$ & $\begin{array}{l}750 \\
750 \\
750 \\
750 \\
750 \\
750 \\
750 \\
750 \\
750\end{array}$ & $\begin{array}{l}0 \\
0 \\
0 \\
0 \\
0 \\
0 \\
0 \\
0 \\
0\end{array}$ & $\begin{array}{l}39 \\
41 \\
47 \\
44 \\
43 \\
43 \\
45 \\
48 \\
48\end{array}$ & $\begin{array}{l}711 \\
709 \\
703 \\
706 \\
707 \\
707 \\
705 \\
702 \\
702\end{array}$ & $\begin{array}{l}1,270 \\
1,270 \\
1,280 \\
1,280 \\
1,280 \\
1,280 \\
1,280 \\
1,280 \\
1,280\end{array}$ & $\begin{array}{r}5,080,000 \\
5,090,000 \\
5,140,000 \\
5,110,000 \\
5,110,000 \\
5,110,000 \\
5,120,000 \\
5,140,000 \\
5,140,000\end{array}$ \\
\hline \multicolumn{14}{|c|}{ Eldridge-Wilde } \\
\hline $\begin{array}{r}5 B \\
5 S \\
5 N \\
6 S \\
7 S \\
6 N \\
8 S \\
101\end{array}$ & $\begin{array}{l}0267301 \\
0267301 \\
0267301 \\
0267301 \\
0267301 \\
0267301 \\
0267301 \\
0267301\end{array}$ & $\begin{array}{l}280902 \\
280852 \\
280922 \\
280844 \\
280856 \\
280922 \\
280851 \\
281021\end{array}$ & $\begin{array}{l}823945 \\
823942 \\
823950 \\
823931 \\
823935 \\
823944 \\
823924 \\
823934\end{array}$ & $\begin{array}{r}12 \\
12 \\
12 \\
12 \\
12 \\
12 \\
12 \\
1\end{array}$ & $\begin{array}{l}27 \\
27 \\
27 \\
27 \\
27 \\
27 \\
27 \\
27\end{array}$ & $\begin{array}{l}16 \\
16 \\
16 \\
16 \\
16 \\
16 \\
16 \\
16\end{array}$ & $\begin{array}{l}948,276 \\
948,276 \\
948,276 \\
948,276 \\
948,276 \\
948,276 \\
948,276 \\
948,276\end{array}$ & $\begin{array}{l}300 \\
175 \\
340 \\
140 \\
290 \\
346 \\
245 \\
311\end{array}$ & $\begin{array}{l}-25 \\
-25 \\
-25 \\
-25 \\
-25 \\
-25 \\
-25 \\
-15\end{array}$ & $\begin{array}{l}28 \\
26 \\
30 \\
28 \\
28 \\
31 \\
31 \\
30\end{array}$ & $\begin{array}{r}247 \\
124 \\
285 \\
87 \\
237 \\
290 \\
189 \\
266\end{array}$ & $\begin{array}{l}2,440 \\
3,450 \\
2,270 \\
4,110 \\
2,490 \\
2,250 \\
2,790 \\
2,350\end{array}$ & $\begin{array}{l}18,730,000 \\
37,320,000 \\
16,240,000 \\
53,190,000 \\
19,520,000 \\
15,960,000 \\
24,480,000 \\
17,400,000\end{array}$ \\
\hline $\begin{array}{l}102 \\
103 \\
104 \\
105 \\
106 \\
107 \\
109 \\
110\end{array}$ & $\begin{array}{l}0267301 \\
0267301 \\
0267301 \\
0267301 \\
0267301 \\
0267301 \\
0267301 \\
0267301\end{array}$ & $\begin{array}{l}281013 \\
281021 \\
281011 \\
281017 \\
281021 \\
281013 \\
281000 \\
280952\end{array}$ & $\begin{array}{l}823935 \\
823926 \\
823925 \\
823919 \\
823907 \\
823907 \\
823916 \\
823916\end{array}$ & $\begin{array}{l}1 \\
1 \\
1 \\
1 \\
1 \\
1 \\
1 \\
1\end{array}$ & $\begin{array}{l}27 \\
27 \\
27 \\
27 \\
27 \\
27 \\
27 \\
27\end{array}$ & $\begin{array}{l}16 \\
16 \\
16 \\
16 \\
16 \\
16 \\
16 \\
16\end{array}$ & $\begin{array}{l}948,276 \\
948,276 \\
948,276 \\
948,276 \\
948,276 \\
948,276 \\
948,276 \\
948,276\end{array}$ & $\begin{array}{l}316 \\
310 \\
402 \\
250 \\
345 \\
308 \\
384 \\
333\end{array}$ & $\begin{array}{l}-20 \\
-15 \\
-15 \\
-10 \\
-10 \\
-10 \\
-15 \\
-20\end{array}$ & $\begin{array}{l}29 \\
31 \\
32 \\
33 \\
34 \\
37 \\
33 \\
31\end{array}$ & $\begin{array}{l}267 \\
264 \\
355 \\
207 \\
301 \\
261 \\
336 \\
282\end{array}$ & $\begin{array}{l}2,350 \\
2,360 \\
2,040 \\
2,670 \\
2,210 \\
2,380 \\
2,090 \\
2,290\end{array}$ & $\begin{array}{l}17,330,000 \\
17,530,000 \\
13,030,000 \\
22,350,000 \\
15,370,000 \\
17,730,000 \\
13,770,000 \\
16,410,000\end{array}$ \\
\hline $\begin{array}{l}111 \\
112 \\
113 \\
114 \\
115 \\
116 \\
117 \\
118\end{array}$ & $\begin{array}{l}0267301 \\
0267301 \\
0267301 \\
0267301 \\
0267301 \\
0267301 \\
0267301 \\
0267301\end{array}$ & $\begin{array}{l}281008 \\
280958 \\
280942 \\
280932 \\
280944 \\
280938 \\
280927 \\
280927\end{array}$ & $\begin{array}{l}823916 \\
823905 \\
823906 \\
823916 \\
823927 \\
823923 \\
823948 \\
823927\end{array}$ & $\begin{array}{r}1 \\
1 \\
1 \\
1 \\
1 \\
1 \\
12 \\
12\end{array}$ & $\begin{array}{l}27 \\
27 \\
27 \\
27 \\
27 \\
27 \\
27 \\
27\end{array}$ & $\begin{array}{l}16 \\
16 \\
16 \\
16 \\
16 \\
16 \\
16 \\
16\end{array}$ & $\begin{array}{l}948,276 \\
948,276 \\
948,276 \\
948,276 \\
948,276 \\
948,276 \\
948,276 \\
948,276\end{array}$ & $\begin{array}{l}399 \\
314 \\
503 \\
407 \\
321 \\
400 \\
302 \\
407\end{array}$ & $\begin{array}{l}-15 \\
-15 \\
-20 \\
-20 \\
-20 \\
-20 \\
-20 \\
-20\end{array}$ & $\begin{array}{l}33 \\
35 \\
35 \\
30 \\
32 \\
34 \\
29 \\
31\end{array}$ & $\begin{array}{l}351 \\
264 \\
448 \\
357 \\
269 \\
346 \\
253 \\
356\end{array}$ & $\begin{array}{l}2,050 \\
2,360 \\
1,810 \\
2,030 \\
2,340 \\
2,060 \\
2,410 \\
2,030\end{array}$ & $\begin{array}{l}13,180,000 \\
17,530,000 \\
10,330,000 \\
12,960,000 \\
17,200,000 \\
13,370,000 \\
18,290,000 \\
13,000,000\end{array}$ \\
\hline $\begin{array}{l}119 \\
120 \\
121 \\
122 \\
131 \\
134 \\
135 \\
136\end{array}$ & $\begin{array}{l}0267301 \\
0267301 \\
0267301 \\
0267301 \\
0267301 \\
0267301 \\
0267301 \\
0267301\end{array}$ & $\begin{array}{l}280923 \\
280915 \\
280926 \\
280931 \\
281020 \\
281020 \\
281013 \\
281020\end{array}$ & $\begin{array}{l}823927 \\
823904 \\
823906 \\
823904 \\
823858 \\
823844 \\
823846 \\
823831\end{array}$ & $\begin{array}{r}12 \\
12 \\
12 \\
1 \\
6 \\
6 \\
6 \\
6\end{array}$ & $\begin{array}{l}27 \\
27 \\
27 \\
27 \\
27 \\
27 \\
27 \\
27\end{array}$ & $\begin{array}{l}16 \\
16 \\
16 \\
16 \\
17 \\
17 \\
17 \\
17\end{array}$ & $\begin{array}{l}948,276 \\
948,276 \\
948,276 \\
948,276 \\
948,276 \\
948,276 \\
948,276 \\
948,276\end{array}$ & $\begin{array}{l}107 \\
809 \\
770 \\
291 \\
460 \\
275 \\
286 \\
440\end{array}$ & $\begin{array}{l}-20 \\
-20 \\
-20 \\
-20 \\
-10 \\
-10 \\
-10 \\
-10\end{array}$ & $\begin{array}{l}30 \\
26 \\
10 \\
37 \\
37 \\
37 \\
38 \\
36\end{array}$ & $\begin{array}{l}357 \\
763 \\
710 \\
234 \\
413 \\
228 \\
238 \\
394\end{array}$ & $\begin{array}{l}2,030 \\
1,390 \\
1,440 \\
2,510 \\
1,890 \\
2,540 \\
2,490 \\
1,930\end{array}$ & $\begin{array}{r}12,960,000 \\
6,060,000 \\
6,520,000 \\
19,770,000 \\
11,200,000 \\
20,300,000 \\
19,440,000 \\
11,740,000\end{array}$ \\
\hline $\begin{array}{r}137 \\
138 \\
139 \\
140 \\
141 \\
142 \\
1 \\
2\end{array}$ & $\begin{array}{l}0267301 \\
0267301 \\
0267301 \\
0267301 \\
0267301 \\
0267301 \\
0267301 \\
0267301\end{array}$ & $\begin{array}{l}281013 \\
280958 \\
280916 \\
281020 \\
281009 \\
281014 \\
280906 \\
280904\end{array}$ & $\begin{array}{l}823826 \\
823811 \\
823812 \\
823815 \\
823810 \\
823809 \\
824028 \\
824023\end{array}$ & $\begin{array}{r}6 \\
6 \\
6 \\
6 \\
6 \\
6 \\
11 \\
11\end{array}$ & $\begin{array}{l}27 \\
27 \\
27 \\
27 \\
27 \\
27 \\
27 \\
27\end{array}$ & $\begin{array}{l}17 \\
17 \\
17 \\
17 \\
17 \\
17 \\
16 \\
16\end{array}$ & $\begin{array}{l}948,276 \\
948,276 \\
948,276 \\
948,276 \\
948,276 \\
948,276 \\
948,276 \\
948,276\end{array}$ & $\begin{array}{l}330 \\
400 \\
560 \\
440 \\
510 \\
400 \\
300 \\
297\end{array}$ & $\begin{array}{l}-10 \\
-10 \\
-10 \\
-10 \\
-10 \\
-10 \\
-30 \\
-30\end{array}$ & $\begin{array}{l}37 \\
40 \\
42 \\
37 \\
39 \\
38 \\
24 \\
24\end{array}$ & $\begin{array}{l}283 \\
350 \\
508 \\
393 \\
461 \\
352 \\
246 \\
243\end{array}$ & $\begin{array}{l}2,280 \\
2,050 \\
1,700 \\
1,940 \\
1,790 \\
2,050 \\
2,450 \\
2,460\end{array}$ & $\begin{array}{r}16,350,000 \\
13,220,000 \\
9,110,000 \\
11,770,000 \\
10,040,000 \\
13,150,000 \\
18,810,000 \\
19,040,000\end{array}$ \\
\hline $\begin{array}{r}4 \\
5 \\
6 \\
7 \\
8 \\
9 \\
10 \AA \\
11 \AA\end{array}$ & $\begin{array}{l}0267301 \\
0267301 \\
0267301 \\
0267301 \\
0267301 \\
0267301 \\
0267301 \\
0267301\end{array}$ & $\begin{array}{l}280904 \\
280903 \\
280903 \\
280908 \\
280903 \\
280911 \\
280918 \\
280902\end{array}$ & $\begin{array}{l}823958 \\
823953 \\
823933 \\
823925 \\
823920 \\
823912 \\
823912 \\
823912\end{array}$ & $\begin{array}{l}12 \\
12 \\
12 \\
12 \\
12 \\
12 \\
12 \\
12\end{array}$ & $\begin{array}{l}27 \\
27 \\
27 \\
27 \\
27 \\
27 \\
27 \\
27\end{array}$ & $\begin{array}{l}16 \\
16 \\
16 \\
16 \\
16 \\
16 \\
16 \\
16\end{array}$ & $\begin{array}{l}948,276 \\
948,276 \\
948,276 \\
948,276 \\
948,276 \\
948,276 \\
948,276 \\
948,276\end{array}$ & $\begin{array}{l}300 \\
300 \\
300 \\
285 \\
300 \\
302 \\
550 \\
300\end{array}$ & $\begin{array}{l}-30 \\
-25 \\
-25 \\
-25 \\
-25 \\
-20 \\
-20 \\
-20\end{array}$ & $\begin{array}{l}28 \\
28 \\
30 \\
34 \\
34 \\
32 \\
32 \\
29\end{array}$ & $\begin{array}{l}242 \\
247 \\
245 \\
226 \\
241 \\
250 \\
498 \\
251\end{array}$ & $\begin{array}{l}2,470 \\
2,440 \\
2,450 \\
2,550 \\
2,470 \\
2,430 \\
1,720 \\
2,420\end{array}$ & $\begin{array}{r}19,120,000 \\
18,730,000 \\
18,890,000 \\
20,470,000 \\
19,200,000 \\
18,510,000 \\
9,290,000 \\
18,440,000\end{array}$ \\
\hline
\end{tabular}


Table 5. - Computation of well protection zones for well fields - Continued

\begin{tabular}{|c|c|c|c|c|c|c|c|c|c|c|c|c|c|}
\hline $\begin{array}{l}\text { Well } \\
\text { name }\end{array}$ & $\begin{array}{c}\text { Cons. } \\
\text { use } \\
\text { permit }\end{array}$ & Lat. & Long. & Sec. & $\begin{array}{l}\text { Twn: } \\
(S .)\end{array}$ & $\begin{array}{l}\text { Rng; ; } \\
\text { (E,) }\end{array}$ & $\begin{array}{l}\text { Av.Q } \\
\text { (gai/d) }\end{array}$ & $\begin{array}{l}\text { Well } \\
\text { depth } \\
\text { (ft) }\end{array}$ & $\begin{array}{l}\text { F1 } \\
\text { (sea } \\
\text { lev. })\end{array}$ & $\begin{array}{l}18 d \\
(s e a \\
1 e v,)\end{array}$ & $\begin{array}{c}h \\
(f t)\end{array}$ & $\stackrel{r}{(f t)}$ & $\begin{array}{r}\text { Arga } \\
\left.(\mathrm{ft})^{2}\right)\end{array}$ \\
\hline \multicolumn{14}{|c|}{ Eldridge-Wilde--Continued } \\
\hline $\begin{array}{l}12 \\
13 \\
1 S \\
2 A \\
3 S \\
3 B \\
4 \mathrm{~N} \\
4 \mathrm{~S}\end{array}$ & $\begin{array}{l}0267301 \\
0267301 \\
0267301 \\
0267301 \\
0267301 \\
0267301 \\
0267301 \\
0267301\end{array}$ & $\begin{array}{l}280853 \\
280845 \\
280852 \\
280856 \\
280900 \\
280902 \\
280917 \\
280851\end{array}$ & $\begin{array}{l}823904 \\
823905 \\
824005 \\
824015 \\
824024 \\
824006 \\
823958 \\
823955\end{array}$ & $\begin{array}{l}12 \\
12 \\
11 \\
11 \\
11 \\
11 \\
12 \\
12\end{array}$ & $\begin{array}{l}27 \\
27 \\
27 \\
27 \\
27 \\
27 \\
27 \\
27\end{array}$ & $\begin{array}{l}16 \\
16 \\
16 \\
16 \\
16 \\
16 \\
16 \\
16\end{array}$ & $\begin{array}{l}948,276 \\
948,276 \\
948,276 \\
948,276 \\
948,276 \\
948,276 \\
948,276 \\
948,276\end{array}$ & $\begin{array}{l}300 \\
320 \\
300 \\
450 \\
309 \\
410 \\
300 \\
285\end{array}$ & $\begin{array}{l}-20 \\
-25 \\
-30 \\
-30 \\
-30 \\
-30 \\
-25 \\
-30\end{array}$ & $\begin{array}{l}29 \\
29 \\
29 \\
25 \\
23 \\
26 \\
27 \\
26\end{array}$ & $\begin{array}{l}251 \\
266 \\
241 \\
395 \\
256 \\
354 \\
248 \\
229\end{array}$ & $\begin{array}{l}2,420 \\
2,350 \\
2,470 \\
1,930 \\
2,400 \\
2,040 \\
2,440 \\
2,540\end{array}$ & $\begin{array}{l}18,440,000 \\
17,400,000 \\
19,200,000 \\
11,710,000 \\
18,080,000 \\
13,070,000 \\
18,660,000 \\
20,210,000\end{array}$ \\
\hline $\begin{array}{l}4 A \\
5 A\end{array}$ & $\begin{array}{l}0267301 \\
0267301\end{array}$ & $\begin{array}{l}280902 \\
280917\end{array}$ & $\begin{array}{l}823957 \\
823946\end{array}$ & $\begin{array}{l}12 \\
12\end{array}$ & $\begin{array}{l}27 \\
27\end{array}$ & $\begin{array}{l}16 \\
16\end{array}$ & $\begin{array}{l}948,276 \\
948,276\end{array}$ & $\begin{array}{l}210 \\
295\end{array}$ & $\begin{array}{l}-25 \\
-25\end{array}$ & $\begin{array}{l}27 \\
30\end{array}$ & $\begin{array}{l}158 \\
240\end{array}$ & $\begin{array}{l}3,050 \\
2,480\end{array}$ & $\begin{array}{l}29,290,000 \\
19,280,000\end{array}$ \\
\hline \multicolumn{14}{|c|}{ East Lake Road } \\
\hline $\begin{array}{l}E L-1 \\
E L-2 \\
E L-3 \\
E L-4 \\
E L-5 \\
E L-6 \\
E L-7 \\
E L-8\end{array}$ & $\begin{array}{l}0439101 \\
0439101 \\
0439101 \\
0439101 \\
0439101 \\
0439101 \\
0439101 \\
0439101\end{array}$ & $\begin{array}{l}280631 \\
280614 \\
280603 \\
280556 \\
280617 \\
280639 \\
280532 \\
280511\end{array}$ & $\begin{array}{l}824117 \\
824117 \\
824109 \\
824056 \\
824056 \\
824055 \\
824057 \\
824056\end{array}$ & $\begin{array}{l}27 \\
27 \\
27 \\
35 \\
26 \\
26 \\
34 \\
34\end{array}$ & $\begin{array}{l}27 \\
27 \\
27 \\
27 \\
27 \\
27 \\
27 \\
27\end{array}$ & $\begin{array}{l}16 \\
16 \\
16 \\
16 \\
16 \\
16 \\
16 \\
16\end{array}$ & $\begin{array}{l}\mathbf{6 2 5}, 000 \\
625,000 \\
625,000 \\
625,000 \\
625,000 \\
625,000 \\
625,000 \\
625,000\end{array}$ & $\begin{array}{l}200 \\
125 \\
169 \\
235 \\
220 \\
200 \\
220 \\
200\end{array}$ & $\begin{array}{l}-25 \\
-25 \\
-25 \\
-25 \\
-25 \\
-25 \\
-25 \\
-25\end{array}$ & $\begin{array}{l}16 \\
17 \\
14 \\
15 \\
14 \\
16 \\
14 \\
14\end{array}$ & $\begin{array}{r}159 \\
83 \\
130 \\
195 \\
181 \\
159 \\
181 \\
161\end{array}$ & & \\
\hline \multicolumn{14}{|c|}{ Section 21} \\
\hline $\begin{array}{l}21-2 \\
2110 \\
21-9 \\
21-8 \\
21-5 \\
21-6 \\
21-1 \\
21-3\end{array}$ & $\begin{array}{l}0000301 \\
0000301 \\
0000301 \\
0000301 \\
0000301 \\
0000301 \\
0000301 \\
0000301\end{array}$ & $\begin{array}{l}280709 \\
280652 \\
280708 \\
280721 \\
280738 \\
280738 \\
280724 \\
280655\end{array}$ & $\begin{array}{l}823059 \\
823011 \\
823011 \\
823011 \\
823034 \\
823020 \\
823057 \\
823058\end{array}$ & $\begin{array}{l}21 \\
21 \\
21 \\
21 \\
21 \\
21 \\
21 \\
21\end{array}$ & $\begin{array}{l}27 \\
27 \\
27 \\
27 \\
27 \\
27 \\
27 \\
27\end{array}$ & $\begin{array}{l}18 \\
18 \\
18 \\
18 \\
18 \\
18 \\
18 \\
18\end{array}$ & $\begin{array}{l}3,000,000 \\
3,000,000 \\
3,000,000 \\
3,000,000 \\
3,000,000 \\
3,000,000 \\
3,000,000 \\
3,000,000\end{array}$ & $\begin{array}{l}412 \\
411 \\
601 \\
551 \\
602 \\
412 \\
571 \\
412\end{array}$ & $\begin{array}{l}10 \\
15 \\
15 \\
15 \\
10 \\
10 \\
10 \\
10\end{array}$ & $\begin{array}{l}55 \\
54 \\
58 \\
56 \\
57 \\
59 \\
55 \\
55\end{array}$ & $\begin{array}{l}367 \\
372 \\
558 \\
510 \\
555 \\
363 \\
526 \\
367\end{array}$ & $\begin{array}{l}3,560 \\
3,540 \\
2,890 \\
3,020 \\
2,900 \\
3,580 \\
2,980 \\
3,560\end{array}$ & $\begin{array}{l}39,890,000 \\
39,350,000 \\
26,230,000 \\
28,700,000 \\
26,380,000 \\
40,330,000 \\
27,830,000 \\
39,890,000\end{array}$ \\
\hline \multicolumn{14}{|c|}{ Cosme-Odesse } \\
\hline $\begin{array}{r}1 \mathrm{C} \\
2 \\
3 \mathrm{~A} \\
4 \\
5 \\
6 \mathrm{~A} \\
7 \mathrm{~A} \\
8\end{array}$ & $\begin{array}{l}0000401 \\
0000401 \\
0000401 \\
0000401 \\
0000401 \\
0000401 \\
0000401 \\
0000401\end{array}$ & $\begin{array}{l}280550 \\
280550 \\
280606 \\
280611 \\
280622 \\
280601 \\
280643 \\
280649\end{array}$ & $\begin{array}{l}823549 \\
823543 \\
823529 \\
823516 \\
823514 \\
823508 \\
823426 \\
823420\end{array}$ & $\begin{array}{l}34 \\
34 \\
27 \\
27 \\
27 \\
27 \\
26 \\
26\end{array}$ & $\begin{array}{l}27 \\
27 \\
27 \\
27 \\
27 \\
27 \\
27 \\
27\end{array}$ & $\begin{array}{l}17 \\
17 \\
17 \\
17 \\
17 \\
17 \\
17 \\
17\end{array}$ & $\begin{array}{r}945,000 \\
565,000 \\
445,000 \\
1,000,000 \\
1,000,000 \\
965,000 \\
945,000 \\
1,000,000\end{array}$ & $\begin{array}{l}345 \\
305 \\
335 \\
345 \\
350 \\
333 \\
324 \\
345\end{array}$ & $\begin{array}{l}-20 \\
-15 \\
-15 \\
-15 \\
-10 \\
-10 \\
-10 \\
-5\end{array}$ & $\begin{array}{l}43 \\
44 \\
45 \\
49 \\
49 \\
47 \\
54 \\
54\end{array}$ & $\begin{array}{l}282 \\
246 \\
275 \\
281 \\
291 \\
276 \\
260 \\
286\end{array}$ & $\begin{array}{l}2,280 \\
1,890 \\
1,590 \\
2,350 \\
2,310 \\
2,330 \\
2,380 \\
2,330\end{array}$ & $\begin{array}{r}16,350,000 \\
11,210,000 \\
7,900,000 \\
17,370,000 \\
16,770,000 \\
17,060,000 \\
17,740,000 \\
17,060,000\end{array}$ \\
\hline $\begin{array}{r}9 A \\
10 \\
12 A \\
16 \\
18 \\
19 \\
20 \\
21\end{array}$ & $\begin{array}{l}0000401 \\
0000401 \\
0000401 \\
0000401 \\
0000401 \\
0000401 \\
0000401 \\
0000401\end{array}$ & $\begin{array}{l}280635 \\
280652 \\
280713 \\
280600 \\
280601 \\
280608 \\
280614 \\
280622\end{array}$ & $\begin{array}{l}823504 \\
823417 \\
823413 \\
823503 \\
823518 \\
823503 \\
823530 \\
823533\end{array}$ & $\begin{array}{l}27 \\
23 \\
23 \\
27 \\
27 \\
27 \\
27 \\
27\end{array}$ & $\begin{array}{l}27 \\
27 \\
27 \\
27 \\
27 \\
27 \\
27 \\
27\end{array}$ & $\begin{array}{l}17 \\
17 \\
17 \\
17 \\
17 \\
17 \\
17 \\
17\end{array}$ & $\begin{array}{r}980,000 \\
995,000 \\
995,000 \\
875,000 \\
545,000 \\
985,000 \\
965,000 \\
1,000,000\end{array}$ & $\begin{array}{l}318 \\
300 \\
300 \\
300 \\
320 \\
312 \\
310 \\
320\end{array}$ & $\begin{array}{r}-10 \\
-5 \\
0 \\
-10 \\
-15 \\
-10 \\
-15 \\
-15\end{array}$ & $\begin{array}{l}52 \\
53 \\
56 \\
50 \\
44 \\
48 \\
48 \\
49\end{array}$ & $\begin{array}{l}256 \\
242 \\
244 \\
240 \\
261 \\
254 \\
247 \\
256\end{array}$ & $\begin{array}{l}2,440 \\
2,530 \\
2,520 \\
2,380 \\
1,800 \\
2,450 \\
2,460 \\
2,460\end{array}$ & $\begin{array}{l}18,680,000 \\
20,060,000 \\
19,900,000 \\
17,790,000 \\
10,190,000 \\
18,920,000 \\
19,060,000 \\
19,060,000\end{array}$ \\
\hline $\begin{array}{l}23 \\
24 \\
25 \\
30 \\
31 \\
32 \\
34\end{array}$ & $\begin{array}{l}0000401 \\
0000401 \\
0000401 \\
0000401 \\
0000401 \\
0000401 \\
0000401\end{array}$ & $\begin{array}{l}280714 \\
280721 \\
280733 \\
280744 \\
280752 \\
280758 \\
280812\end{array}$ & $\begin{array}{l}823420 \\
823421 \\
823421 \\
823429 \\
823430 \\
823433 \\
823440\end{array}$ & $\begin{array}{l}23 \\
23 \\
23 \\
14 \\
14 \\
14 \\
11\end{array}$ & $\begin{array}{l}27 \\
27 \\
27 \\
27 \\
27 \\
27 \\
27\end{array}$ & $\begin{array}{l}17 \\
17 \\
17 \\
17 \\
17 \\
17 \\
17\end{array}$ & $\begin{array}{l}570,000 \\
725,000 \\
785,000 \\
545,000 \\
645,000 \\
785,000 \\
790,000\end{array}$ & $\begin{array}{l}354 \\
357 \\
350 \\
384 \\
374 \\
334 \\
407\end{array}$ & $\begin{array}{l}0 \\
0 \\
0 \\
0 \\
0 \\
0 \\
0\end{array}$ & $\begin{array}{l}50 \\
58 \\
56 \\
61 \\
64 \\
62 \\
62\end{array}$ & $\begin{array}{l}304 \\
299 \\
294 \\
323 \\
310 \\
272 \\
345\end{array}$ & $\begin{array}{l}1,710 \\
1,940 \\
2,040 \\
1,620 \\
1,800 \\
2,120 \\
1,890\end{array}$ & $\begin{array}{r}9,150,000 \\
11,830,000 \\
13,030,000 \\
8,230,000 \\
10,150,000 \\
14,080,000 \\
11,170,000\end{array}$ \\
\hline \multicolumn{14}{|c|}{ Morris Bridge } \\
\hline $\begin{array}{l}150 \\
151 \\
152 \\
153 \\
154 \\
155 \\
156 \\
157\end{array}$ & $\begin{array}{l}0418000 \\
0418000 \\
0418000 \\
0418000 \\
0418000 \\
0418000 \\
0418000 \\
0418000\end{array}$ & $\begin{array}{l}280718 \\
280733 \\
280705 \\
280728 \\
280717 \\
280732 \\
280721 \\
280736\end{array}$ & $\begin{array}{l}822107 \\
822103 \\
822023 \\
822108 \\
822004 \\
821944 \\
821924 \\
821909\end{array}$ & $\begin{array}{l}19 \\
19 \\
19 \\
19 \\
20 \\
20 \\
20 \\
20\end{array}$ & $\begin{array}{l}27 \\
27 \\
27 \\
27 \\
27 \\
27 \\
27 \\
27\end{array}$ & $\begin{array}{l}20 \\
20 \\
20 \\
20 \\
20 \\
20 \\
20 \\
20\end{array}$ & $\begin{array}{l}1,200,000 \\
1,000,000 \\
1,100,000 \\
1,000,000 \\
1,100,000 \\
1,100,000 \\
1,100,000 \\
1,000,000\end{array}$ & $\begin{array}{l}628 \\
610 \\
572 \\
565 \\
550 \\
567 \\
574 \\
614\end{array}$ & $\begin{array}{r}0 \\
0 \\
0 \\
0 \\
0 \\
20 \\
20 \\
20\end{array}$ & $\begin{array}{l}42 \\
46 \\
43 \\
40 \\
42 \\
44 \\
43 \\
45\end{array}$ & $\begin{array}{l}586 \\
564 \\
529 \\
525 \\
508 \\
543 \\
551 \\
589\end{array}$ & $\begin{array}{l}1,780 \\
1,660 \\
1,800 \\
1,720 \\
1,830 \\
1,770 \\
1,760 \\
1,620\end{array}$ & $\begin{array}{r}9,990,000 \\
8,650,000 \\
10,150,000 \\
9,290,000 \\
10,570,000 \\
9,890,000 \\
9,740,000 \\
8,280,000\end{array}$ \\
\hline
\end{tabular}


Table 5. - Computation of well protection zones for well fields - Continued

\begin{tabular}{|c|c|c|c|c|c|c|c|c|c|c|c|c|c|}
\hline $\begin{array}{l}\text { Well } \\
\text { name }\end{array}$ & $\begin{array}{c}\text { Cons. } \\
\text { use } \\
\text { permit }\end{array}$ & Lat. & Long. & Sec. & Tw. & ${ }_{\text {RE. }}^{\text {Rng }}$ & $\begin{array}{l}\text { Av.Q } \\
(\mathrm{sal} / \mathrm{d})\end{array}$ & $\begin{array}{l}\text { WoII } \\
\text { depth } \\
\text { (ft) }\end{array}$ & $\begin{array}{l}\text { FI } \\
(s e a \\
\text { lev. }\end{array}$ & $\begin{array}{l}\text { Isd } \\
(s e a \\
\text { lev. })\end{array}$ & $\begin{array}{c}h \\
\text { (ft) }\end{array}$ & $\begin{array}{c}r \\
\text { (ft) }\end{array}$ & $\begin{array}{l}\text { Area } \\
\left(\mathrm{ft}^{2}\right)\end{array}$ \\
\hline \multicolumn{14}{|c|}{ Morris Bridse--Continued } \\
\hline $\begin{array}{l}158 \\
159 \\
160 \\
161 \\
162 \\
163 \\
164 \\
165\end{array}$ & $\begin{array}{l}0418000 \\
0418000 \\
0418000 \\
0418000 \\
0418000 \\
0418000 \\
0418000 \\
0418000\end{array}$ & $\begin{array}{l}280727 \\
280659 \\
280628 \\
280610 \\
280626 \\
280615 \\
280629 \\
280643\end{array}$ & $\begin{array}{l}821846 \\
822051 \\
822059 \\
822107 \\
822008 \\
821952 \\
821931 \\
821924\end{array}$ & $\begin{array}{l}21 \\
19 \\
30 \\
30 \\
29 \\
29 \\
29 \\
29\end{array}$ & $\begin{array}{l}27 \\
27 \\
27 \\
27 \\
27 \\
27 \\
27 \\
27\end{array}$ & $\begin{array}{l}20 \\
20 \\
20 \\
20 \\
20 \\
20 \\
20 \\
20\end{array}$ & $\begin{array}{l}1,100,000 \\
1,100,000 \\
1,100,000 \\
1,100,000 \\
1,100,000 \\
1,100,000 \\
1,100,000 \\
1,100,000\end{array}$ & $\begin{array}{l}603 \\
553 \\
614 \\
542 \\
608 \\
616 \\
617 \\
620\end{array}$ & $\begin{array}{r}20 \\
0 \\
0 \\
0 \\
0 \\
0 \\
0 \\
10\end{array}$ & $\begin{array}{l}42 \\
39 \\
35 \\
34 \\
36 \\
34 \\
34 \\
32\end{array}$ & $\begin{array}{l}581 \\
514 \\
579 \\
508 \\
572 \\
582 \\
583 \\
598\end{array}$ & $\begin{array}{l}1,710 \\
1,820 \\
1,720 \\
1,830 \\
1,730 \\
1,710 \\
1,710 \\
1,690\end{array}$ & $\begin{array}{r}9,240,000 \\
10,440,000 \\
9,270,000 \\
10,570,000 \\
9,380,000 \\
9,220,000 \\
9,210,000 \\
8,980,000\end{array}$ \\
\hline $\begin{array}{l}166 \\
167 \\
168 \\
169\end{array}$ & $\begin{array}{l}0418000 \\
0418000 \\
0418000 \\
0418000\end{array}$ & $\begin{array}{l}280652 \\
280647 \\
280655 \\
280711\end{array}$ & $\begin{array}{l}821859 \\
821817 \\
821835 \\
821819\end{array}$ & $\begin{array}{l}21 \\
21 \\
21 \\
21\end{array}$ & $\begin{array}{l}27 \\
27 \\
27 \\
27\end{array}$ & $\begin{array}{l}20 \\
20 \\
20 \\
20\end{array}$ & $\begin{array}{l}1,200,000 \\
1,100,000 \\
1,100,000 \\
1,100,000\end{array}$ & $\begin{array}{l}599 \\
590 \\
617 \\
607\end{array}$ & $\begin{array}{r}0 \\
10 \\
10 \\
10\end{array}$ & $\begin{array}{l}35 \\
35 \\
38 \\
35\end{array}$ & $\begin{array}{l}564 \\
565 \\
589 \\
582\end{array}$ & $\begin{array}{l}1,820 \\
1,740 \\
1,700 \\
1,710\end{array}$ & $\begin{array}{r}10,380,000 \\
9,500,000 \\
9,110,000 \\
9,220,000\end{array}$ \\
\hline \multicolumn{14}{|c|}{ Sun City } \\
\hline $\begin{array}{l}\text { SUN1 } \\
\text { SUN2 } \\
\text { SUN3 } \\
\text { SUN4 } \\
\text { SUN5 } \\
\text { SUN6 } \\
\text { SUN8 }\end{array}$ & $\begin{array}{l}0435201 \\
0435201 \\
0435201 \\
0435201 \\
0435201 \\
0435201 \\
0435201\end{array}$ & $\begin{array}{l}274318 \\
274318 \\
274309 \\
274301 \\
274322 \\
274315 \\
274403\end{array}$ & $\begin{array}{l}822028 \\
822030 \\
822033 \\
822035 \\
822026 \\
822017 \\
822009\end{array}$ & $\begin{array}{r}6 \\
6 \\
7 \\
7 \\
6 \\
6 \\
31\end{array}$ & $\begin{array}{l}32 \\
32 \\
32 \\
32 \\
32 \\
32 \\
31\end{array}$ & $\begin{array}{l}20 \\
20 \\
20 \\
20 \\
20 \\
20 \\
20\end{array}$ & $\begin{array}{l}500,000 \\
500,000 \\
600,000 \\
700,000 \\
700,000 \\
500,000 \\
250,000\end{array}$ & $\begin{array}{l}829 \\
637 \\
704 \\
705 \\
900 \\
690 \\
500\end{array}$ & $\begin{array}{l}-90 \\
-90 \\
-90 \\
-90 \\
-90 \\
-90 \\
-80\end{array}$ & $\begin{array}{l}58 \\
58 \\
58 \\
58 \\
58 \\
65 \\
65\end{array}$ & $\begin{array}{l}681 \\
489 \\
556 \\
557 \\
752 \\
535 \\
355\end{array}$ & & \\
\hline SUN9 & 0435201 & 274443 & 822009 & 31 & 31 & 20 & 350,000 & 500 & -80 & 60 & 360 & & \\
\hline \multicolumn{14}{|c|}{ Riverview } \\
\hline $\begin{array}{l}\text { RWW1 } \\
\text { RWW2 } \\
\text { RWW3 } \\
\text { RWW4 } \\
\text { RWW5 } \\
\text { RWW6 } \\
\text { RWW7 } \\
\text { RWW8 }\end{array}$ & $\begin{array}{l}0435201 \\
0435201 \\
0435201 \\
0435201 \\
0435201 \\
0435201 \\
0435201 \\
0435201\end{array}$ & $\begin{array}{l}274907 \\
274903 \\
274857 \\
274857 \\
274901 \\
274904 \\
274909 \\
274914\end{array}$ & $\begin{array}{l}821953 \\
821953 \\
821953 \\
821947 \\
821941 \\
821947 \\
821947 \\
821947\end{array}$ & $\begin{array}{l}5 \\
5 \\
5 \\
5 \\
5 \\
5 \\
5 \\
5\end{array}$ & $\begin{array}{l}31 \\
31 \\
31 \\
31 \\
31 \\
31 \\
31 \\
31\end{array}$ & $\begin{array}{l}20 \\
20 \\
20 \\
20 \\
20 \\
20 \\
20 \\
20\end{array}$ & $\begin{array}{l}350,000 \\
300,000 \\
250,000 \\
250,000 \\
300,000 \\
200,000 \\
200,000 \\
250,000\end{array}$ & $\begin{array}{l}600 \\
700 \\
600 \\
725 \\
600 \\
750 \\
600 \\
688\end{array}$ & $\begin{array}{l}-50 \\
-50 \\
-50 \\
-50 \\
-50 \\
-50 \\
-50 \\
-50\end{array}$ & $\begin{array}{l}57 \\
58 \\
58 \\
62 \\
62 \\
60 \\
59 \\
58\end{array}$ & $\begin{array}{l}493 \\
592 \\
492 \\
613 \\
488 \\
640 \\
491 \\
580\end{array}$ & & \\
\hline
\end{tabular}

Table 6. - Composite protection zones for well fields

\begin{tabular}{|c|c|c|c|}
\hline Well-field name & $\begin{array}{l}\text { Permitted } \\
\text { average daily } \\
\text { withdrawal } \\
\text { (gallons) }\end{array}$ & $\begin{array}{c}\text { Average } \\
\text { thickness of } \\
\text { penetration of } \\
\text { Upper Floridan } \\
\text { aquifer (feet) }\end{array}$ & $\begin{array}{c}\text { Area of } \\
\text { protection } \\
\text { (square } \\
\text { miles) }\end{array}$ \\
\hline \multicolumn{4}{|c|}{ Pasco County } \\
\hline $\begin{array}{l}\text { South Pasco } \\
\text { Cypress Creek } \\
\text { Cross Bar Ranch } \\
\text { Starkey }\end{array}$ & $\begin{array}{r}16,900,000 \\
30,000,000 \\
30,000,000 \\
8,000,000\end{array}$ & $\begin{array}{l}608 \\
669 \\
644 \\
601\end{array}$ & $\begin{array}{l}4.87 \\
7.85 \\
8.15 \\
2.33\end{array}$ \\
\hline \multicolumn{4}{|c|}{ Pinellas/Hillsborough Counties } \\
\hline Eldridge-Wilde & $35,244,000$ & 304 & 20.3 \\
\hline \multicolumn{4}{|c|}{ Hillsborough County } \\
\hline $\begin{array}{l}\text { Section } 21 \\
\text { Cosme-Odessa } \\
\text { Morris Bridge }\end{array}$ & $\begin{array}{l}13,000,000 \\
13,000,000 \\
15,500,000\end{array}$ & $\begin{array}{l}452 \\
276 \\
561\end{array}$ & $\begin{array}{l}5.03 \\
8.24 \\
4.84\end{array}$ \\
\hline
\end{tabular}


Table 7.-Composite protection zones for individual wells with overlapping zones of protection - Continued

\begin{tabular}{|c|c|c|c|c|c|c|c|c|c|c|}
\hline Well & $\begin{array}{c}\text { Cons. } \\
\text { use } \\
\text { permit }\end{array}$ & $\begin{array}{l}\text { Av.Q } \\
(\mathrm{gal} / \mathrm{d})\end{array}$ & $\begin{array}{l}\text { Well } \\
\text { depth } \\
\text { (ft) }\end{array}$ & $\begin{array}{l}\text { Well } \\
\text { name }\end{array}$ & $\stackrel{r}{(f t)}$ & $\begin{array}{l}\text { Area } \\
\left(f t^{2}\right)\end{array}$ & $\begin{array}{l}\text { Tot.Q } \\
(\mathrm{gel} / \mathrm{d})\end{array}$ & $\begin{array}{l}\text { Avg. } \\
\text { h } \\
\text { (ft) }\end{array}$ & $\begin{array}{c}\text { Total area } \\
\left(f t^{2}\right)\end{array}$ & Quad. \\
\hline \multicolumn{11}{|c|}{ Bernando County--Continued } \\
\hline $\begin{array}{l}00989 \\
00990 \\
00991 \\
00992\end{array}$ & $\begin{array}{l}0298302 \\
0298302 \\
0298302 \\
0298302\end{array}$ & $\begin{array}{l}335,000 \\
335,000 \\
335,000 \\
335,000\end{array}$ & $\begin{array}{l}320 \\
218 \\
275 \\
275\end{array}$ & $\begin{array}{l}\text { WH-1 } \\
\text { WH-2 } \\
\text { WH-3 } \\
\text { WH-4 }\end{array}$ & 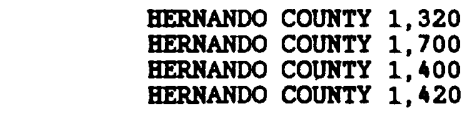 & $\begin{array}{l}5,450,000 \\
9,030,000 \\
6,170,000 \\
6,310,000\end{array}$ & $1,340,000$ & & $26,960,000$ & $\begin{array}{l}\text { Weeki- } \\
\text { wachee }\end{array}$ \\
\hline $\begin{array}{l}00536 \\
00537\end{array}$ & $\begin{array}{l}0340802 \\
0340802\end{array}$ & $\begin{array}{l}121,000 \\
121,000\end{array}$ & $\begin{array}{l}110 \\
400\end{array}$ & $\frac{1}{2}$ & $\begin{array}{l}\text { HERNANDO COUNTY } 1,900 \\
\text { HERNANDO COUNTY } \\
740\end{array}$ & $\begin{array}{r}11,350,000 \\
1,730,000\end{array}$ & 121,000 & 230 & * & $\begin{array}{l}\text { Brooks- } \\
\text { ville SE }\end{array}$ \\
\hline $\begin{array}{l}01085 \\
01089\end{array}$ & $\begin{array}{l}0484204 \\
0484204\end{array}$ & $\begin{array}{l}227,000 \\
397,000\end{array}$ & $\begin{array}{l}373 \\
350\end{array}$ & $\begin{array}{r}2 \\
11\end{array}$ & $\begin{array}{l}\text { SPRING BILL UTILITIES } 1,000 \\
\text { SPRING BILL UTILITIES } 1,360\end{array}$ & $\begin{array}{l}3,130,000 \\
5,850,000\end{array}$ & 624,000 & & $8,980,000$ & $\begin{array}{l}\text { Port } \\
\text { Richey N }\end{array}$ \\
\hline $\begin{array}{l}01087 \\
01095\end{array}$ & $\begin{array}{l}0484204 \\
0484204\end{array}$ & $\begin{array}{l}566,000 \\
325,000\end{array}$ & $\begin{array}{l}320 \\
300\end{array}$ & $\begin{array}{r}7 \\
20 P\end{array}$ & $\begin{array}{l}\text { SPRING GILL UTILITIES } 1,760 \\
\text { SPRING BILL UTILITIES } 1,380\end{array}$ & $\begin{array}{l}9,690,000 \\
5,980,000\end{array}$ & 891,000 & & $15,670,000$ & $\begin{array}{l}\text { Port } \\
\text { Richey N }\end{array}$ \\
\hline $\begin{array}{l}01088 \\
01091\end{array}$ & $\begin{array}{l}0484204 \\
0484204\end{array}$ & $\begin{array}{l}397,000 \\
227,000\end{array}$ & $\begin{array}{l}418 \\
484\end{array}$ & $\begin{array}{l}10 \\
13\end{array}$ & $\begin{array}{lr}\text { SPRING HILL UTILITIES } & 1,240 \\
\text { SPRING BILL UTILITIES } & 870\end{array}$ & $\begin{array}{l}4,860,000 \\
2,360,000\end{array}$ & 624,000 & & $7,240,000$ & $\begin{array}{l}\text { Port. } \\
\text { Richey N }\end{array}$ \\
\hline $\begin{array}{l}01090 \\
01096\end{array}$ & $\begin{array}{l}0484204 \\
0484204\end{array}$ & $\begin{array}{l}227,000 \\
227,000\end{array}$ & $\begin{array}{l}395 \\
300\end{array}$ & $\begin{aligned} 12 \\
21 P\end{aligned}$ & $\begin{array}{l}\text { SPRING HILL UTILITIES } 960 \\
\text { SPRING HILL UTILITIES } 1,110\end{array}$ & $\begin{array}{l}2,910,000 \\
3,890,000\end{array}$ & 454,000 & & $6,800,000$ & Port \\
\hline $\begin{array}{l}01092 \\
01094\end{array}$ & $\begin{array}{l}0484204 \\
0484204\end{array}$ & $\begin{array}{l}453,000 \\
325,000\end{array}$ & $\begin{array}{l}400 \\
400\end{array}$ & $\begin{aligned} 17 \\
19 P\end{aligned}$ & $\begin{array}{ll}\text { SPRING EILL UTILITIES } & 1,640 \\
\text { SPRING BILL UTILITIES } & 1,220\end{array}$ & $\begin{array}{l}6,500,000 \\
4,660,000\end{array}$ & 778,000 & & $11,160,000$ & $\begin{array}{l}\text { Weeki- } \\
\text { wachee }\end{array}$ \\
\hline $\begin{array}{l}01303 \\
01304\end{array}$ & $\begin{array}{l}0603901 \\
0603901\end{array}$ & $\begin{array}{l}450,000 \\
450,000\end{array}$ & $\begin{array}{l}200 \\
200\end{array}$ & $\begin{array}{l}2 \\
3\end{array}$ & $\begin{array}{ll}\text { U S B BOME CORP } & 1,980 \\
\text { U S HOME CORP } & 1,960\end{array}$ & $\begin{array}{l}12,340,000 \\
12,340,000\end{array}$ & 900,000 & & $24,680,000$ & $\begin{array}{l}\text { Port } \\
\text { Richey N }\end{array}$ \\
\hline $\begin{array}{l}01748 \\
01749\end{array}$ & $\begin{array}{l}0762700 \\
0762700\end{array}$ & $\begin{array}{l}200,000 \\
200,000\end{array}$ & $\begin{array}{l}593 \\
750\end{array}$ & $\begin{array}{l}1 \\
2\end{array}$ & $\begin{array}{l}\text { BROOKSVILLE, CITY OF } \\
\text { BROOKSVILLE, CITY OF }\end{array}$ & $\begin{array}{l}1,990,000 \\
1,510,000\end{array}$ & 400,000 & & $3,500,000$ & $\begin{array}{l}\text { Brooks- } \\
\text { ville }\end{array}$ \\
\hline $\begin{array}{l}01751 \\
01752\end{array}$ & $\begin{array}{l}0762700 \\
0762700\end{array}$ & $\begin{array}{l}250,000 \\
550,000\end{array}$ & $\begin{array}{l}750 \\
600\end{array}$ & $\begin{array}{l}4 \\
5\end{array}$ & $\begin{array}{l}\text { BROOKSVILLE, CITY OF } 800 \\
\text { BROOKSVILLE, CITY OF } 1,400\end{array}$ & $\begin{array}{l}2,030,000 \\
6,170,000\end{array}$ & 800,000 & & $8,200,000$ & $\begin{array}{l}\text { Brooks- } \\
\text { ville }\end{array}$ \\
\hline \multicolumn{11}{|c|}{ Pasco County } \\
\hline $\begin{array}{l}00434 \\
00435 \\
00436 \\
00437\end{array}$ & $\begin{array}{l}0297601 \\
0297601 \\
0297601 \\
0297601\end{array}$ & $\begin{array}{l}150,000 \\
150,000 \\
150,000 \\
200,000\end{array}$ & $\begin{array}{l}281 \\
267 \\
209 \\
200\end{array}$ & $\begin{array}{l}1 \\
2 \\
5 \\
7\end{array}$ & $\begin{array}{lr}\text { PASCO COUNTY } & 970 \\
\text { PASCO COUNTY } & 960 \\
\text { PASCO COUNTY } & 1,140 \\
\text { PASCO COUNTY } & 1,320\end{array}$ & $\begin{array}{l}2,930,000 \\
2,900,000 \\
4,070,000 \\
5,450,000\end{array}$ & 650,000 & & $15,410,000$ & Port \\
\hline $\begin{array}{l}00608 \\
00609\end{array}$ & $\begin{array}{l}0369203 \\
0369203\end{array}$ & $\begin{array}{l}112,000 \\
237,000\end{array}$ & $\begin{array}{l}230 \\
150\end{array}$ & $\frac{1}{2}$ & $\begin{array}{l}\text { PORT RICHEY, CITY OF } 910 \\
\text { PORT RICHEY, CITY OF } 1,680\end{array}$ & $\begin{array}{l}2,610,000 \\
8,830,000\end{array}$ & 349,000 & & $11,440,000$ & $\begin{array}{l}\text { Port } \\
\text { Richey }\end{array}$ \\
\hline $\begin{array}{l}00623 \\
00624\end{array}$ & $\begin{array}{l}0376101 \\
0376101\end{array}$ & $\begin{array}{l}435,000 \\
115,000\end{array}$ & $\begin{array}{l}350 \\
350\end{array}$ & $\begin{array}{l}101 \\
102\end{array}$ & $\begin{array}{l}\text { PASCO COUNTY } 1,410 \\
\text { PASCO COUNTY }\end{array}$ & $\begin{array}{l}6,220,000 \\
1,660,000\end{array}$ & 550,000 & & $7,880,000$ & Fivay \\
\hline $\begin{array}{l}00966 \\
00967 \\
00968 \\
00969 \\
00970 \\
00971 \\
00972 \\
00973 \\
00974\end{array}$ & $\begin{array}{l}0466902 \\
0466902 \\
0466902 \\
0466902 \\
0466902 \\
0466902 \\
0466902 \\
0466902 \\
0466902\end{array}$ & $\begin{array}{l}100,000 \\
100,000 \\
100,000 \\
100,000 \\
100,000 \\
100,000 \\
100,000 \\
100,000 \\
100,000\end{array}$ & $\begin{array}{r}100 \\
100 \\
100 \\
50 \\
100 \\
90 \\
105 \\
95 \\
93\end{array}$ & $\begin{array}{r}1 \\
3 \\
4 \\
5 \\
7 \\
11 \\
13 \\
14 \\
15\end{array}$ & $\begin{array}{l}\text { HUDSON WATER WORKS INC. } 1,370 \\
\text { HUDSON WATER WORKS INC. } 1,360 \\
\text { HUDSON WATER WORKS INC. } 1,370 \\
\text { HUDSON WATER WORKS INC. } 2,140 \\
\text { HUDSON WATER WORKS INC. } 1,400 \\
\text { HUDSON WATER WORKS INC. } 1,450 \\
\text { HUDSON WATER WORKS INC. } 1,400 \\
\text { HUDSON WATER WORKS INC. } 1,420 \\
\text { HUDSON WATER WORKS INC. } 1,420\end{array}$ & $\begin{array}{r}5,880,000 \\
5,610,000 \\
5,880,000 \\
14,350,000 \\
6,180,000 \\
6,590,000 \\
6,180,000 \\
6,340,000 \\
6,340,000\end{array}$ & 582,353 & 82 & $34,650,000$ & Port \\
\hline $\begin{array}{l}00975 \\
00976 \\
00977 \\
00978 \\
00979 \\
00980 \\
00981 \\
00982\end{array}$ & $\begin{array}{l}0466902 \\
0466902 \\
0466902 \\
0466902 \\
0466902 \\
0466902 \\
0466902 \\
0466902\end{array}$ & $\begin{array}{l}100,000 \\
100,000 \\
100,000 \\
100,000 \\
100,000 \\
100,000 \\
100,000 \\
100,000\end{array}$ & $\begin{array}{l}100 \\
100 \\
100 \\
100 \\
100 \\
100 \\
100 \\
100\end{array}$ & $\begin{array}{l}16 \\
17 \\
18 \\
19 \\
20 \\
21 \\
22 \\
23\end{array}$ & $\begin{array}{l}\text { HUDSON WATER WORKS INC. } 1,510 \\
\text { HUDSON WATER WORKS INC. } 1,530 \\
\text { HUDSON WATER WORKS INC. } 1,410 \\
\text { HUDSON WATER WORKS INC. } 1,510 \\
\text { HUDSON WATER WORKS INC. } 1,410 \\
\text { HUDSON WATER WORKS INC. } 1,370 \\
\text { HUDSON WATER WORKS INC. } 1,470 \\
\text { HUDSON WATER WORKS INC. } 1,460\end{array}$ & $\begin{array}{l}7,180,000 \\
7,390,000 \\
6,260,000 \\
7,180,000 \\
6,260,000 \\
5,880,000 \\
6,780,000 \\
6,680,000\end{array}$ & 517,647 & 65 & $38,660,000$ & Aripeka \\
\hline \multirow[t]{2}{*}{$\begin{array}{l}01672 \\
01673\end{array}$} & $\begin{array}{l}0728200 \\
0728200\end{array}$ & $\begin{array}{l}375,000 \\
375,000\end{array}$ & $\begin{array}{l}133 \\
133\end{array}$ & $\begin{array}{l}P W-3 \\
P W-4\end{array}$ & $\begin{array}{ll}\text { PASCO COUNTY } & 2,130 \\
\text { PASCO COUNTY } & 2,140\end{array}$ & $\begin{array}{l}14,300,000 \\
14,410,000\end{array}$ & 950,000 & & $28,640,000$ & Port \\
\hline & & & & & HUDSON + PASCO COUNTY & & $2,100,000$ & & $102,150,000$ & \\
\hline 01001 & 0473401 & 440,000 & 300 & W-5 & NEW PORT RICHEY, CITY OF 1,540 & $7,400,000$ & 440,000 & & $\star \star$ & \\
\hline
\end{tabular}


Table 7.-Composite protection zones for individual wells with overlapping zones of protection - Continued

\begin{tabular}{|c|c|c|c|c|c|c|c|c|c|}
\hline Well & $\begin{array}{l}\text { Cons. } \\
\text { use } \\
\text { permit }\end{array}$ & $\begin{array}{c}\text { Av.Q } \\
(\mathrm{gal} / \mathrm{d})\end{array}$ & $\begin{array}{l}\text { Well } \\
\text { depth Well } \\
\text { (ft) name }\end{array}$ & Orner & $\stackrel{r}{(f t)}$ & $\begin{array}{l}\text { Area } \\
\left(\mathrm{ft}^{2}\right)\end{array}$ & $\begin{array}{l}\text { Tot.Q } \\
(\mathrm{gal} / \mathrm{d})\end{array}$ & $\begin{array}{l}\text { Avg. Total area } \\
h(f t) \quad\left(\mathrm{ft}^{2}\right) \\
\text { (ft) }\end{array}$ & Quad. \\
\hline
\end{tabular}

\section{Leaky confined area}

Pasco County

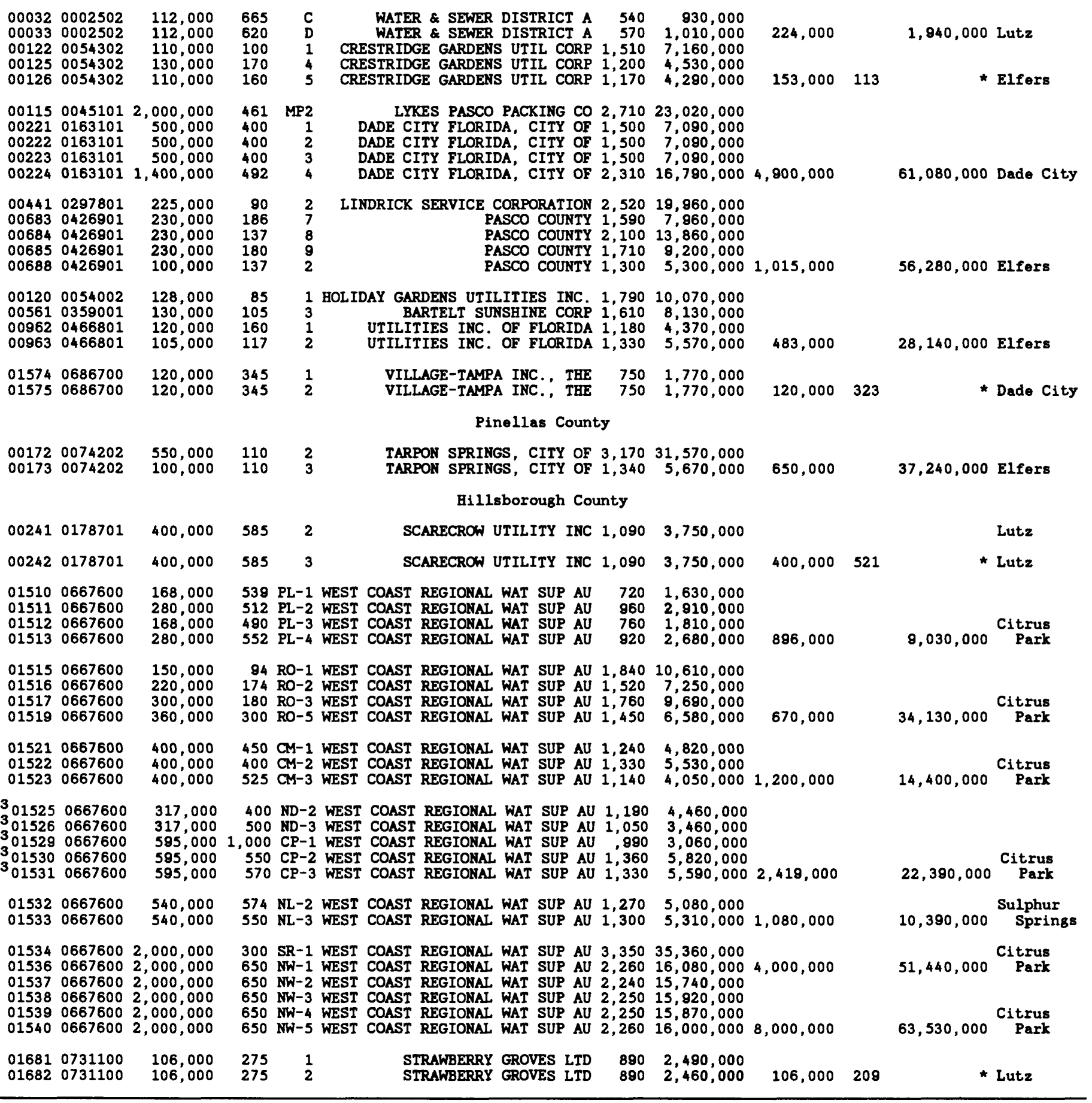

${ }^{3}$ This composite protection zone is show in figure 9. 


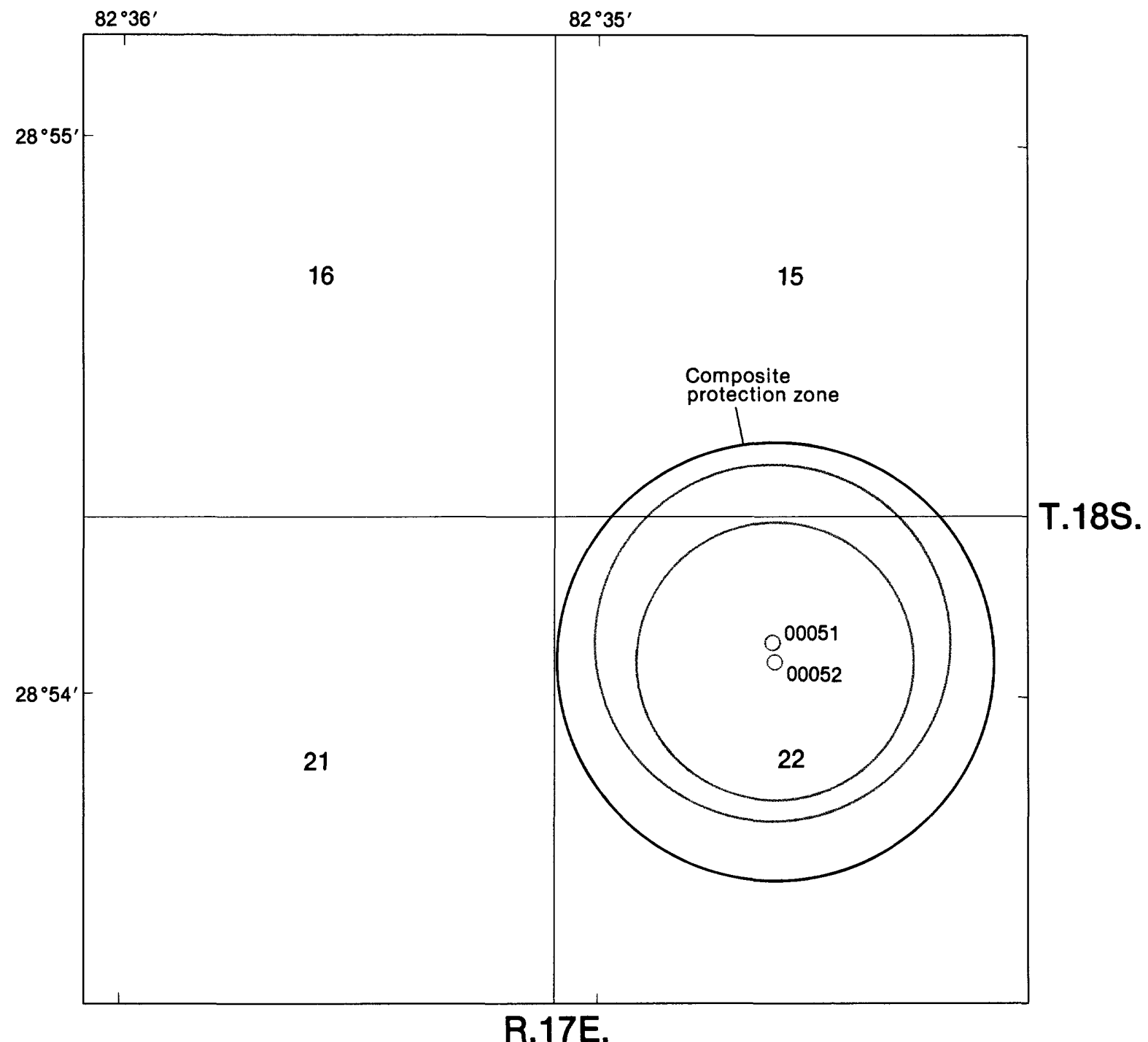

\section{EXPLANATION}

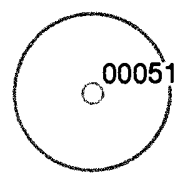

OUTER CIRCLE IS PROTECTION ZONE FOR PUBLIC SUPPLY WELL LOCATED IN CENTER OF CIRCLE. NUMBER IS $\begin{array}{ccccccc}\stackrel{0}{0} & 1,000 & 2,000 & 3,000 & 4,000 & 5,000 & \text { FEET } \\ 0 & & 500 & 1,000 & & 1,500 & \text { METERS }\end{array}$ WELL NUMBER IN TABLES.

Figure 7. - Composite protection zones for wells of the city of Crystal River, Citrus County. 


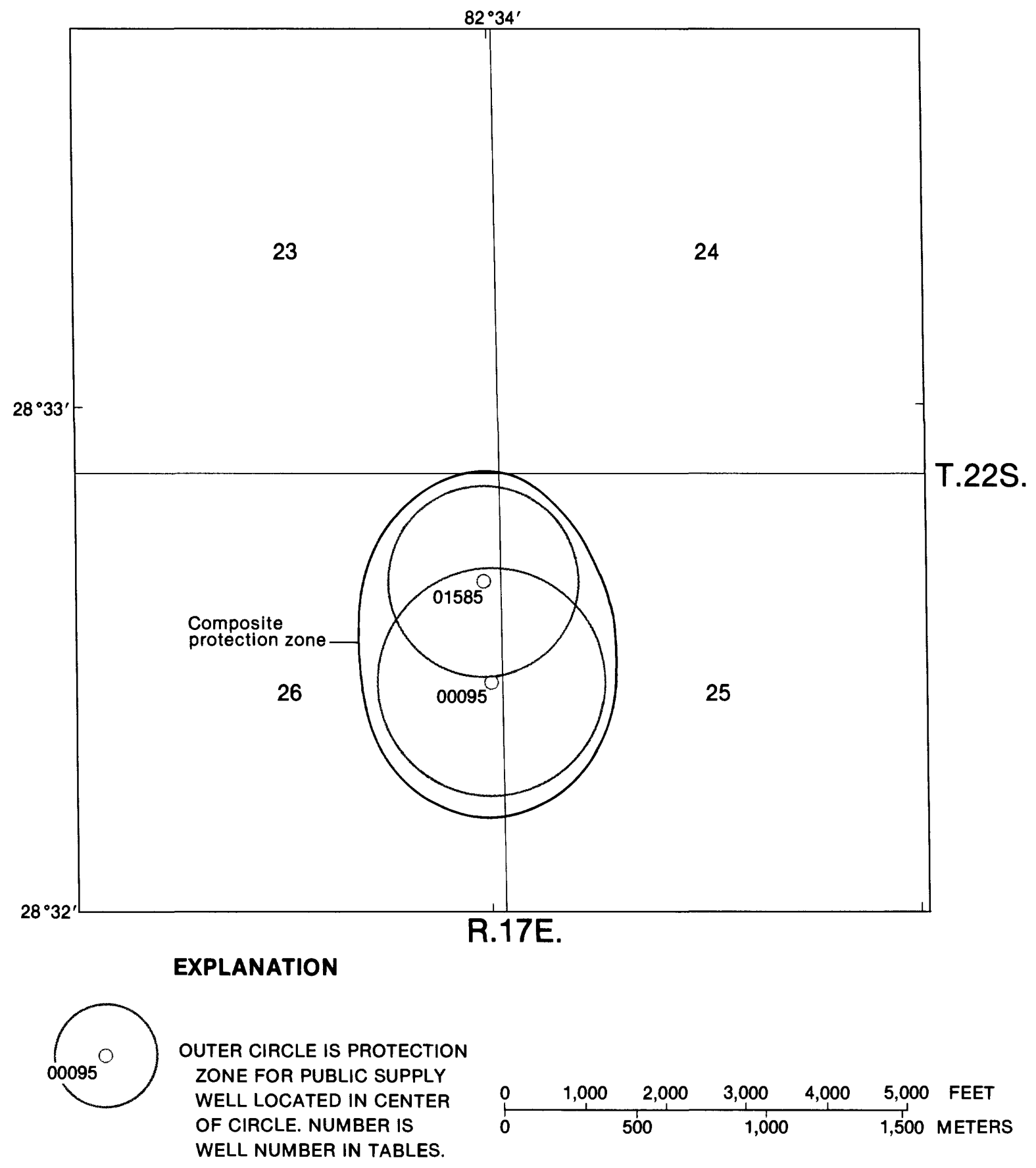

Figure 8. - Composite protection zones for wells of the Royal Palm Beach Colony, Inc., Hernando County. 


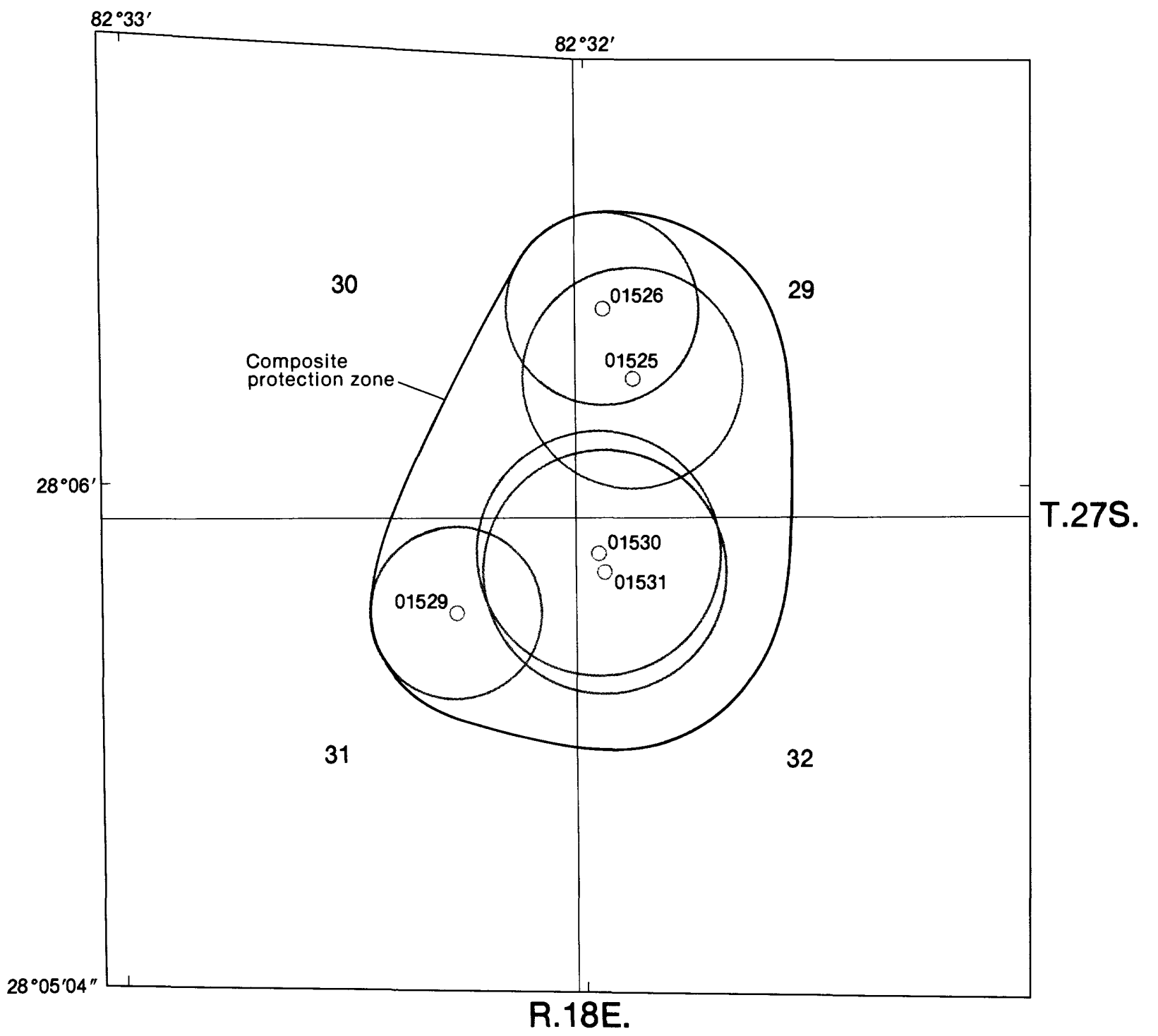

EXPLANATION

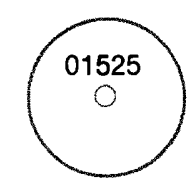

OUTER CIRCLE IS PROTECTION ZONE FOR PUBLIC SUPPLY WELL LOCATED IN CENTER OF CIRCLE. NUMBER IS \begin{tabular}{ccccccc}
$\stackrel{0}{1,000}$ & 2,000 & 3,000 & 4,000 & 5,000 & FEET \\
0 & \multicolumn{1}{l}{$1,1,1$} & 1,000 & & 1,500 & METERS
\end{tabular} WELL NUMBER IN TABLES.

Figure 9. - Composite protection zones for some wells of the West Coast Regional Water Supply Authority, Hillsborough County. 


\section{EVALUATION OF METHODOLOGY FOR DELINEATING PROTECTION ZONES}

Basis of the Volumetric Equation

The volumetric equation used in delineating the protection zones is based on a simplification of the ground-water flowfield around a pumping well. The flowfield is conceptualized as one of uniform radial flow toward the pumping well emanating from distances far beyond the anticipated "protection-zone boundary." Only lateral flow is presumed and there are no incremental gains from storage, recharge, or leakage as the pumping well is approached. Accordingly, invoking the law of conservation of mass requires that the volume of water pumped from the well in a specified time be replaced by an equal volume flowing through a cylindrical face of the aquifer at a distance " $r$ " from the well such that the volume of aquifer circumscribed by the cylinder contains the volume of water pumped. The model further presumes that a potential contaminant passing through the cylindrical face would be transported toward the well under advective "plug-flow" conditions only. Thus, the time required for a potential contaminant to enter the well from some distance " $r$ " in the aquifer is simply a function of the amount of water in the cylinder of aquifer around the well that must be displaced.

Although appearing to be devoid of variables such as hydraulic conductivity or velocity and hydraulic gradient, the volumetric equation is derivable, as suggested by Mark T. Stewart (University of South Florida, written commun., December 16, 1986), from the radial form of Darcy's law:

$$
\mathbf{Q}=\mathrm{K} 2 \pi \mathrm{rhi}
$$

where

$\mathbf{Q}=$ rate of discharge,

$\mathbf{K}=$ hydraulic conductivity,

$\mathbf{r}=$ radial distance,

$\mathbf{h}=$ aquifer thickness, and

$\mathrm{i}=$ hydraulic gradient.

Recognizing that average particle velocity, $\bar{v}$, times porosity, $\mathbf{n}$, is equal to $\mathrm{Ki}$, then through substitution and rearrangement,

$\overline{\mathbf{v}}=\frac{\mathrm{Q}}{2 \pi \mathrm{rhn}}$,

and substitution of $r / t$, where $t=$ time, for $\bar{v}$, differentiation of $r$ with respect to $t$, and rearrangement yields

$\mathbf{r d r}=\frac{\mathrm{Q}}{2 \pi \mathrm{hn}} \mathrm{dt}$ and integrating from $t_{1}$ to $t_{2}$;

$$
\int_{r_{1}}^{r_{2}} r d r=\frac{Q}{2 \pi h n} \int_{t_{1}}^{t_{2}} d t
$$

results in $\frac{r_{2}^{2}}{2}-\frac{r_{1}^{2}}{2}=\frac{Q}{2 \pi h n}\left(t_{2}-t_{1}\right)$,

and when $t_{1}=0, r_{1}=0$,

gives $\frac{\mathbf{r}^{2}}{2}=\frac{\mathrm{Qt}}{2 \pi \mathrm{hn}}$

or $\mathbf{r}=\left[\frac{\mathrm{Qt}}{\pi \mathrm{hn}}\right]^{1 / 2}$

\section{Hydrologic Factors Influencing Applicability}

\section{Potentiometric-Surface Slope}

Superposition of the drawdown from a pumping well producing from an aquifer that has a sloping potentiometric surface results in a nonradially symmetric flowfield around the well. As described by Todd (1980, p. 122), the boundary of the contributing area extends downgradient to a stagnation point beyond which water is not drawn toward the pumping well. However, in the upgradient direction, the contributing area extends to a greater distance. The greater the slope of the potentiometric surface, the closer is the stagnation point in the downgradient direction and the greater the flowfield departs from being radially symmetrical.

As a result of the distortion from a radially symmetrical flowfield, application of the volumetric equation to a well pumping from an aquifer with a sloping potentiometric surface delineates a protection zone that may be overprotective in the downgradient direction and underprotective in the upgradient direction. The degree to which inappropriate areas are included within the delineated protection zones can be assessed by considering a range of gradients typical of the slope of the potentiometric surface in west-central Florida. From potentiometric-surface maps of the Upper Floridan aquifer (Barr, 1985b; Barr and Lewelling, 1986), it is seen that the hydraulic gradient varies from near zero to a maximum of about 0.001 . Table 8 compares the downgradient distance to the stagnation point with the radial distance calculated from the volumetric equation for typical pumping rates, aquifer characteristics, and gradient extremes. For example, the stagnation point where the transmissivity is 25,000 $\mathrm{ft}^{2} / \mathrm{d}$ and the horizontal gradient is 0.0001 is 8,500 feet 
Table 8. - Comparison of downgradient distance to stagnation point with radial distance calculated from volumetric equation

$$
\left[\mathrm{ft}^{2} / \mathrm{d}=\text { foot squared per day; Mgal/d = million gallons per day }\right]
$$

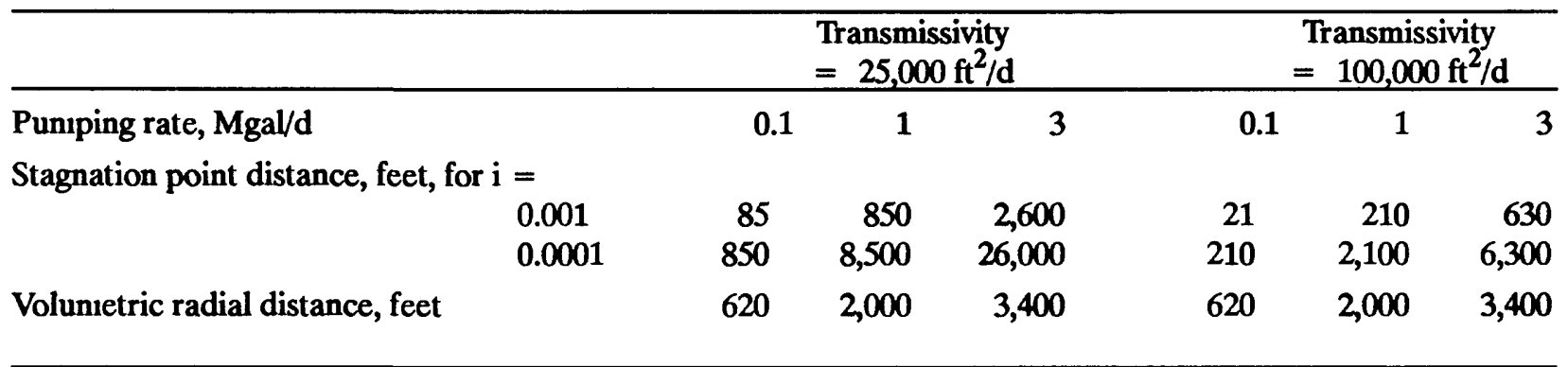

Note: Stagnation point distance computed from $\chi_{\mathrm{L}}=-\frac{\mathrm{Q}}{2 \pi \mathrm{Kmi}}$ (Todd, 1980, p. 123)

where

$\chi_{\mathrm{L}}=$ downgradient stagnation point distance from well,

$\mathbf{Q}=$ puniping rate,

$\mathbf{K}=$ aquifer hydraulic conductivity,

$\mathbf{m}=$ aquifer thickness, and

$\mathrm{i}=$ hydraulic gradient.

Volunetric radial distance computed from equation 4 with $h=400$ feet and $n=0.05$.

downgradient of a well pumping $1 \mathrm{Mgal} / \mathrm{d}$. Because the stagnation point is farther from the well than is the radial distance $(2,000$ feet) calculated from the volunetric equation for typical conditions in the area, the calculated protection zone lies fully within the contributing area. In this case, the calculated zone is only slightly overprotective on the downgradient side. This results because the head gradient at any given radius from the well will be less on the downgradient side than on the upgradient side when the sloping potentiometric surface is considered. In cases where the stagnation point is closer to the well than the radial distance from the volunietric equation, the computed protection zone is more significantly overprotecting the area downgradient of the well, because water on the downgradient side of the stagnation point cannot flow to the well.

\section{Boundaries}

The conceptual model used as the basis for the volunetric equation assumes that the aquifer extends from the well to be protected in all directions to distances far beyond the boundaries of the protection zone and is not intersected by hydraulic boundaries. For leaky confined aquifers, that assumption is valid because the aquifer is isolated hydraulically to some degree from surface streams and lakes that would function as recharge boundaries. For unconfined aquifers, the presence of streams, lakes, or canals in the vicinity of a public-supply well would distort the flowfield around the well. If the surface-water source were in good hydraulic cominunication with the unconfined aquifer, it could function as a fully effective boundary that prevents the flowfield produced by the pumping well from spreading beyond the boundary. Therefore, if the protection zone calculated with the volumetric formula extends beyond the surface-water body, that part of the zone that falls beyond may be regarded as unnecessary protection. On the other hand, that part of the zone that falls beyond, if not regulated, could contribute contaminants to the surface-water body and thence indirectly to the publicsupply well through induced infiltration.

\section{Porosity Variation}

Effective porosity is assumed to be uniform around the puniping well and not to vary from site to site. The radius calculated from the volumetric equation varies inversely with the square root of effective porosity. Accordingly, a 100 percent increase in the assigned effective porosity - say from 0.05 to 0.10 -results in a calculated radius that is 71 percent of the radius using 0.05 . A 200 percent increase in effective porosity would result in a 42 percent decrease in radius. 
Effective porosity refers to the amount of interconnected pore space available for fluid transmission (Lohman and others, 1972, p. 10). Some aquifers are characterized by diffuse flow in which water moves more or less uniformly throughout the interconnected pores distributed throughout the mass of the rock. Unconsolidated sand and sand and gravel aquifers fall into this category as does the Biscayne aquifer in general. Some consolidated rock aquifers, such as the Floridan, are characterized by conduit flow along irregularly distributed solution enlarged fractures in combination with diffuse flow through the much more uniformly distributed interconnected pores. In these aquifers, water tends to travel fastest through the fracture openings and thus contaminant movement is dominated by the porosity associated with the fracture openings, as shown schematically by Freeze and Cherry (1979, p. 411) and described by Gilham and others (1983, p. 23). In contrast, the amount of porosity associated with fracture openings is much less than that of the pores; thus the effective porosity value of 0.05 assigned to the Floridan in the FDER regulation is much less than that assigned to the other aquifers that possess diffuse-flow characteristics. However, the assigned porosity value is simply a judgment and is not based on specific measurements of fracture porosity, because fracture porosity is variable and extremely difficult to measure.

\section{Thickness Determination}

The thickness-of-aquifer value used in the volumetric equation is prescribed as the distance between the top of the aquifer and the bottom of the well. As with porosity, the calculated radius of the protection zone varies inversely with the square root of the aquifer thickness. For example, if a well penetrated the Floridan aquifer system in the west-central Florida area to a depth of 400 feet, a not uncominon depth, but the overall thickness of the aquifer were 25 to 50 percent greater, then the calculated radius would be 12 to 25 percent greater from what it would be if the full aquifer thickness were used in the calculation.

\section{SUMMARY}

Numerous public-supply wells in west-central Florida tap the Floridan aquifer system. Because the Floridan is at or near land surface and unconfined to poorly confined over most of the area of northern Hillsborough and Pinellas Counties and Pasco, Hernando, and Citrus Counties, it is vulnerable to contamination from surface sources. A recent regulation of the Florida Department of Environmental Regulation (1987) calls for the delineation of protection zones around public-supply wells that tap such vulnerable aquifers. This report evaluates the conditions of confinement of the Upper Floridan aquifer at each of the public-supply well sites and it defines protection zones where required in accordance with the FDER regulation.

Vulnerability was assessed by delineating areas where the Floridan aquifer system is unconfined, leaky confined, or confined. Leaky confined areas were evaluated to determine vertical travel time through overlying material to the Floridan. Protection zones were calculated for wells and well fields of greater than 100,000 gallons per day permitted use in unconfined areas and in leaky confined areas with less than 5-year vertical travel time from the water table to the top of the Floridan. Protection zones were delineated using a volumetric displacement model derivable from Darcy's Law. Where zones of individual wells did not overlap, radii of circles of appropriate area around individual wells were tabulated. Where zones overlapped, composite areas were determined and composite protection zones were delineated in shapes that were appropriate for each configuration of wells with overlapping zones.

The report evaluates the effects of various hydrogeologic factors on the size and/or shape of the protection zones delineated in accordance with the FDER regulations. The natural slope of the potentiometric surface, hydrologic boundaries, porosity variations, and differences in total aquifer thickness from that penetrated by the public-supply well all cause the delineated protection zone to depart, in varying degree, from the actual 5-year travel-time threshold boundary.

\section{SELECTED REFERENCES}

Anderson, Warren, and Laughlin, C.P., 1982, Geohydrology of the Floridan aquifer in the Withlacoochee River basin of the Southwest Florida Water Management District: U.S. Geological Survey Water-Resources Investigations Report 82-331, 2 sheets.

Barr, G.L., 1982, Water levels in selected well fields and in west-central Florida, May 1982: U.S. Geological Survey Open-File Report 82-867, 2 sheets.

- - 1985a, Potentiometric surface of the Upper Floridan aquifer, west-central Florida, May 1985: U.S. Geological Survey Open-File Report 85482, 1 sheet.

- - 1985b, Potentiometric surface of the Upper Floridan aquifer, west-central Florida, September 1985: U.S. Geological Survey Open-File Report 85-679, 1 sheet. 
Barr, G.L., and Lewelling, B.R., 1986, Potentiometric surface of the Upper Floridan aquifer, westcentral Florida, May 1986: U.S. Geological Survey Open-File Report 86-409, 1 sheet.

Barr, G.L., and Schiner, G.R., 1982, Potentiometric surface of the Floridan aquifer, Southwest Florida Water Management District, May 1982: U.S. Geological Survey Open-File Report 82697, 1 sheet.

Bredehoeft, J.D., Papadopulos, I.S., and Stewart, J.W., 1965, Hydrologic effects of pumping in northwest Hillsborough County, Florida: U.S. Geological Survey Open-File Report 65001, 24 p.

Buono, Anthony, and Rutledge, A.T., 1979, Configuration of the top of the Floridan aquifer, Southwest Florida Water Management District and adjacent areas: U.S. Geological Survey WaterResources Investigations/Open-File Report 78-34, 1 sheet.

Buono, Anthony, Spechler, R.M., Barr, G.L., and Wolansky, R.M., 1979, Generalized thickness of the confining bed overlying the Floridan aquifer, Southwest Florida Water Management District: U.S. Geological Survey Water-Resources Investigations/Open-File Report 79-1171, 1 sheet.

Bush, P.W., 1982, Predevelopment flow in the Tertiary limestone aquifer system, southeastern United States: A regional analysis from digital modeling: U.S. Geological Survey Water-Resources Investigations Report 82-905, $41 \mathrm{p}$.

Causseaux, K.W., 1985, The surficial aquifer in Pinellas County, Florida: U.S. Geological Survey Water-Resources Investigations 84-4289, 26 p.

Cherry, R.N., Stewart, J.W., and Mann, J.A., 1970, General hydrology of the Middle Gulf area, Florida: Florida Bureau of Geology Report of Investigations $56,96 \mathrm{p}$.

Cooper, H.H., Jr., and Jacob, C.E., 1946, A generalized graphical method for evaluating formation constants and summarizing well-field history: American Geophysical Union Transactions, v. 27, no. 4 , p. 526-534.

Duerr, A.D., 1974, Hydrologic data from wells in southwest Hillsborough County, Florida: U.S. Geological Survey Open-File Report FL-74031, $53 \mathrm{p}$.

Faulkner, G.L., 1972, Mid-1971 ground-water conditions at Yankeetown well field, Levy County, Florida: U.S. Geological Survey Open-File Report FL-72001, 18 p.
- - 1973a, Geohydrology of the Cross-Florida Barge Canal area with special reference to the Ocala vicinity: U.S. Geological Survey WaterResources Investigations 1-73, $117 \mathrm{p}$.

- $-1973 b$, Ground-water conditions in the lower Withlacoochee River-Cross Florida Barge Canal complex area: U.S. Geological Survey WaterResources Investigations Report 4-72, $31 \mathrm{p}$.

Florida Department of Environmental Regulation, 1987, Water quality standards, Chapter 17.3 in Florida Administrative Code.

Freeze, R.A., and Cherry, J.A., 1979, Groundwater: Englewood Cliffs, NJ., Prentice-Hall, Inc., 604 p.

Fretwell, J.D., 1983, Ground-water resources of coastal Citrus, Hernando, and southwestern Levy Counties, Florida: U.S. Geological Survey Water-Resources Investigations Report 83-4079, $87 \mathrm{p}$.

Gilham, R.W., Robin, M.J.L., Barkey, J.F., and Cherry, J.A., 1983, Groundwater monitoring and sample bias: American Petroleum Institute Publication 4367, $206 \mathrm{p}$.

Grubb, H.F., 1978, Potential for downward leakage to the Floridan aquifer, Green Swamp area, central Florida, U.S. Geological Survey Water-Resources Investigations Report 77-71, 1 sheet.

Grubb, H.F., and Rutledge, A.T., 1979, Long-term water supply potential, Green Swamp area, Florida: U.S. Geological Survey Water-Resources Investigations Report 78-99, 76 p.

Heath, R.C., and Smith, P.C., 1954, Ground-water resources of Pinellas County, Florida: Florida Geological Survey Report of Investigations 12, $139 \mathrm{p}$.

Hutchinson, C.B., 1978, Appraisal of shallow groundwater resources and management alternatives in the upper Peace and eastern Alafia River basins, Florida: U.S. Geological Survey Water-Resources Investigations 77-124, $57 \mathrm{p}$.

- - 1984, Hydrogeology of well-field areas near Tampa, Florida, phase 2-Development and documentation of a quasi-three dimensional finite-difference model for simulation of steadystate ground-water flow: U.S. Geological Survey Water-Resources Investigations Report 84-4002, $174 \mathrm{p}$.

- - - 1985, Hydrogeology of the Cross Bar Ranch well-field area and projected impact of pumping, Pasco County, Florida: U.S. Geological Survey Water-Resources Investigations Report 85-4001, 89 p. 
Hutchinson, C.B., Johnson, D.M., and Gerhart, J.M., 1981, Hydrogeology of well-field areas near Tampa, Florida, phase 1-Developments and documentation of a two-dimensional finite-difference model for simulation of steady-state ground-water flow: U.S. Geological Survey Open-File Report 81-630, 129 p.

Jacob, C.E., 1945, Partial penetration of pumping well, adjustments for: U.S. Geological Survey open-file report, $7 \mathrm{p}$.

Johnston, R.H., Krause, R.E., Meyer, F.W., Ryder, P.D., Tibbals, C.H., and Hunn, J.D., 1980, Estimated potentiometric surface for the Tertiary limestone aquifer system, southeastern United States, prior to development: U.S. Geological Survey Open-File Report 80-406, 1 sheet.

Kozeny, J., 1933, Theorie und Berechnung der Brunnen: Wasserkraft und Wasserwirtschaft, v. 28, p. 101.

Lohman, S.W., 1972, Ground-water hydraulics: U.S. Geological Survey Professional Paper 708, 70 p.

Lohman, S.W., and others, 1972, Definitions of selected ground-water terms-Revisions and conceptual refinements: U.S. Geological Survey Water-Supply Paper 1988, 21 p.

Menke, C.G., Meredith, E.W , and Wetterhall, W.S., 1961, Water resources of Hillsborough County, Florida: Florida Geological Survey Report of Investigations $25,101 \mathrm{p}$.

- -1964 , Water-resources records of Hillsborough County, Florida: Florida Geological Survey Information Circular 44, 95 p.

Miller, J.A., 1982, Geology and configuration of the top of the Tertiary limestone aquifer system, southeastern United States: U.S. Geological Survey Open-File Report 81-1178, 1 sheet.

- - 1986, Hydrogeologic framework of the Floridan aquifer system in Florida and in parts of Georgia, Alabama, and South Carolina: U.S. Geological Survey Professional Paper 1403-B, 9 p.

Miller, R.A., Anderson, Warren, Navoy, A.S., Smoot, J.L., and Belles, R.G., 1981, Water-resources information for the Withlacoochee River region, west-central Florida: U.S. Geological Survey Water-Resources Investigations Report 81-11, $130 \mathrm{p}$.

Parker, G.G., Ferguson, G.E., Love, S.K., and others, 1955, Water resources of southeastern Florida: U.S. Geological Survey Water-Supply Paper $1255,965 \mathrm{p}$.
Peek, H.M., 1958, Record of wells in Manatee County, Florida: Florida Geological Survey Information Circular 19, $199 \mathrm{p}$.

- $-1959 \mathrm{a}$, The artesian water of the Ruskin area of Hillsborough County, Florida: Florida Geological Survey Report of Investigations 21, $96 \mathrm{p}$.

- $-1959 \mathrm{~b}$, Record of wells in the Ruskin area, Hillsborough County, Florida: Florida Geological Survey Information Circular 22, 85 p.

Pride, R.W., Meyer, F.W., and Cherry, R.N., 1961, Interim report on the hydrologic features of the Green Swamp area, in central Florida: Florida Geological Survey Information Circular 26,96 p.

- - 1966, Hydrology of the Green Swamp area in central Florida: Florida Geological Survey Report of Investigations 42, $137 \mathrm{p}$.

Robertson, A.F., and Mallory, J.J., 1977, A digital model of the Floridan aquifer north of Tampa, Florida: U.S. Geological Survey Water-Resources Investigations Report 77-64, 29 p.

Ryder, P.D., 1978, Model evaluation of the Cypress Creek well field in west-central Florida: U.S. Geological Survey Water-Resources Investigations Report 78-79, 68 p.

- -1981 , Digital model of predevelopment flow in the Tertiary hinestone (Floridan) aquifer system in west-central Florida: U.S. Geological Survey Water-Resources Investigations 81-54, $61 \mathrm{p}$.

- - 1985, Hydrology of the Floridan aquifer system in west-central Florida: U.S. Geological Survey Professional Paper 1403-F, 63 p.

Ryder, P.D., Johnson, D.M., and Gerhart, J.M., 1980, Model evaluation of the hydrogeology of the Morris Bridge well field and vicinity in westcentral Florida: U.S. Geological Survey WaterResources Investigations Report 80-29, 92 p.

Ryder, P.D., and Mills, L.R., 1977, Water table in the surficial aquifer and potentiometric surface of the Floridan aquifer in selected well fields, westcentral Florida, September 1976: U.S. Geological Survey Open-File Report 77-642, 4 sheets.

Sinclair, W.C., 1974, Hydrogeologic characteristics of the surficial aquifer in northwest Hillsborough County, Florida: Florida Bureau of Geology Information Circular 86, $98 \mathrm{p}$.

- - 1977, Experimental study of artificial recharge alternatives in northwest Hillsborough County, Florida: U.S. Geological Survey Water-Resources Investigations Report 77-13, 52 p. 
Sinclair, W.C., Stewart, J.W., Knutilla, R.L., Gilboy, A.E., and Miller, R.L., 1985, Types, features, and occurrence of sinkholes in the karst of westcentral Florida: U.S. Geological Survey WaterResources Investigations Report 85-4126, 81 p.

Southeastern Geological Society Ad Hoc Committee on Florida Hydrostratigraphic Unit Definition, 1986, Hydrogeological units of Florida: Florida Geological Survey, Special Publication No. 28, 9 p.

Stewart, J.W., 1968, Hydrologic effects of pumping from the Floridan aquifer in northwest Hillsborough, northeast Pinellas, and Southwest Pasco Counties, Florida: U.S. Geological Survey OpenFile Report FL 68005, 241 p.

Stewart, J.W., Goetz, C.L., and Mills, L.R., 1978, Hydrogeologic factors affecting the availability and quality of ground water in the Temple Terrace area, Hillsborough County, Florida: U.S. Geological Survey Water-Resources Investigations Report 78-4, 38 p.

Stewart, J.W., and Mills, L.R., 1984, Hydrogeology of the Sulphur Springs area, Tampa, Florida: U.S. Geological Survey Water-Resources Investigations Report 83-4085, 1 sheet.

Tibbals, C.H., 1975, Aquifer tests in the summit reach of the proposed Cross-Florida Barge Canal near Ocala, Florida: U.S. Geological Survey WaterResources Investigations 28-75, $42 \mathrm{p}$.
Tibbals, C.H., Anderson, Warren, and Laughlin, C.P., 1980, Ground-water hydrology of the Dade City area, Pasco County, Florida, with emphasis on the hydrologic effects of pumping from the Floridan aquifer: U.S. Geological Survey WaterResources Investigations Report 80-33, 64 p.

Todd, D.K., 1980, Ground water hydrology: New York, John Wiley, 336 p.

Wetterhall, W.S., 1964, Geohydrologic reconnaissance of Pasco and southern Hernando Counties, Florida: Florida Geological Survey Report of Investigations 34,28 p.

Wolansky, R.M., and Corral, M.A., Jr., 1985, Aquifer tests in west-central Florida, 1952-76: U.S. Geological Survey Water-Resources Investigations Report 84-4044, 127 p.

Wolansky, R.M., Spechler, R.K., and Buono, Anthony, 1979, Generalized thickness of the surficial deposits above the confining bed overlying the Floridan aquifer, Southwest Florida Water Management District: U.S. Geological Survey Water-Resources Investigations/Open-File Report 79-1071, 1 sheet.

Yobbi, D.K., Mills, L.R., and Woodham, W.M., 1980, Ground-water levels in selected well fields and in west-central Florida, May 1980: U.S. Geological Survey Open-File Report 80-1001, 2 sheets.

Yobbi, D.K., and Woodham, W.M., 1981, Groundwater levels in selected well fields and in westcentral Florida, May 1981: U.S. Geological Survey Open-File Report 81-1106, 2 sheets. 